\title{
Structure-function-dynamics relationships in the peculiar Planktothrix PCC7805 OCP1: impact of his-tagging and carotenoid type.
}

Adjélé Wilson ${ }^{1 \S}$, Elena A. Andreeva ${ }^{2,3 \S}$, Stanislaw J. Nizinski ${ }^{4,5 \S}$, Léa Talbot ${ }^{1}$, Elisabeth Hartmann $^{3}$, IIme Schlichting ${ }^{3}$, Gotard Burdzinski ${ }^{5}$, Michel Sliwa ${ }^{4^{*}}$, Diana Kirilovsky ${ }^{1^{*}}$ and Jacques-Philippe Colletier ${ }^{2^{*}}$

${ }^{1}$ Université Paris-Saclay, CEA, CNRS, Institute for Integrative Biology of the Cell (I2BC), 91198 Gif-sur-Yvette, France.

${ }^{2}$ Univ. Grenoble Alpes, CEA, CNRS, Institut de Biologie Structurale, 38000 Grenoble, France.

${ }^{3}$ Max-Planck-Institut für medizinische Forschung, Jahnstrasse 29, 69120 Heidelberg, Germany.

${ }^{4}$ Univ. Lille, CNRS, UMR 8516, LASIRE, LAboratoire de Spectroscopie pour les Interactions, la Réactivité et l'Environnement, Lille 59000, France

${ }^{5}$ Quantum Electronics Laboratory, Faculty of Physics, Adam Mickiewicz University in Poznań, Uniwersytetu Poznańskiego 2, Poznan 61-614, Poland

$\S$ These authors contributed equally to this work.

* Correspondence to: J.-P. Colletier (colletier@ibs.fr), D. Kirilovsky (diana.kirilovsky@cea.fr); M. Sliwa (michel.sliwa@univ-lille.fr)

Keywords: cyanobacteria - flash photolysis - photosynthetic pigments - structure function relationships - X-ray diffraction

\section{Highlights:}

- Complete functional characterization of Synechocystis and Planktothrix OCPs

- Hitherto unknown structures of ECN- and CAN-functionalized Planktothrix OCP

- Insights into fs-s timescale photodynamics of ECN- and CAN-functionalized Synechocystis and Planktothrix OCPs 


\begin{abstract}
The orange carotenoid protein (OCP) is a photoactive protein involved in cyanobacterial photoprotection. Here, we report on the functional, spectral and structural characteristics of the peculiar Planktothrix PCC7805 OCP (Plankto-OCP). We show that this OCP variant is characterized by higher photoactivation and recovery rates, and a stronger energy-quenching activity, compared to other OCPs studied thus far. We characterize the effect of the functionalizing carotenoid and of his-tagging on these reactions, and the time scales on which these modifications affect photoactivation. The presence of a His-tag at the C-terminus has a large influence on photoactivation, thermal recovery and PBS-fluorescence quenching, and likewise for the nature of the carotenoid that additionally affects the yield and characteristics of excited states and the ns-s dynamics of photoactivated OCP. By solving the structures of Plankto-OCP in the ECN- and CAN-functionalized states, each in two closely-related crystal forms, we further unveil the molecular breathing motions that animate Plankto-OCP at the monomer and dimer levels. We finally discuss the structural changes that could explain the peculiar properties of Plankto-OCP.
\end{abstract}




\section{Introduction}

Photosynthetic organisms have evolved to make use of nearly all photons absorbed by their light-harvesting antennas. Under light stress conditions, however, the photosynthetic electron transport chain becomes saturated leading to the formation of harmful reactive oxygen species (ROS), e.g., singlet oxygen $\left({ }^{1} \mathrm{O}_{2}\right)$ [1, 2], that can damage the photosystems and other cellular machineries, eventually leading to cell death. Accordingly, photosynthetic organisms have developed a variety of mechanisms, altogether referred-to as non-photochemical quenching (NPQ), that are aimed at reducing the amount of energy reaching the photochemical reactions centers thereby avoiding accumulation of ROS [3]. In a vast majority of cyanobacterial strains, the main light-harvesting antenna is a large soluble complex, the phycobilisome (PBS), and the soluble $35 \mathrm{kDa}$ photoactive Orange Carotenoid Protein (OCP) is at the center of the NPQ mechanism (for review: [4-6]). OCP is capable both of dissipating the excess energy harvested by the PBS [7], and of quenching the produced harmful singletoxygen [8, 9]. For the energy quenching mechanism to be elicited, OCP must be photoactivated, which triggers the changes in protein structure and pigment position required for PBS binding and discharge of its excessive energy [10, 11]. Specifically, upon absorption of a blue-green photon, OCP converts from an inactive dark-adapted state (denoted as $\mathrm{OCP}^{\mathrm{O}}$, due to its orange color) into an active light-adapted state (denoted $O C P^{R}$, due to its red color). $\mathrm{OCP}^{\circ}$ is characterized by two absorption maxima at 475 and $495 \mathrm{~nm}$ (vibronic structure), while $O C P^{R}$ displays a single broader absorption peak between 510 and $530 \mathrm{~nm}$ [11]. The photoactivation quantum yield of the protein is notoriously low, viz. $0.2 \%$ [12], meaning that the OCP-supported photoprotective mechanism is at play only under high light conditions and that the concentration of $O C P^{R}$ is null, or very low, in darkness and under low light conditions $[11,13]$. Phylogenic studies of OCP sequences allowed their classification into three distinct clades, viz. OCP1, OCP2 and OCPX [14]. Members of the OCP1 clade are characterized by a slow $O C P^{R}$ to $O C P^{O}$ thermal recovery $\left(\right.$ at $\left.8^{\circ} \mathrm{C}\right)$ that is accelerated by the presence of the fluorescence recovery protein (FRP), whereas OCP2 and OCPX exhibit a faster thermal recovery (even at $8^{\circ} \mathrm{C}$ ) that is not affected by the presence of $\operatorname{FRP}[15,16]$.

The best characterized OCPs are OCP1s from Arthrospira maxima and Synechocystis PCC 6803, hereafter referred to as Arthrospira and Synechocystis OCP, respectively. For both, the dark-adapted structure was solved [8, 17], which revealed a conserved two-domain modular architecture. The fully $\alpha$-helical N-terminal domain (NTD, residues 1-165), unique to cyanobacteria, and the C-terminal domain (CTD, residues 187-320), structurally belonging to the nuclear transport factor-2 superfamily (NTF2), encase at their interface a ketocarotenoid pigment, e.g. 3'-hydroxyechineone (3'-hECN) $[8,17]$. Notwithstanding the presence of a linker that covalently attaches the NTD and CTD, the dark-adapted state is stabilized by two main 
protein interfaces, viz. (i) the central interdomain interface, which features two highlyconserved H-bond (N104-W277) and salt-bridge (R155-E244); and (ii) the interface between the $\mathrm{N}$-terminal helix (also coined, N-terminal extension or NTE) and the CTD $\beta$-sheet [8], which features six to seven $\mathrm{H}$-bonds depending on species. Additionally, the ketocarotenoid pigment buries $\approx 95 \%$ of its highly hydrophobic surface into the binding tunnel spanning the two domains, and therefore also contributes to the stabilization of the $\mathrm{OCP}^{\circ}$, with a buried surface area (BSA) of $786 \AA^{2}$ and a surface complementarity of $\sim 83 \%$. The sole polar interactions between the ketocarotenoid and the protein scaffold are the $\mathrm{H}$-bonds established between the carbonyl oxygen of its $\beta 1$ ring and the side chain hydroxyl and amine of CTD residues Y201 and W288 (Synechocystis OCP residue numbering), respectively $[8,17]$. Rupture of these $\mathrm{H}$ bonds is the first event along a photo-activation cascade that involves several 'red' intermediate states spanning the ps to second time scale [12, 18-20] and culminates with dissociation of the two domains following the $12 \AA$ translocation of the carotenoid into the NTD [21]. Dissociation of the two domains is essential for the energy-quenching function, as only $O C P^{R}$ is capable of binding to the PBS $[10,11]$. This activity is measured as the quenching of PBS fluorescence, itself induced by exposure to blue-light.

Despite the considerable knowledge acquired on OCP in the last decades, the photoactivation mechanism is still under debate. Notably, the very first instants of the photoactivation mechanism remains elusive. Numerous computational and time-resolved spectroscopic studies have recently sought to shed light on the structure, formation and decay of the carotenoid excited states associated with OCP photoactivation ([19, 22-24]; Figure 1). Notably, it was demonstrated that upon photoexcitation, three ps-lived intermediate states are formed following the sub-ps decay of the initial $S_{2}$ state, viz. an $S_{1}$ and an intra-molecular charge transfer (ICT) excited states $[25,26]$, and an $S^{*}$ state [19] that was initially proposed to correspond to a vibrationally hot $\mathrm{S}_{0}$ population [27]. Recently, however, it was proposed that the $S^{*}$ state, characterized by a longer lifetime than the $S_{1}$ and ICT states $[19,28]$, is also an excited state which serves as the precursor of the first photoproduct, $\mathrm{P}_{1}$, in which the $\mathrm{H}$-bonds between the carotenoid and the protein are broken and the protein is (therefore) already 'red' (difference absorption spectrum peaking at $565 \mathrm{~nm}$ ) [19]. The debate however remains open concerning the nature of the $S^{*}$ state [29-31] and its putative role as the precursor of $P_{1}$. Indeed, recent results from our laboratories show that while the photoactivation speed of $O C P^{R}$ is independent of irradiation light ( $470 \mathrm{~nm}$ versus $540 \mathrm{~nm}$ ), the concentration of $S^{*}$ decreases by $\approx 30 \%$ when $540 \mathrm{~nm}$ light is used to trigger photoactivation (Nizinski et al., submitted; doi.org/10.1101/2021.12.26.474187). Hence, $\mathrm{S}^{*}$ cannot be the sole precursor of $\mathrm{P}_{1}$. Evolution of this state (50 ns lifetime), wherein the carotenoid is likely untethered from its CTD H-bonding partners, leads to a repositioning of the carotenoid in the tunnel, in close vicinity of the dark- 
state position ( $\left.\mathrm{P}_{2} ; 0.5-1 \mu \mathrm{s}\right)$ [19]. After a first partial movement into the NTD (P2'; $\left.10 \mu \mathrm{s}\right)$ [19], the ketocarotenoid completes its translocation into the NTD, reaching the position it occupies in the final $O C P^{R}$ in around $10 \mu \mathrm{s}\left(\mathrm{P}_{3}\right) ;[19,23]$. Conformational changes in the NTE and CTT ensue ( $P_{M}$ and $P_{X} \sim 10 \mathrm{~ms}$ and $35 \mathrm{~ms}$ ), followed by an opening of the protein upon dissociation of the two domains ( $100 \mathrm{~ms})$ [23]. Thus, the formation of the photoactive $O C P^{R}$ is a multi-step reaction spanning at least fourteen decades in times $[12,19,23]$. All steps, excluding the $P_{2}$, to $\mathrm{P}_{3}$ and $\mathrm{P}_{3}$ to $\mathrm{P}_{\mathrm{M}}$ transitions, are accompanied by recovery to the initial $O C \mathrm{P}^{\circ}$ state, explaining the low quantum yield. About 1 and $0.2 \%$ of molecules reach the $P_{1}$ state [19] and the final $O C P^{R}$ state $[12,19,23]$ (Nizinski et al., submitted; doi.org/10.1101/2021.12.26.474187) (Figure 1), respectively.

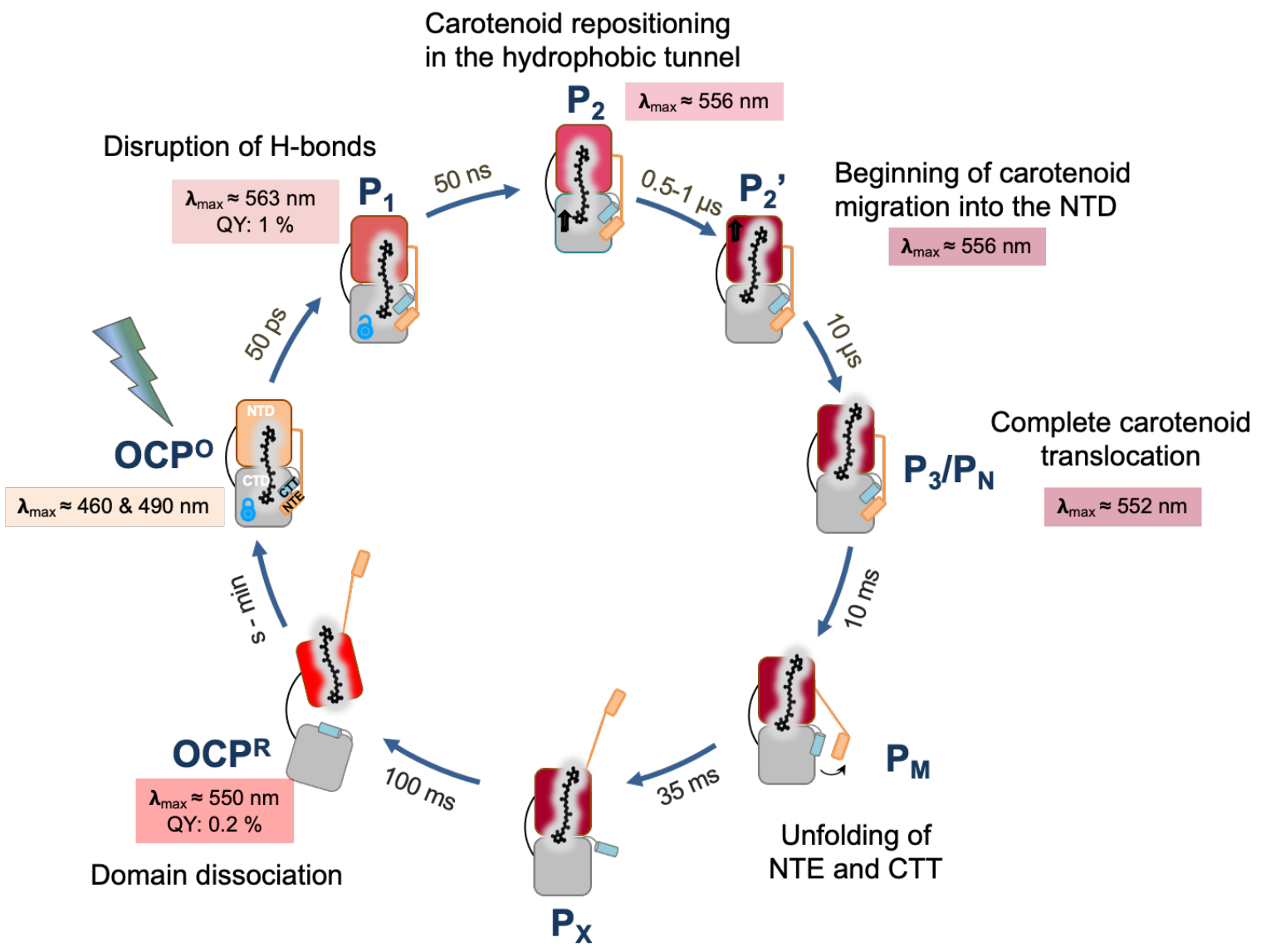

Figure 1: Formation of $O C P^{R}$ is a multi-step reaction that spans 12 decades in time. The figure which presents a working model for OCP photoactivation, summarizes findings from multiple studies $[12,19,23]$. The wavelength of maximum difference-absorbance (after subtraction of the OCP signal) is indicated for all intermediate states characterized to date. The $P_{1}, P_{2}, P_{2}$, and $P_{3}$ were observed by Konold et al. 2019 in transient absorption UV-Vis experiments upon excitation at $470 \mathrm{~nm}$ of Ntag-Syn-OCPECN [19]. Of important note, $\mathrm{P}_{2}$ ' was only observed in transient IR experiments, suggesting that it is characterized by a conformational change in the protein scaffold that does not influence the carotenoid electronic properties. $\mathrm{P}_{\mathrm{N}} / \mathrm{P}_{\mathrm{M}}$ and $\mathrm{Px}$ were observed by Maksimov et al. in time-resolved fluorimetry experiments upon excitation at $262 \mathrm{~nm}$ of the ECN-functionalized OCP-3FH mutant $(\mathrm{W} 41 \mathrm{~F}, \mathrm{~W} 101 \mathrm{~F}, \mathrm{~W} 110 \mathrm{~F}$ and $\mathrm{W} 277 \mathrm{H})$ featuring a single tryptophan at position W288 [23]. 
In all recent studies on OCP, the protein was expressed recombinantly [32] and to accelerate purification, a six-histidine tag (6XHis-tag) was introduced at the N-terminus (OCP produced in E. coli cells) or C- terminus (OCP produced in Synechocystis and E. coli cells). However, it was shown that after migration of the carotenoid from the NTD/CTD interface into the NTD $(\approx$ $0.5 \mu \mathrm{s})$ formation of $\mathrm{OCP}^{\mathrm{R}}$ requires, prior to domain separation, the detachment of the $\mathrm{N}$ terminal helix $\alpha A$ (or NTE) from the CTD $\beta$-sheet [19, 33, 34]. Likewise, the C-terminal helix $\alpha \mathrm{N}$ (or CTT) was proposed to reposition upon domain separation - possibly to "clog" the otherwise-exposed highly-hydrophobic CTD-half of the carotenoid tunnel [34-36]. Hence, presence of a His-tag at either the $\mathrm{N}$ - or C-terminal extremity of the protein can influence the kinetics of photoactivation and thermal recovery, respectively. In support of this hypothesis, we recently reported the finding that His-tag position affects: i) the photoactivation efficiency, with $O C P^{R}$ accumulation being slower when the His-tag is present at the $\mathrm{C}$-terminus (C-tagged OCP); ii) the lifetime of excited states, with those being shorter for C-tagged OCP); and iii) the spectral signatures of $S_{1}$ and $S^{*}$ states. However, the position of the tag does not influence P1 formation quantum yield (QY) suggesting that the more efficient photoactivation of the $\mathrm{N}$ tagged protein is related to molecular events occurring on the nano- to millisecond timescales (Nizinski et al., under revision). Regardless, it also remains unclear whether or not presence of the His-tag has an influence on PBS energy quenching and on thermal recovery. Also lacking is a thorough structure-function-dynamics comparison between OCP from various strains to understand how subtle changes in the structure may affect the function, its regulation or both. For example, it was recently reported that despite a lower intracellular OCP concentration and a quasi-strict conservation of the amino acids lining the OCP carotenoid tunnel, the amplitude of the OCP-related PBS-fluorescence quenching is larger in the cyanobacterium Planktothrix PCC 7805 than in Synechocystis PCC 6803 [37], and likewise for the PBS-fluorescence recovery. Hence, the Planktothrix OCP may differ in structure and/or structural dynamics from the other OCPs described to date.

Finally, it has been shown that while the naturally-occurring pigment in Synechocystis and Arthrospira OCP is 3'-hydroxyechinenone (3'-hECN), the protein may also be functionalized by other similarly-length ketocarotenoids, the palette of which will depend on species. Indeed, upon knock-out of the gene coding for the hydrolase converting ECN into hECN, Synechocystis OCP binds ECN [38], and likewise when the protein is overexpressed in Synechocystis cells, due to the low amount of hECN ( $\approx 1 \%$ of all ketocarotenoids) [11, 39]. When expressed recombinantly in E. coli cells [32], Synechocystis OCP can be complexed with ECN, canthaxanthin (CAN) or zeaxanthin (ZEA), depending on the set of carotenoid-producing genes that are co-transformed with the gene coding for the OCP. This feature holds true for all OCP variants produced recombinantly in carotenoid-producing E. coli cells, including OCP1 
from Synechocystis, Arthrospira and Tolypothrix [15, 32], OCP2 from Tolypothrix and Synechocystis 7508 [15, 16], and OCPX from Scytonema and Synechocystis 7508 [16]. To date, only Tolypothrix OCP was found to bind canthaxanthin (CAN) when overexpressed in Tolypothrix cells [30]. Early studies showed that the relative populations of the carotenoid $\mathrm{S}_{1}$ and ICT excited-states depend on the carotenoid that functionalizes OCP, with virtually no ICT in Synechocystis OCP functionalized with the fully symmetric CAN, but up to $50 \%$ ICT in OCP functionalized with the non-symmetric $\operatorname{ECN}[30,40]$. It was then proposed that the absence of the ICT state is a consequence of the fully symmetric nature of the CAN pigment, which is absent in $\operatorname{ECN}[30,40]$. However, the $\mathrm{P}_{1}$ state was not identified at the time, so that it remains elusive whether or not the change in functionalizing carotenoid also affects the yield of this presumed photoproductive intermediate. Moreover, it is unknown if the rate of OCPR accumulation and recovery as well as the yield of intermediary photoproducts are influenced by the nature of the functionalizing carotenoid.

Here, we address these gaps in knowledge by performing a comparative structure-function study on the OCP1 from two different strains, viz. Synechocystis PCC 6803 (Syn-OCP), and Planktothrix PCC 7805 (Plankto-OCP). For both OCP1 variants, the kinetics of photoactivation, thermal recovery and PBS-fluorescence quenching were assessed in the native (non-tagged), $\mathrm{N}$-tagged and $\mathrm{C}$-tagged states, and with ECN or CAN as the functionalizing carotenoid. We observe that the presence of the His-tag at the C-terminus has a larger influence on photoactivation, thermal recovery and PBS-fluorescence quenching than its presence on the $\mathrm{N}$-terminus. We found that the nature of the carotenoid influences the yield and characteristics of excited states, the ns-s dynamics of photoactivated OCP and the thermal recovery, leading to different rates of $O C P^{R}$ accumulation, and of PBS-fluorescence quenching. As only the structures of ECN- and CAN-functionalized Syn-OCP [17, 21] were available, we solved the Plankto-OCP structures in both the ECN- and CAN-functionalized states. At $1.4-1.8 \AA$ resolution, our structures shed light on the molecular breathing motions that animate PlanktoOCP monomers and dimers, and point to subtle changes outside of the carotenoid tunnel explaining the peculiar properties of Plankto-OCP. 


\section{Materials and Methods}

\section{Construction of plasmids for ocp gene expression in E. coli cells.}

The plasmids pCDF-OCPSynCtag (coding for C-tagged Syn-OCP), pCDF-NtagOCPSyn (coding for N-tagged Syn-OCP) featuring the Synechocystis ocp gene were described in [32]. To construct the plasmid pCDF-OCPSyn, and thereafter express the native (non-tagged) Synechocystis ocp (srl963) gene in E. coli cells, the nucleotides coding for the N-terminal histag in the plasmid pCDF-NtagOCPSyn were excised by mutagenesis using F-ocpSynNative: 5'-ATAAGGAGATATACCATGCCATTCACCATTGACTCT-3' and R-Duet: 5'CATGGTATATCTCCTTATTAAAGTTAAACAAAATTA-3' primers in order to create the plasmid $p C D F-O C P S y n$.

To construct the plasmid containing the Planktothrix agardhii PCC 7805 ocp (PLAM_2315) gene, a PCR using genomic DNA of Planktothrix agardhii str. 7805 as template and forward (F-OCPPlank EcoR1: 5'-CGATGCGAATTCTTCATTTACAGTCGATTCAGCCC-3') and reverse (R-OCPPlank Not1: 5'-CATTATGCGGCCGCCTTCCCCCTTAAATCACAAG-3') primers was performed. Then, the PCR products were cloned in pCDFDuet-1 previously digested by EcoRI and Notl which includes the nucleotides encoding for $6 x$ His-terminal tag. This plasmid, named pCDF-NtagOCPPx, was thereafter used to express Planktothrix OCP his-tagged at the $\mathrm{N}$-terminus.

To synthesize Planktothrix OCPs without tags, the nucleotides coding the 6x His-tag in the pCDF-NtagOCPPx plasmid were suppressed using F-OCPPxNoTag: 5'TTAATAAGGAGATATACCATGTCATTTACAGTCGATTCAGCCCGTGGG-3' and R-Duet (see above) to create the plasmid pCDF-OCPPx and express the native (non-tagged) Planktothrix OCP. Then, a nucleotide sequence encoding 6 histidine residues was added at the 3 'end by mutagenesis using the plasmid pCDF-OCPPx without tag as template and synthetic primers (F-DuetOCPPx 5'CACCACCACCACCACCACTAATTAATAAACGAATCTAATTTGATATAGC-3' and RDuetOCPPX Ctag: 5'GTGGTGGTGGTGGTGGTGACGAACTAAATTTAACAACTCTTTAGGTG-3'). Thereby, we obtained the plasmid pCDF-CtagOCPPx which was used to express Planktothrix OCP histagged at the $\mathrm{C}$-terminus.

\section{Construction of plasmids for frp gene expression in E. coli cells.}

The plasmid used to express the Synechocystis frp gene in E. coli was described in [10]. To express the Planktothrix agardhii PCC 7805 frp (PLAM_2314) gene in E. coli, the frp gene was amplified by PCR using forward (F-FRPPlank EcoR1: 5'CGATGCGAATTCTCAAGTAAATGAGATTGAATG-3') and reverse (R-FRPPlank Not1: 5'- 
TGCTTAGCGGCCGCAACTCAAATTGTTTTAAGAATCCCCG-3') primers. The resulting fragment was cloned between the EcoRI and Notl sites of the pCDFDuet-1 plasmid which contains the nucleotides encoding for $6 x$ His-tag.

\section{Holo-OCPs production, Isolation and Purification}

The production and isolation of holo-OCPs were previously described [32]. FRP isolation was described in [10]. To isolate OCPs without tag, E. coli cells were resuspended in lysis buffer (40 mM Tris-HCl pH 8, 1 mM EDTA, 1 mM PMSF, 1 mM caproic acid, 1 mM benzamidic acid, $50 \mu \mathrm{g} \mathrm{mL}^{-1}$ DNAse) and broken using a French Press. Membranes were pelleted and the supernatant was loaded on a Whatman DE-52 cellulose column. The OCP was eluted using a gradient of $60-80 \mathrm{mM} \mathrm{NaCl}$ in $40 \mathrm{mM}$ Tris- $\mathrm{HCl} \mathrm{pH}$ 8. A second purification step was performed by hydrophobic interaction chromatography (HiTrap Phenyl HP column, GE Healthcare) and the OCP was eluted in $40 \mathrm{mM}$ Tris- $\mathrm{HCl} \mathrm{pH} 8,0.5 \mathrm{M} \mathrm{NaCl}$. The eluate was dialyzed overnight against $40 \mathrm{mM}$ Tris- $\mathrm{HCl} \mathrm{pH} 8$ at $4^{\circ} \mathrm{C}$ (2 Liters).

\section{Absorbance measurements and kinetic analysis.}

Absorbance spectra of PBS and OCPs and kinetics of OCP photoactivation (illumination with $5000 \mu \mathrm{mol}$ photons. $\mathrm{m}^{2}$. $\mathrm{s}^{-1}$ of white light), and dark recovery were measured with a Specord S600 spectrophotometer (Analytic Jena) using $1 \mathrm{~cm}$ path-length cuvette. Experiments were performed at an OCP concentration of $\sim 4.8 \mu \mathrm{M}$, corresponding to an OD of 0.3 at $496 \mathrm{~nm}$ (calculated using an epsilon equal to $63000 \mathrm{M}^{-1} \cdot \mathrm{cm}^{-1}$ [41]). Spectra were acquired in the 250$700 \mathrm{~nm}$ range for each time point. $\mathrm{OCP}^{\mathrm{O}}$ photoactivation and $O C \mathrm{P}^{\mathrm{R}}$ recovery were both monitored by measuring the changes of absorbance at $550 \mathrm{~nm}$.

\section{Isolation of PBS and fluorescence measurements.}

The purification of PBS from Synechocystis PCC 6803 and Planktothrix agardhii PCC 7805 was performed as previously described [42]. The PBS-fluorescence quenching yield was monitored using a pulse amplitude-modulated fluorimeter (101/102/103-PAM, Walz). Measurements were made in a $1 \mathrm{~cm}$ path-length stirred cuvette. PBS quenching induced by holo-OCPs was measured in 0.5 potassium phosphate buffer $(\mathrm{pH} 7.5)$ at $23^{\circ} \mathrm{C}$ under strong blue-green light $\left(900 \mu \mathrm{mol}\right.$ photons $\left.\mathrm{m}^{-2} \mathrm{~s}^{-1}\right)$. The PBS concentration used was $0.012 \mu \mathrm{M}$ and the ratio of OCP to PBS was 40:1. OCP samples were pre-illuminated with $5000 \mu \mathrm{mol}$ photons $\mathrm{m}^{-2} \mathrm{~s}^{-1}$ of white light at $4^{\circ} \mathrm{C}$. Samples in Pasteur pipettes were quickly frozen by immersion in liquid nitrogen. Fluorescence emission spectra at $77 \mathrm{~K}$ were recorded using a CARY Eclipse fluorescence spectrophotometer fluorometer (Varian). 


\section{Protein Separation}

Proteins were analyzed by SDS-PAGE on 15\% polyacrylamide gels in a Tris-MES buffer [43]. PBS samples were concentrated by precipitation with $10 \%(\mathrm{v} / \mathrm{v})$ TCA prior to loading (equal protein quantities in each lane). $10 \mu \mathrm{L}$ at a PBS concentration of $0.5 \mu \mathrm{M}$ were deposited per well. The gels were stained by Coomassie Brilliant Blue.

\section{Femtosecond transient absorption spectroscopy (0 - 1 ns timescale)}

Transient absorption measurements were performed using a Helios system from Ultrafast Systems. A short-pulse titanium sapphire oscillator (Mai Tai, Spectra Physics, 70 fs pulse length) followed by a high-energy titanium sapphire regenerative amplifier (Spitfire Ace, Spectra Physics, $100 \mathrm{fs}, 1 \mathrm{kHz}$ ) provided the $800 \mathrm{~nm}$ beam, which was further split to generate: (1) a $532 \mathrm{~nm}$ pump pulse in the optical parametric amplifier (Topas Prime with a NirUVVis frequency mixer) and (2) a white light continuum probe pulse in a sapphire crystal (430-780 $\mathrm{nm})$. The remaining $800 \mathrm{~nm}$ probe pulse photons were filtered just after white light continuum generation. The instrument response function (IRF) was estimated to be around $110 \mathrm{fs}$ (FWHM). The pump diameter (FWHM) at the sample was approximately $250 \mu \mathrm{m}$, for a pulse energy of $0.8 \mu \mathrm{J}$. Absorbance was close to 0.7 at the excitation wavelength over a $2 \mathrm{~mm}$ optical path (55 $\mu \mathrm{M}$ or $1.9 \mathrm{mg} / \mathrm{mL}$ ). The sample solution was stirred to keep the OCP solution fresh in the probed volume. The pump beam was depolarized to avoid anisotropy effects. To ensure that datasets are comparable to each other, they were all measured under identical conditions $\left(\right.$ at $22^{\circ} \mathrm{C}$ ) during a single experimental session. The transient absorption data were corrected for the chirp of white light continuum by aligning kinetics according to a delay introduced by given amount of the material between the OCP sample and the sapphire crystal (used for WLC generation of the probe). For all datasets, the difference absorbance $(\Delta \mathrm{A})$ value obtained at the bleaching extremum (in both spectral and temporal dimension) was normalized to -1 . Transient spectra were projected onto a $5 \mathrm{~nm}$-spaced grid to get kinetic traces. The comparison of pre-exponential factors at $490 \mathrm{~nm}$ (bleaching band) allowed us to estimate the quantum yield of the formation of the various intermediates. We used for interpretation the most recent proposed model (Nizinski et al., submitted; doi.org/10.1101/2021.12.26.474187), whereby $S_{1}, I C T$ and $S^{*}$ are formed from $S_{2}$ in parallel paths and decay mainly to $S_{0}$ without any interconversion and with only small contribution of excited-state absorption at $490 \mathrm{~nm}$. For $\mathrm{P}_{1}$ formation quantum yield, the positive absorbance contribution was taken into account (Nizinski et al., submitted; doi.org/10.1101/2021.12.26.474187). Time constants determination and calculation of Decay Associated Difference Spectra (DADS or DAS) were achieved by global analysis using our custom-Python package (Nizinski et al., submitted; doi.org/10.1101/2021.12.26.474187) and the Glotaran software (https://glotaran.org). For the global analyses, a sum of four exponentials $\left(S_{2}, I C T, S_{1}, S^{*}\right)$ convolved by a Gaussian IRF 
(fixed to $110 \mathrm{fs}$ ) and an offset (representing long-lived photo-products with lifetime $>50 \mathrm{~ns}$, namely $\mathrm{P}_{1}$ ) was used.

\section{Nanosecond transient absorption spectroscopy (50 ns - $1 \mathrm{~s}$ timescale)}

Measurements were performed with our custom apparatus described previously [44]. $532 \mathrm{~nm}$ nanosecond excitation pump pulses of $5 \mathrm{~mJ}$ energy were used (one pulse every $20 \mathrm{~s}$ for 100 ms kinetics, one pulse every $40 \mathrm{~s}$ for kinetics over $100 \mathrm{~ms}$ ). The probe light from the Xenon lamp was filtered using a $550 \mathrm{~nm}$ interference filter (10 nm FWHM) placed before the sample. Scattered pump light was removed by a notch filter set after the sample. For each experiment, a solution of OCP with absorbance close to 0.7 at excitation wavelength $(1 \mathrm{~cm}$ path-length, 11 $\mu \mathrm{M}$ or $0.39 \mathrm{mg} / \mathrm{mL}$ ) was placed in a $10 \times 10 \mathrm{~mm}$ cuvette and thermalized at $22^{\circ} \mathrm{C}$. No stirring of the sample was applied, enabling to stretch the time window covered by the experiments. Each set of measurements included 100 replicates of each of the five time-windows together covering the 50 ns - 1 s time range. Recorded data were merged and projected onto a logarithmic grid. Stability of the protein was checked by its steady-state absorbance after each experiment. The formation quantum yield of $O C P^{R}$ was determined using ruthenium as actinometer [45]. The difference molar absorption coefficient at $550 \mathrm{~nm}$ for $O C P^{R}$ was estimated using the molar absorption coefficient $(\varepsilon)$ of $O C P^{\circ}$ at $\left.490 \mathrm{~nm}=63000 \mathrm{~cm}^{-1} \cdot \mathrm{M}^{-1}\right)[41]$ and that determined for $O C P^{R}$ after $100 \%$ photo-conversion at $8^{\circ} \mathrm{C}\left(\varepsilon_{550} \mathrm{~nm} \approx 48000,41000\right.$, 47000 and $40000 \mathrm{~cm}^{-1} \cdot \mathrm{M}^{-1}$ for Syn-OCP ${ }_{\text {CAN }}$, Syn-OCP ${ }_{\text {ECN }}$, Plankto-OCP ${ }_{\text {CAN }}$ and Plankto$O C P^{R}{ }_{E C N}$, respectively). For each sample, data were fit globally over the five time- windows using a three-exponential model accounting for three different intermediates states and an offset, attributed to $\mathrm{OCP}^{\mathrm{R}}$, and therefore used to estimate the yield. Fitted results are shown in Table 1.

\section{Crystallization}

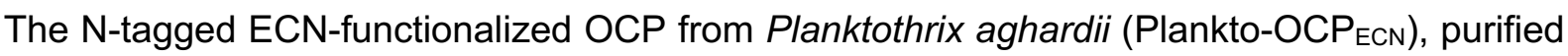
on Ni-NTA and phenyl-sepharose columns, was further subjected to size exclusion chromatography under dim red light (HiLoad 16/600 Superdex 75 pg, GE Healthcare). The protein eluted as a unique peak in $50 \mathrm{mM}$ Tris- $\mathrm{HCl}$ buffer $\mathrm{pH} 7.4,150 \mathrm{mM} \mathrm{NaCl}$. Crystallization conditions were screened manually, using as starting conditions those that afforded crystallization of Syn-OCP [17] and Arthrospira maxima OCP [8]. The gel-filtrated Plankto$\mathrm{OCP}_{\mathrm{ECN}}$ sample was concentrated to $5.2,6.6$ and $7.0 \mathrm{mg} / \mathrm{mL}$ (the protein concentration was determined based on the absorption at $495 \mathrm{~nm}$, using an extinction coefficient of $63000 \mathrm{M}^{-1}$ $\left.\mathrm{cm}^{-1}[41]\right)$ and crystallization trials were performed in 24-well Limbro plates using the vapor diffusion method in the hanging-drop geometry. Crystallization drops were set at $4^{\circ} \mathrm{C}$ by mixing $1 \mu \mathrm{L}$ from the well solution with $1 \mu \mathrm{L}$ of protein solution. The well solution, of $1 \mathrm{~mL}$ volume, was 
composed of $0.1 \mathrm{M}$ or $0.2 \mathrm{M}$ Bis-Tris pH 5.5 and increasing PEG 3550 concentrations (from $18 \%$ to $25 \%$ ) were tested. Crystals appeared within 3 to 5 months in $0.2 \mathrm{M}$ Bis-Tris pH 5.5, 20\%-25\% PEG 3550. Following this success, crystallization trials were optimized enabling growth of crystals in a few hours to few days at room-temperature $\left(\sim 20^{\circ} \mathrm{C}\right)$. Crystallization of Plankto-OCP $P_{\text {CAN }}$ was achieved using a protein concentration of $1.5-2 \mathrm{mg} / \mathrm{ml}$ in $50 \mathrm{mM}$ Tris$\mathrm{HCl} \mathrm{pH} \mathrm{7.4,} 150 \mathrm{mM} \mathrm{NaCl}$, a well solution composed of $0.1 \mathrm{M}$ sodium acetate, $\mathrm{pH} 5$ and 20$25 \%$ PEG4000, and by mixing these at 1:1 ratio in the crystallization drops.

\section{X-ray data collection and processing and structure determination}

$\mathrm{X}$-ray data were collected at $100 \mathrm{~K}$ from crystals cryoprotected by a short soak in the mother liquor complemented with 18-20 \% glycerol and directly frozen in the nitrogen gas stream at the European Synchrotron Radiation Facility (ESRF, Grenoble, France), at the Swiss Light Source (SLS, Villigen, Switzerland) or on the MicroMax-007 HF diffractometer (Rigaku) installed at the Max Planck Institute in Heidelberg (MPI-HD). Specifically, we used : (i) ESRF-

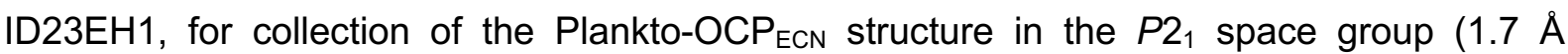
resolution; $\lambda=0.979 \AA$; beamsize: 30 (h) x 30 (v) $\mu \mathrm{m}^{2}$ ); (ii) ESRF-ID29, for collection of the Plankto-OCP $\mathrm{ECN}_{\text {in }}$ the $C 2$ space group (1.7 $\AA$ resolution; $\lambda=0.976 \AA$; beamsize: $30(\mathrm{~h}) \times 30(\mathrm{v})$ $\mu \mathrm{m}^{2}$ ); (iii) SLS-X10SA (PXII), for collection of the Plankto-OCP CAN structures in the $P 2{ }_{1}$ space group (1.4 resolution; $\lambda=0.99 \AA$; beamsize: 50 (h) $\times 10$ (v) $\mu \mathrm{m}^{2}$ ); and (iii) the MPI-HD diffractometer, for collection of the Plankto-OCP $P_{C A N}$ structures in the $P 2_{1}$ and C2 space group (1.85 Å resolution, respectively; $\lambda=0.99 \AA$; beamsize: $\left.50(\mathrm{~h}) \times 10(\mathrm{v}) \mu \mathrm{m}^{2}\right)$. Data were collected with an oscillation range of 0.1 (ID29 and ID23-EH1), 0.2 (SLS-X10SA (PXII) or 0.25 degree (MPI-HD). All data were indexed using XDS [46], and scaled and merged using XSCALE [47].

\section{Molecular replacement and structure refinement.}

Phaser [48] was used to phase by molecular replacement (MR) the data collected on crystalline Plankto-OCP $\mathrm{ECN}_{\mathrm{EN}}$ in the $\mathrm{C} 2$ space group, using as a starting model chain A from the Syn$\mathrm{OCP}_{\mathrm{ECN}}$ structure (PDB id: 3mg1, [17]). We used the CCP4 [49] buccaneer pipeline, based on Buccaneer [50], Parrot [51] and Refmac5 [52] for the initial in silico rebuilding of the C2 Plankto$\mathrm{OCP}_{\mathrm{ECN}}$ structure $(76.2 \%$ and $92.8 \%$ identity and similarity with respect to Syn-OCP), resulting in a model characterized by Rfree and Rwork values of 29.63 and 26.17 , respectively, and wherein 306 residues had been placed in sequence in two fragments corresponding to the NTD and CTD. Examination of this model revealed imperfections in loop building, which were corrected manually using the molecular graphics program Coot [53]. The C2 Plankto-OCP $\mathrm{ECN}_{\mathrm{EN}}$ structure was thereafter refined by iterative cycles of reciprocal-space refinement using Refmac5 and manual model-building in real-space using Coot. Therefrom, phasing of the C2 Plankto-OCP $P_{\text {CAN }}$ data was achieved by rigid-body refinement with Refmac5, while that of the 
$P 2{ }_{1}$ Plankto-OCP $\mathrm{ECN}_{\text {and }}$ Plankto-OCP $\mathrm{CAN}_{\mathrm{C}}$ structures was achieved by molecular replacement

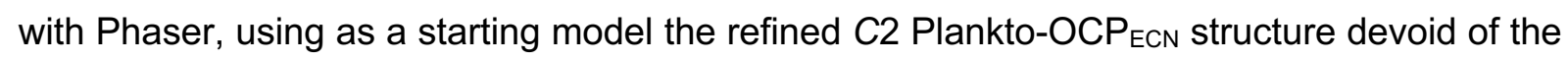
carotenoid, waters and protein alternate conformations. Refinement again consisted of iterative cycles of reciprocal-space refinement using Refmac5 and manual model-building in real-space using Coot. Using $\mathrm{C} 2$ Plankto-OCP $\mathrm{CAN}_{\mathrm{CA}}$ as the reference structure, Ca-Ca distance difference matrices were prepared using a custom-written script, and the hinge motions of domains within monomers and of monomers within dimers were evaluated using hinge_find.py script available at https://github.com/gawells/hingefind, inspired from the hinge_find.tcl script [54] available at http://biomachina.org/disseminate/hingefind/hingefind.html. The presence of tunnels in OCP, and notably the extent and volume of the carotenoid binding tunnel, was examined using Caver3 [55] and the dedicated PyMOL plugin available at https://pymolwiki.org/index.php/Caver3). Porcupine plots, showing for each structure the direction and distance travelled by $\mathrm{C} \alpha$ atoms with respect to the $\mathrm{C} 2$ Plankto-OCP $\mathrm{PAN}_{\text {structure, }}$ were prepared using the modevectors.py PyMOL script available at https://pymolwiki.org/index.php/Modevectors. Figures were prepared with PyMOL unless stated otherwise. Data processing and refinement statistics are shown in Table 2. Planktothrix OCP structures have been deposited in the wwPDB under accession codes 7qd0, 7qcZ, 7qd1 and $7 \mathrm{qd} 2$. 


\section{Results \\ Comparison of native (non-tagged) OCPs from Planktothrix aghardii and Synechocystis PCC6803}

The Synechocystis and Planktothrix species share the feature that only one ocp gene, classified in the OCP1 clade [16], and one frp gene are found in their genomes. The two OCP genes display $76.2 \%$ and $92.8 \%$ identity and similarity, respectively, with nearly all residues lining the carotenoid tunnel being conserved. We investigated whether or not the functionalizing carotenoid and his-tagging have an effect on the photoactivation and recovery kinetics of these two OCP, by expressing recombinantly the native (non-tagged), N-tagged and C-tagged Planktothrix and Synechocystis ocp genes in CAN or ECN producing E. coli cells (for details on constructs and on their expression and purification, see Materials and Methods section).

We first compared the spectral properties at $9.5{ }^{\circ} \mathrm{C}$ of the native (non-tagged) CANfunctionalized Syn-OCP and Plankto-OCP (Figure 2), viz. Syn-OCP CAN $_{\text {and Plankto-OCP }}$ CAN. The two proteins display identical absorption spectra in the dark-adapted (orange) inactive state $\left(\mathrm{OCP}^{\circ}\right)$, however slight differences are seen for the light-adapted (red) active state $\left(O C P^{R}\right.$ ) (Figures 2A, D and Supplementary Figure 1). Both $O C P^{R}{ }_{C A N}$ spectra present an absorption maximum at $530 \mathrm{~nm}$ with a shoulder at $560 \mathrm{~nm}$. This shoulder is slightly more pronounced in Syn-OCP ${ }^{R}$ CAN. (Figures $2 A$ and $2 D$ ). A difference positive absorbance maximum at $560 \mathrm{~nm}$ is observed in both OCPs (Figures $2 \mathrm{~B}$ and $2 \mathrm{E}$ ). The normalization of the difference spectra time series on the $470 \mathrm{~nm}$ peak not only confirms that the $\Delta \mathrm{A}(560 \mathrm{~nm})$ to $\Delta \mathrm{A}(470 \mathrm{~nm})$ ratio is higher in Syn-OCP ${ }^{R}$ CAN than in Plankto-OCP ${ }^{R}$ CAN (Figures $2 \mathrm{C}$ and $2 \mathrm{~F}$ ), but also evidences a red shift in the spectra of both $O C P^{R}$ (from 545 - 550 to $560 \mathrm{~nm}$ ) as they accumulate (Figures $2 \mathrm{C}$ and $2 \mathrm{~F}$ ).

We then assayed photoactivation and thermal recovery kinetics of Syn-OCP $\mathrm{CAN}_{\text {and Plankto- }}$ $\mathrm{OCP}_{\mathrm{CAN}}$ (Figure $2 \mathrm{G}$ and $\mathrm{H}$ ). Experiments were performed at $9.5^{\circ} \mathrm{C}$, to minimize the negative contribution of thermal recovery to the steady-state accumulation of $O C P^{R}-i$.e., to maximize $O C P^{R}$ concentration in the photostationary equilibrium. Accumulation of $O C P^{R}$ was monitored by following the rise in absorbance at $550 \mathrm{~nm}$ upon intense white-light illumination. Results in Figure 2 show that Plankto-OCPR ${ }_{\text {CAN }}$ not only accumulates significantly faster than Syn$O C P^{R}{ }_{C A N}$ (initial slope is twice as high), but it also recovers the dark $O C P^{O}$ state faster. Indeed, Plankto-OCP ${ }^{\mathrm{R}}$ CAN recovers fully within the 20 min lapse of our experiment (Figure $2 \mathrm{H}$ ), whereas only $30 \%$ of Syn-OCP ${ }^{R}$ has reconverted to $O C P^{O}$. The slow recovery kinetics at low temperature of Syn-OCPR ${ }_{\text {CAN }}$ is known, and shared by other OCP1s from Arthrospira and Tolypothrix $[15,16,42]$, whereas the fast recovery kinetics of Plankto-OCPR ${ }_{\text {CAN }}$ is 
unprecedented for members of the OCP1 clade. It is reminiscent of those displayed by members of the OCP2 $[14,16]$ and OCPX [16] clades. In these clades, the faster OCPR $\mathrm{OCP}^{\circ}$ recovery rate coincides with the inability to interact with $\operatorname{FRP}[15,16]$. Therefore, we challenged a possible misclassification of Plankto-OCP as a member of the OCP1 clade by investigating whether or not its recovery is accelerated by the presence of FRP. For this purpose, Synechocystis and Planktothrix FRPs were expressed and purified, and assayed for their species-specific effect on the CAN-functionalized native versions of OCP. Figure $2 \mathrm{I}$ shows that the presence of FRP accelerates the recovery rate of Plankto-OCP $\mathrm{CAN}_{\text {although the }}$ observed acceleration is smaller than for Syn-OCP CAN $_{\text {in }}$ presence of Syn-FRP. These results confirm the correct assignment of Plankto-OCP to the OCP1 clade. They also show that the species-specific acceleration by FRP of OCP recovery is independent of the rate of the reaction in the absence of FRP.

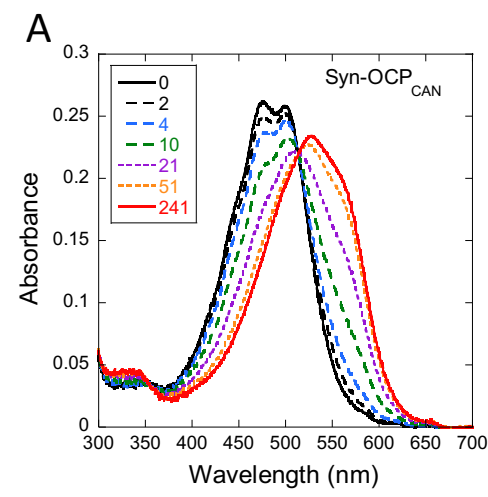

D

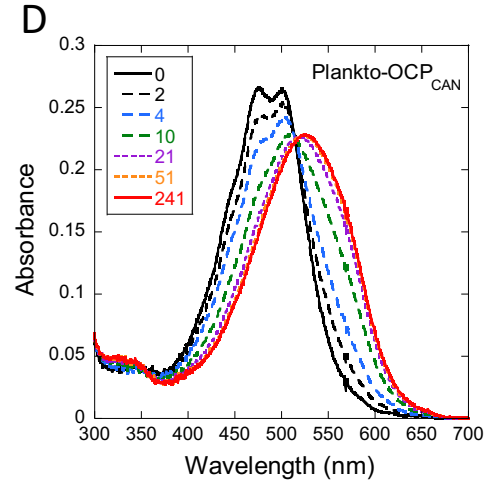

G

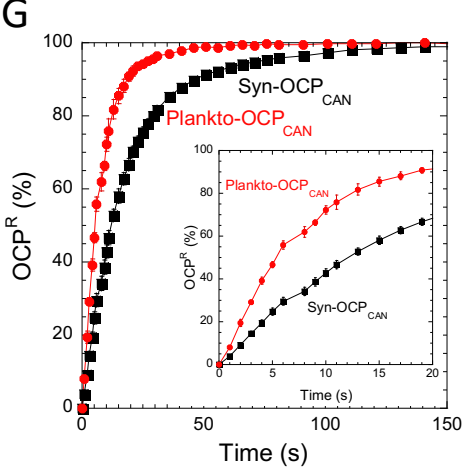

B

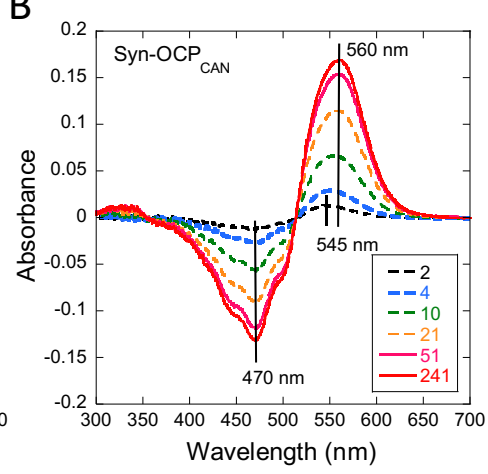

E

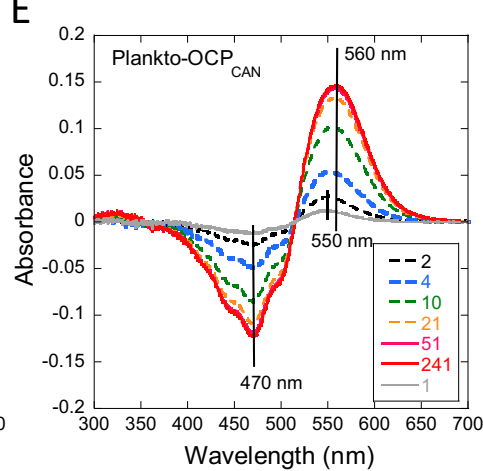

$\mathrm{H}$

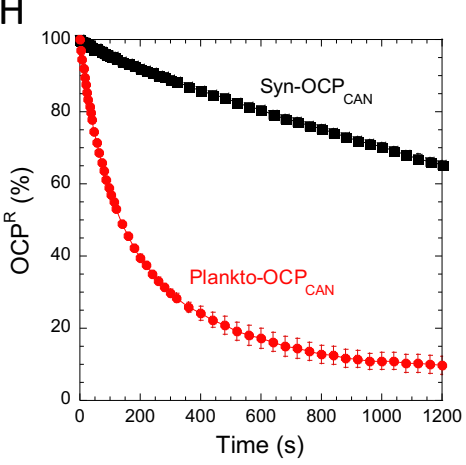

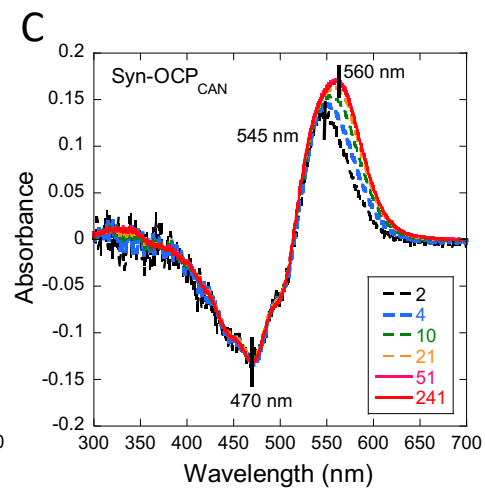

F

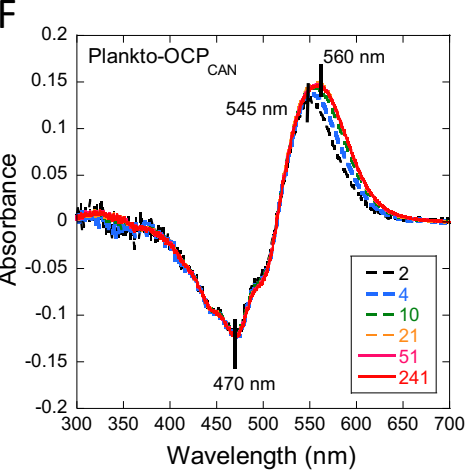

I

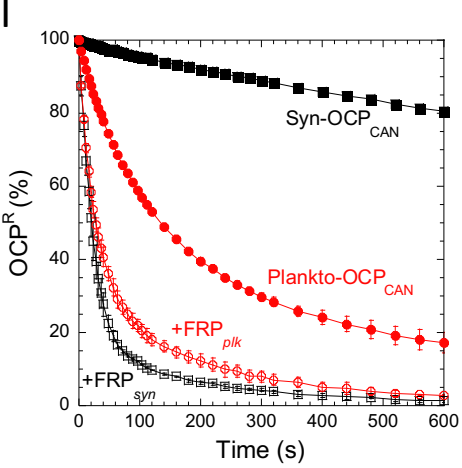


Figure 2: Photoactivation and recovery of native (non-tagged) CAN-functionalized Synechocystis and Planktothrix OCPs. (A and D) Absorbance spectra of Syn-OCPCAN (A) and Plankto-OCPCAN (D) at different times of illumination. (B and E) Differential absorbance spectra derived from $A$ and $D$ respectively. ( $C$ and $F$ ) Differential absorbance spectra normalized at $470 \mathrm{~nm}$ derived from $A$ and $D$. (G-I) Kinetics of photoactivation $(G)$ and recovery $(\mathrm{H}-\mathrm{I})$ of CAN-functionalized Syn-OCP (black) and Plankto-OCP (red). Inset in G: First $30 \mathrm{~s}$ of illumination. In (I), the effect of the presence of FRP is shown. The ratio FRP to OCP was 1:1. The accumulation of OCPR and its thermal deactivation were followed by increase and decrease of absorbance at $550 \mathrm{~nm}$. The OCPs were illuminated with white light $\left(5000 \mu \mathrm{mol}\right.$ photons $\left.\mathrm{m}^{-2} . \mathrm{s}^{-1}\right)$ at $9.5^{\circ} \mathrm{C}$. Error bars: standard deviation. Each curve represents the mean of three independent measurements, respectively.

\section{Influence of the His-tag on OCP photoactivation and recovery kinetics}

We have recently shown that the location of the His-tag influences the photoactivation speed (initial slope) in Synechocystis OCP (Nizinski et al., submitted; doi.org/10.1101/2021.12.26.474187). In particular, by using intermediary light intensity ( 100 $\mu \mathrm{mol}$ photons. $\mathrm{m}^{-2} . \mathrm{s}^{-1}$ ) we observed a more efficient accumulation of $\mathrm{OCP}^{\mathrm{R}}$ in Ntag-Syn$\mathrm{OCP}_{\mathrm{ECN}}$ than in Ctag-Syn-OCP $\mathrm{ECN}_{\mathrm{N}}$ despite a comparable $\mathrm{P} 1$ formation quantum yield. Hence, we here asked whether or not presence of a His-tag, and its location at the $\mathrm{N}$ - or C-terminus, would influence the photoactivation and thermal recovery of Plankto-OCP and Syn-OCP. We compared results obtained from the $\mathrm{N}$-tagged (Ntag-Syn-OCP $\mathrm{PAN}_{\text {an }}$ and Ntag-Plankto-OCP $\mathrm{PAN}_{\text {) }}$ and C-tagged (Ctag-Syn-OCP $P_{\text {CAN }}$ and Ctag-Plankto-OCP ${ }_{C A N}$ ) variants of these OCPs to those of the native counterparts. It was consistently observed that the $\mathrm{N}$-tagged OCPs photoactivate faster than their $\mathrm{C}$-tagged counterparts, with the native variants showing an intermediary behavior (Figure 3A and 3B). Nevertheless, when triggered with $5000 \mu \mathrm{mol}$ photons $\mathrm{m}^{-2} . \mathrm{s}^{-1}$ white light, the effect of tagging on photoactivation was not dramatic. Focusing next on the thermal $O C P^{R}$ to $O C P^{O}$ recovery, we found that it is delayed by presence of a His-tag in all tested OCPs (Figures $3 \mathrm{C}$ and $3 \mathrm{D}$ ), although the effect is clearly more visible for Plankto-

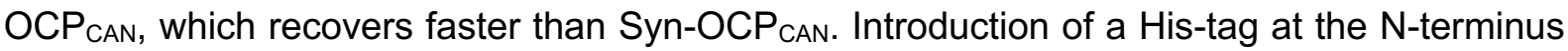
hardly effects the recovery of Plankto-OCP, whereas that at the C-terminus slows down the recovery by a factor of six (Figure 3D). In contrast, in Syn-OCP, His-tagging only has a slight effect on recovery.

\section{Influence of the functionalizing carotenoid on OCP photoactivation and recovery kinetics}

We then investigated the extent to which photoactivation and thermal recovery are affected by the type of functionalizing carotenoid. As it was found above that $\mathrm{N}$-tagged and native OCP are the most similar in terms of photoactivation and recovery rates, we used N-tagged SynOCP and Plankto-OCP in the following assays. Irrespective of the species, $\mathrm{OCP}^{\mathrm{R}}$ accumulation is slower in ECN-functionalized than CAN-functionalized OCP (Figures $4 A$ and 4B), while recovery is faster (Figure $4 C$ and $4 D$ ). For example, the initial slopes of $O C P^{R}$ accumulation 
by Ntag-Syn-OCP $\mathrm{CAN}_{\text {and Ntag-Plankto-OCP }}$ an are twice as high as those measured on their ECN-functionalized counterparts (Figures 4A and 4B). The most straightforward explanation for these observations is that CAN stabilizes $O C P^{R}$ and/or facilitate the carotenoid translocation during photoactivation. The red Syn-OCP $\mathrm{ECN}_{\mathrm{EN}}$ and Plankto-OCP $\mathrm{ECN}_{\mathrm{N}}$ are spectrally similar, both presenting a maximum absorption at $510 \mathrm{~nm}$ (Figures $4 \mathrm{E}$ and $\mathrm{H}$ ). In difference absorption spectra, the positive maximum is yet at $550.5 \mathrm{~nm}$, i.e., slightly blue shifted compared to the OCP ${ }_{\text {CAN }}$ counterparts (Figure 2 and Figures $4 \mathrm{~F}, \mathrm{I}$ ). It is notable that an increase in the $\Delta \mathrm{A}(550 \mathrm{~nm})$ to $\Delta \mathrm{A}(470 \mathrm{~nm})$ ratio is observed in the first 10 seconds of

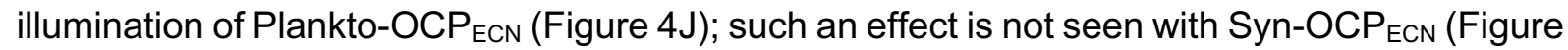
$4 G)$.

A

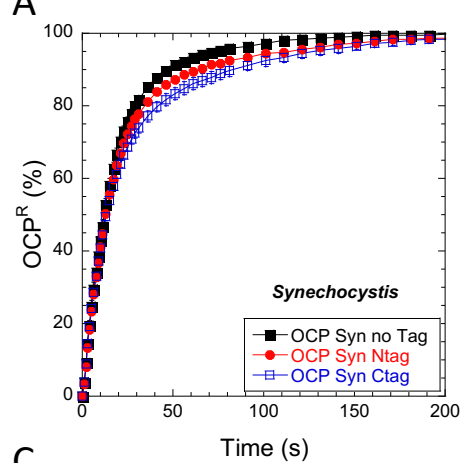

C

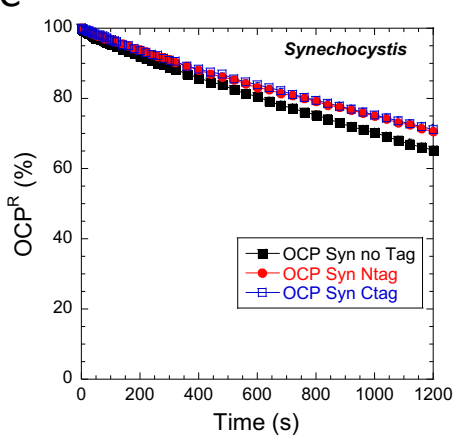

B
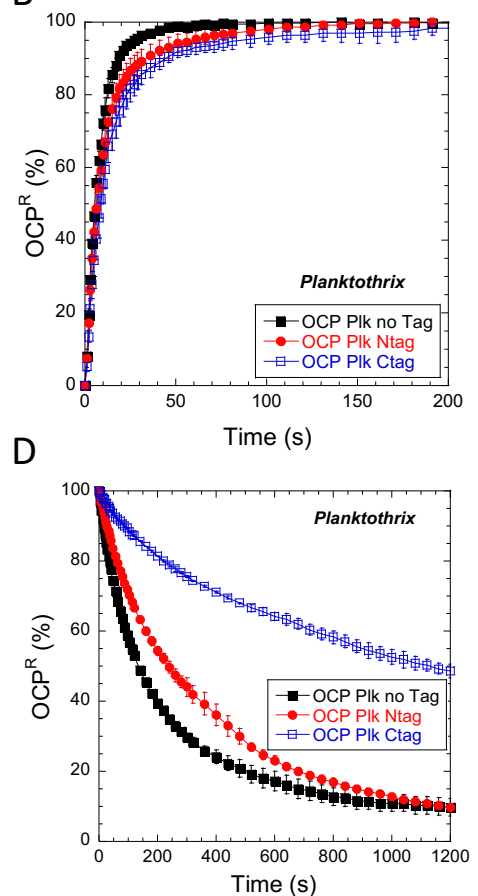

Figure 3: Presence of a Histag has an impact on photoactivation and recovery. Effect of presence and position of His-tag on photoactivation (A-B) and recovery (C-D) of CANfunctionalized Synechocystis and Planktothrix OCPs. The accumulation of $O C P^{R}$ and its thermal deactivation were followed by increase and decrease of absorbance at $550 \mathrm{~nm}$. The OCPs were illuminated with white light (5 $000 \mu \mathrm{mol}$ photons $\mathrm{m}^{-2} \cdot \mathrm{s}^{-1}$ ) at $9.5^{\circ} \mathrm{C}$. Error bars: standard deviation. Each curve represents the mean of three independent measurements.

By carrying out fs-ns timescale transient absorption spectroscopy on the four OCPs, we inquired whether or not the increased photoactivation speed of CAN-functionalized Planktoand Syn-OCP stem from changes in the carotenoid excited state dynamics. Thereby we could estimate the primary quantum yields for the formation of the five intermediates occurring during the initial $100 \mathrm{fs}-100$ ps of the photoactivation cascade, i.e., the $S_{2}, S_{1}$ and ICT excited-states, the $S^{*}$ state, and the first photoproduct $P_{1}$ (see Material and Methods section and Nizinski et al., submitted; doi.org/10.1101/2021.12.26.474187). Their formation and decay in CAN- and ECN-functionalized Plankto-OCP (Figure 5) and Syn-OCP (Supplementary Figure 2) were monitored by recording and globally-fitting femtosecond transient spectra collected at different 
time delays following a 110-fs pulse excitation at $532 \mathrm{~nm}$ (see the Materials and Methods section for further details).

A

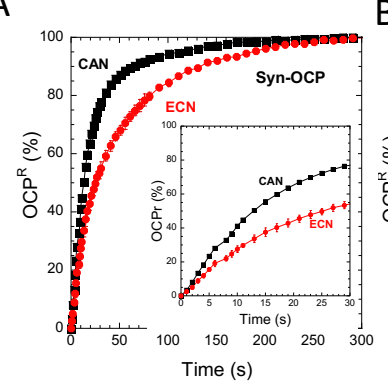

$\mathrm{E}$

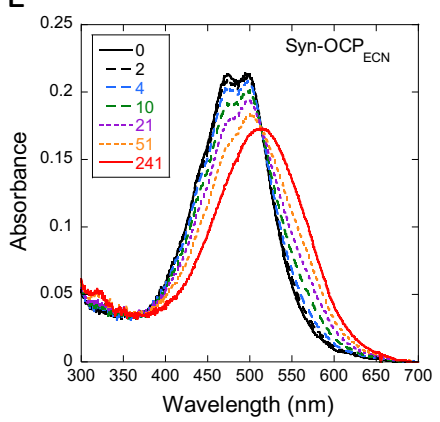

$\mathrm{H}$

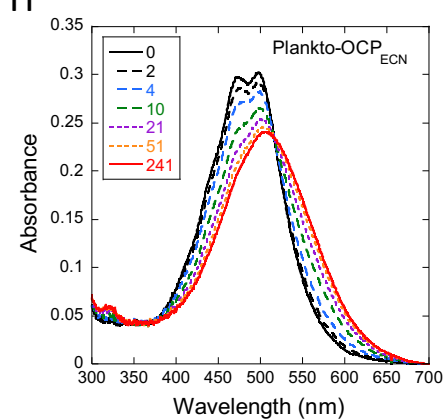

$B$

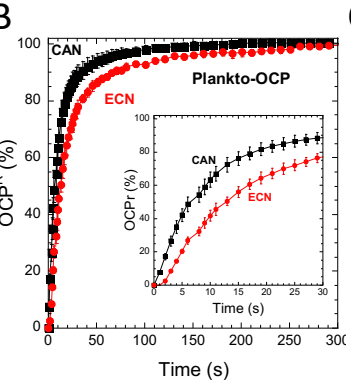

C

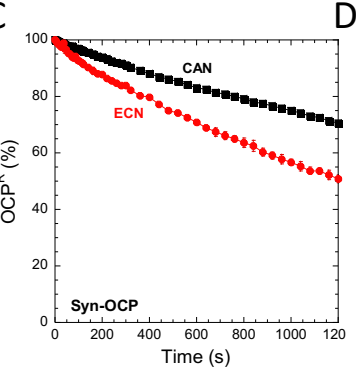

D

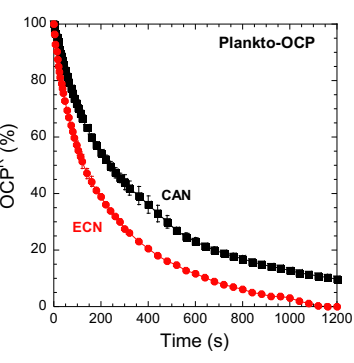

$\mathrm{F}$

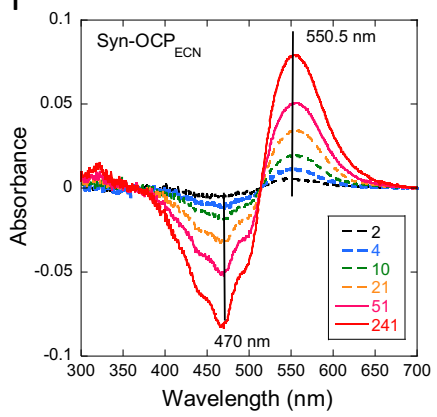

I

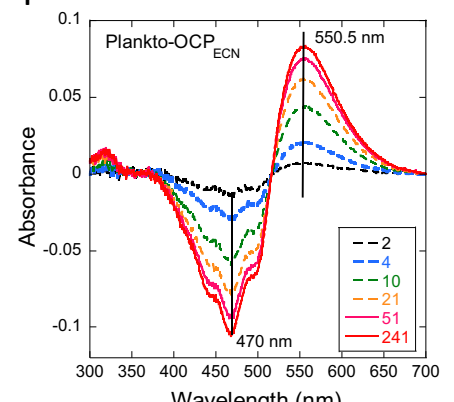

G

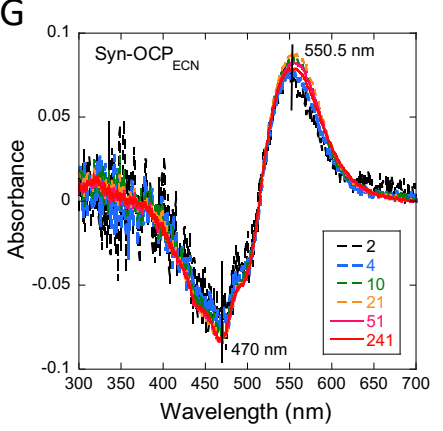

J

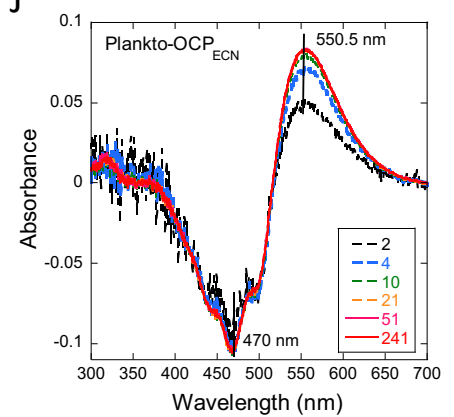

Figure 4: Effect of the nature of the functionalizing ketocarotenoid on photoactivation and recovery of N-terminus His-tagged Synechocystis and Planktothrix OCPs. (A-B) Kinetics of photoactivation of Syn-OCPs (A) and Plankto-OCPs (B) functionalized with ECN (red) or CAN (black). Inset in $A$ and $B$ : First $30 \mathrm{~s}$ of illumination. The OCPs were illuminated with white light $(5000 \mu \mathrm{mol}$ photons $\left.\mathrm{m}^{-2} \cdot \mathrm{s}^{-1}\right)$ at $9.5^{\circ} \mathrm{C}$. (C and $\left.\mathrm{D}\right)$ Thermal recovery of OCPR in darkness of Syn-OCPs (C) and Plankto-OCPs (D) functionalized with ECN (red) or CAN (black). The accumulation of OCPR and its thermal recovery were followed by increase and decrease of absorbance at $550 \mathrm{n}$, respectively. Error bars: standard deviation. Each curve represents the mean of three independent measurements, respectively $(E$ and $H)$ Absorbance spectra of ECN-Syn $(E)$ and ECN-Plankto OCPs $(H)$ at different times of illumination. ( $F$ and $\mathrm{I}$ ) Raw difference absorbance spectra derived from $\mathrm{E}$ and $\mathrm{H}$ respectively. ( $G$ and $J$ ) Difference absorbance spectra derived from $E$ and $H$, respectively, after normalization on the $470 \mathrm{~nm}$ band.

In agreement with previous reports, we found that $0.15 \mathrm{ps}$ after excitation, the $\mathrm{S}_{2}$ excited state has already started to decay. Transient absorption spectra are characterized by a ground state bleaching (GSB) negative band with an extremum at $\approx 500 \mathrm{~nm}$, indicative of $\mathrm{OCP}^{\circ}$ 
depopulation, and by positive absorption (ESA) bands for the $\mathrm{S}_{1}, \mathrm{ICT}$ and $\mathrm{S}^{*}$ states peaking at $\approx 660 \mathrm{~nm}, \approx 750 \mathrm{~nm}$ and $\approx 575 \mathrm{~nm}$ (shoulder), respectively. All excited- and vibrationally-hot ground-states have decayed by the 30 ps time-delay, and only a broad positive band centered at $\approx 560 \mathrm{~nm}$, previously assigned to the photoproduct $\mathrm{P}_{1}$ [19] (Nizinski et al., submitted; doi.org/10.1101/2021.12.26.474187), can be seen at the 95 ps time delay. $\mathrm{P}_{1}$ shows no spectral evolution in our experimental time window, i.e., up to 1 ns time delay.

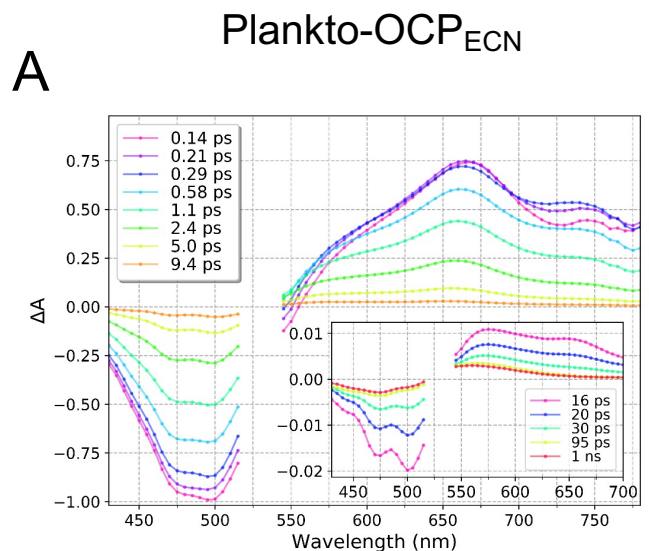

C

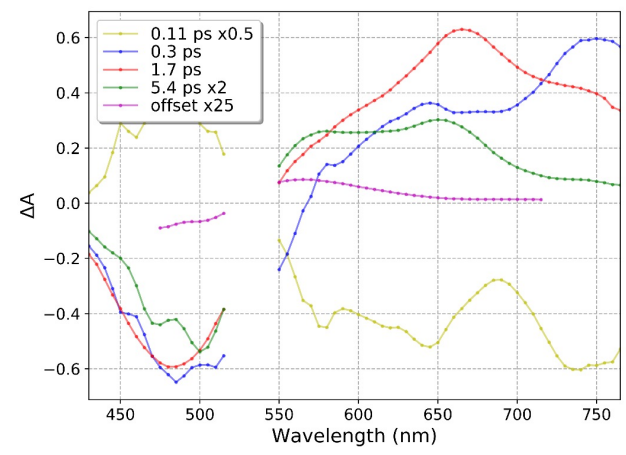

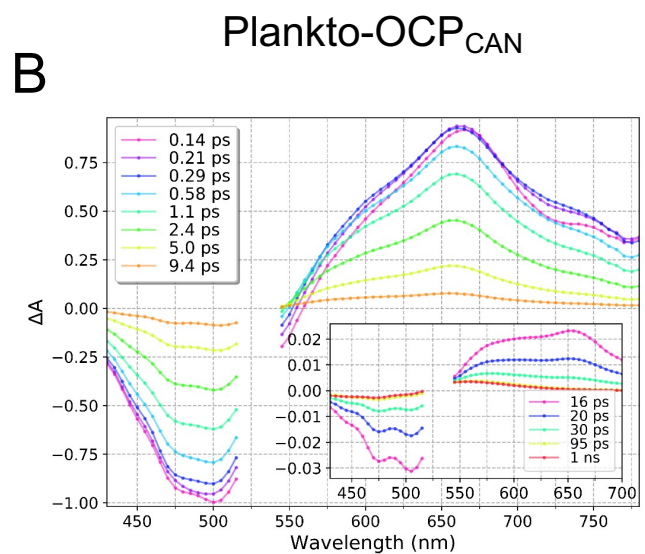

D

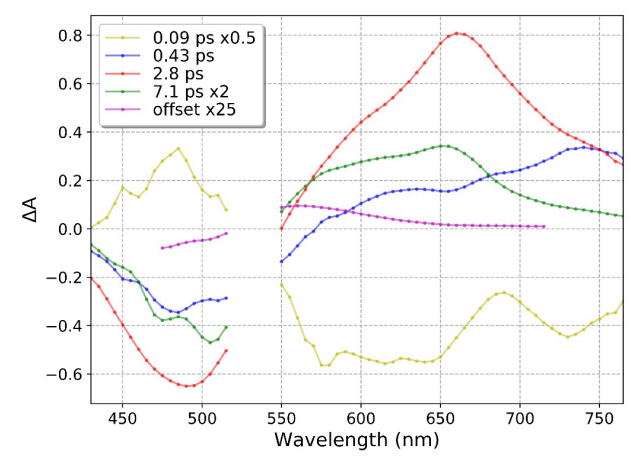

Figure 5: Femtosecond transient absorption data collected on the Ntag-Plankto-OCP. (A, B) Transient absorption spectra measured after excitation at $532 \mathrm{~nm}$ are shown for time delays ranging between 0.14 and $1 \mathrm{~ns}$ for (A) Ntag-Plankto-OCP $\mathrm{ECN}_{\text {and }}$ (B) Ntag-Plankto-OCPCAN. All datasets were normalized to -1 at bleaching minimum, in both spectral and temporal dimensions. (C, D) Decay Associated Spectra (DAS) obtained from the global fit of the transient absorption data spectra shown in (A) and (B), respectively. Data were fitted using four exponential components convolved by a Gaussian pulse (IRF, 110 fs FWHM) and an offset (long-lived photo-product $>10 \mathrm{~ns}$ ).

Four exponential components $\left(S_{2}, I C T, S_{1}\right.$ and $\left.S^{*}\right)$ and one offset $\left(P_{1}\right)$ were accounted for in the global decay analysis, enabling to extract the decay associated spectra (DAS) and lifetimes of the various intermediate states in ECN- and CAN- functionalized Plankto-OCP (Figure 5C and 5D) and Syn-OCP (Supplementary Figure 2C and D). Note that the DAS associated with the shortest time-constants is estimated from a convolution of the $S_{2}$ decay and rise of the excited-state signals, as our resolution is about $110 \mathrm{fs}$. The difference spectrum obtained $\approx 30$ ps post-excitation (offset value in the sum of exponentials) is attributed to the $\mathrm{P}_{1}$ intermediate 
state. Using the GSB kinetics, and assuming that the $S_{1}$, ICT and $S^{*}$ states form from $S_{2}$ and parallelly decay to the ground state, we could further estimate the quantum yields of each, as well as of $\mathrm{P}_{1}$. In agreement with previous reports, the picosecond excited state dynamics and yields are similar for the four tested OCPs, with similar DAS observed for the ICT (blue), $\mathrm{S}_{1}$ (red), $S^{*}$ (green) and $\mathrm{P}_{1}$ (magenta) states, respectively (Figure 5C and 5D). However, a detailed analysis of DAS for CAN and ECN-OCPs (either Plankto or Syn) shows that the value of absorbance amplitude for $\mathrm{S}_{1}$ and ICT above $700 \mathrm{~nm}$ is higher for ECN, assigned to a more pronounced ICT character in the ICT and $S_{1}$ states. The characterized lifetimes are also in agreement with previous reports [59], viz. $\sim 0.15( \pm 0.1), \sim 0.5( \pm 0.1), \sim 2( \pm 0.5)$ and $\sim 7( \pm 2.5)$ ps for the $S_{2}, I C T, S_{1}$ and $S^{*}$ states, respectively (Figure 5 and Table 1 ). Thus, the lifetimes of excited-states do not vary as a function of the species or the functionalizing carotenoid. As expected from the DAS and the literature [40], the formation QY for the ICT state is lower in OCP $P_{\text {CAN }}$. The observed $P_{1}$ yield is yet similar in the four tested OCP $(\approx 0.5 \pm 0.1 \%)$, in agreement with our recent results (Nizinski et al., submitted; doi.org/10.1101/2021.12.26.474187), as well of others [19, 23].

Having excluded the hypothesis that the type of carotenoid or OCP scaffold significantly influences the yield of $\mathrm{P}_{1}$, we shifted our focus to the ns-s dynamics by performing nanosecond transient absorption experiments, whereby a nanosecond laser pulse is used to trigger excitation and the photoactivation outcome is probed in the $50 \mathrm{~ns}-1 \mathrm{~s}$ time window by monitoring changes in the absorbance at $550 \mathrm{~nm}$ (Figure 6). Indeed, on these timescales, the maximum difference-absorbance varies between $563 \mathrm{~nm}$, characteristic of the $\mathrm{P}_{1}$ state, and $550 \mathrm{~nm}$, signing for $O C P^{R}$. Intermediate states were assigned on the basis of earlier reports, with the $P_{1}, P_{2}-P_{2}$, and $P_{3}\left(P_{N}\right) / P_{M} / P_{x}$ states displaying lifetimes of $\sim 50 \mathrm{~ns}, \sim 0.5-10 \mu$ s, and $\sim$ 1-100 ms, respectively. Recall that these states were proposed to be associated with (i) rupture of $\mathrm{H}$-bonds between the carotenoid and the protein scaffold $\left(\mathrm{P}_{1}\right)$; (ii) translocation of the carotenoid from the NTD/CTD interface into the NTD $\left(\mathrm{P}_{2}-\mathrm{P}_{2}, \mathrm{P}_{3}\right)$; and (iii) NTE and CTT detachment $\left(\mathrm{P}_{\mathrm{M}}\right)$ followed by dissociation of the two domains $\left(\mathrm{P}_{\mathrm{x}}\right)$, respectively. $A$ last conformational change thence occurs, yielding from $\mathrm{P}_{\mathrm{X}}$ the metastable $\mathrm{OCP}^{\mathrm{R}}$ (Figure 1). This last step could correspond to the repositioning of the CTT on the CTD-half of the carotenoid tunnel [34]. Note that partial recovery of the $\mathrm{OCP}^{\circ}$ state occurs at all steps (see Figure 1), explaining the decrease in absorbance at $550 \mathrm{~nm}$ over the probed time window. 

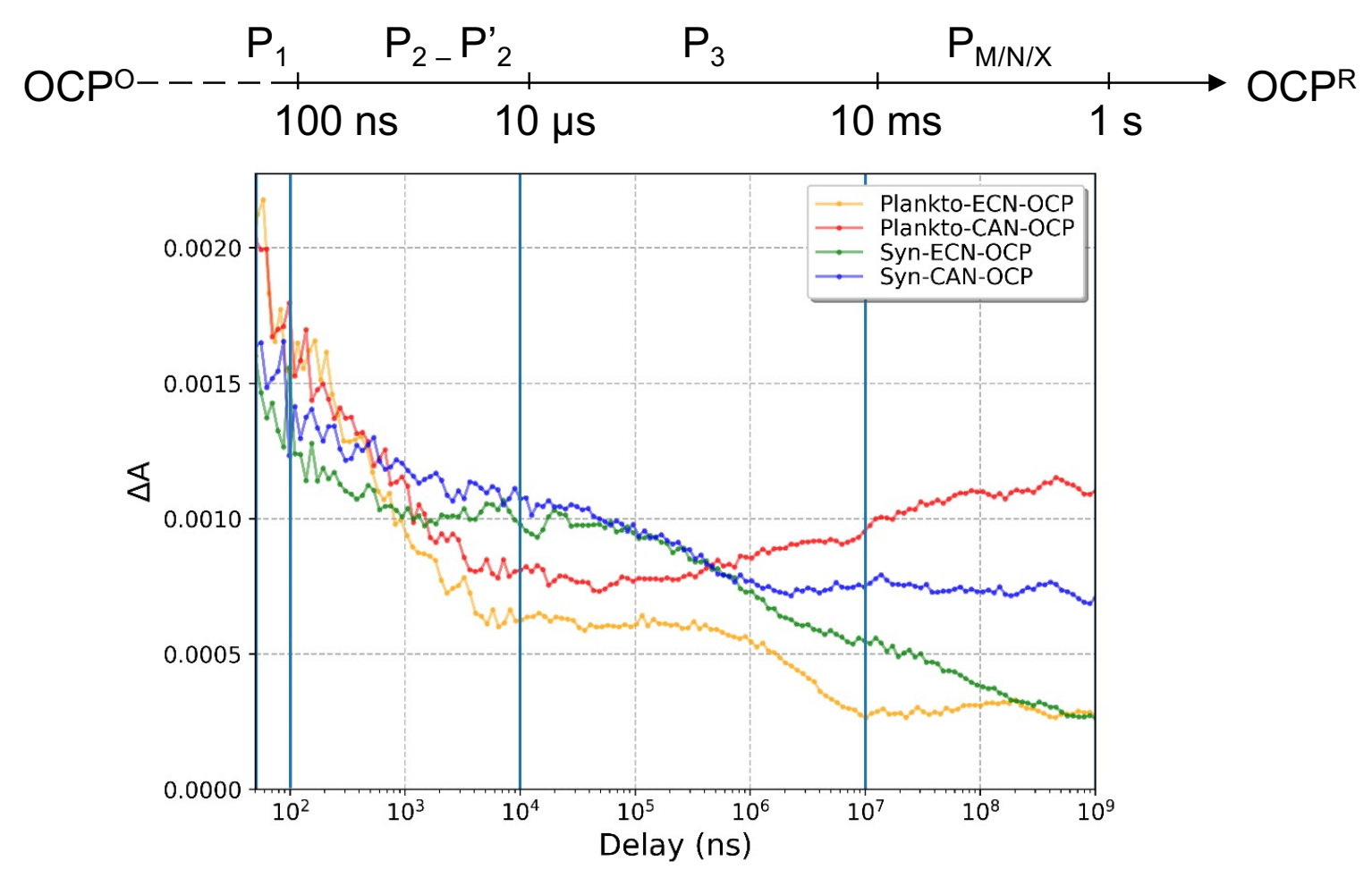

Figure 6: Nanosecond-second dynamics in CAN- and ECN-functionalized Plankto-OCP and Syn-OCP. Time evolution (50 ns to $1 \mathrm{~s}$ ) of the difference absorption signals at $550 \mathrm{~nm}$ recorded on Plankto-OCP ECN $_{\text {(yellow), Plankto-OCP }}$ CAN (red), Syn-OCPECN (green) and Syn-OCPCAN (blue) after excitation by a $532 \mathrm{~nm}$ ns-pulse. The intermediate states proposed $[12,19,23]$ to underlie the observed absorption changes are indicated at the top of the figure. Measurements were carried out at $22^{\circ}$.

Clear differences are seen between the four tested OCPs in these experiments. First, irrespective of the carotenoid, the starting difference absorbance signal (at $50 \mathrm{~ns}$ ) is higher for Plankto-OCP ( 0.002) than for Syn-OCP ( 0.0015), suggesting a higher $\mathrm{P}_{1}$ yield (Figure 6).

This result contradicts the above assumption that the $P_{1}$ yield is the same for all investigated OCPs, but can be rationalized by recalling that (i) in the fs-ns experiments, the GSB band at $490 \mathrm{~nm}$ is used to estimate the $P_{1}$ yield, instead of the characteristic positive absorption band at $563 \mathrm{~nm}$, in the ns-s experiments; and most importantly (ii) a large fraction of $\mathrm{P}_{1}$ reverts to the dark-adapted $\mathrm{OCP}^{\circ}$ state. Thus, the higher $\mathrm{P}_{1}$ yield observed for Plankto-OCP at the start of ns-s transient absorption experiments ( $50 \mathrm{~ns}$ ) could be related to a reduced recovery from $\mathrm{P}_{1}$ of the Plankto-OCPO state. Irrespective of the functionalizing carotenoid, the difference absorptions of Plankto-OCP and Syn-OCP drop by about $60 \%$ and $40 \%$ on the ns - $\mu$ s time scale, respectively. The larger drop in Plankto-OCP signal could underlie sub-optimal translocation of the carotenoid into the Plankto-NTD, compared to the Syn-NTD. On the $\mu \mathrm{s}-\mathrm{S}$ time scale, the most important differences between Plankto-OCP and Syn-OCP are visible in the $\mu \mathrm{s}$ to ms timescale, whereas differences between CAN- and ECN-functionalized OCP concentrate in the ms-s time window. Thus, both carotenoid translocation and NTE/CTT 
detachments seem to be affected by the change in protein scaffold, however, it is domain dissociation that is most affected by a change in the functionalizing carotenoid. This step appears to be faster and more efficient in CAN-functionalized OCP, with a slight increase in $\Delta \mathrm{A}(550 \mathrm{~nm})$ being visible after $1 \mathrm{~ms}$, whereas recovery to the initial state is higher for $\mathrm{ECN}$ functionalized OCP, as evidenced by the decrease of $\triangle \mathrm{A}(550 \mathrm{~nm})$. Of note, this decline is present only in ECN-functionalized OCPs, in accordance with the observation that CANfunctionalized OCP can be photoactivated more efficiently. In the case of Plankto-OCP $\mathrm{CAN}$, we observe faster domain separation and no recovery to $\mathrm{OCP}^{\circ}$ on the $\mu \mathrm{s}-\mathrm{s}$ timescale $(100 \%$ efficiency from $\mathrm{P}_{3}$ to final $O C P^{\mathrm{R}}$ ). The larger difference absorption signals observed for PlanktoOCP (most notably Plankto-OCP $\mathrm{CAN}_{\text {) }}$ on the $10 \mathrm{~ms}-1 \mathrm{~s}$ time window mirror the differences in photoactivation efficiency observed in stationary irradiation experiments.

Multiscale exponential fitting was carried out to extract the lifetimes of the intermediate states (Table 1). The lifetime of the $\mathrm{P}_{1}$ state was found to be almost independent of the OCP variant. For the $\mathrm{P}_{2}-\mathrm{P}_{2}$, states (i.e., on the $50 \mathrm{~ns}$ to $10 \mu \mathrm{s}$ time scale), data were fit by one or two exponentials, depending on the case, yielding lifetimes in the order of 0.5-2 $\mu$ s. Likewise, for the $P_{3} / P_{N} / P_{x}$ states (i.e., on the $0.1-10 \mathrm{~ms}$ time scale), either one or two exponentials were required to fit the data, yielding lifetimes in the order of $0.1-1 \mathrm{~ms}$. Note that these lifetimes are in good agreement with those reported earlier based on experiments carried out on the Ctagged Syn-OCP $\mathrm{ECN}[19,23]$. The experimental setup did not allow to evaluate a lifetime for $O \mathrm{OCP}^{\mathrm{R}}$, but previous reports have pointed to the second timescale [23]. Nevertheless, our data establish that the $O C P^{R}$ yield is higher for CAN-functionalized OCP, and that Plankto-OCP photoactivates more efficiently. Thus, the differences observed in the photoactivation efficiency of CAN- and ECN-functionalized Plankto- and Syn-OCP stem from evolutions observed during the (comparatively-slow) carotenoid translocation, NTE/CTT detachment and domain dissociation steps - but not from changes in the excited-state dynamics of the OCP-embedded carotenoid.

\section{Interaction between OCP and the phycobilisome}

We then inquired the energy-quenching performance of the various ECN- and CANfunctionalized OCPs, focusing on OCP and PBS from Synechocystis and Planktothrix. The PBS from Synechocystis has been well characterized in several laboratories including ours $[42,56]$. However, no information was available regarding the Planktothrix PBS, requiring us to first characterize it biochemically (Supplementary Figure 3). We found that similar to Synechocystis PBS, Planktothrix PBS consists of a core (formed by three cylinders containing four allophycocyanin (APC) trimers) from which radiate six rods constituted of three phycocyanin (PC) hexamers. 
With this characterization in hand, we pursued the investigation of native Syn-OCP and Plankto-OCP energy-quenching activities, focusing first on the effect of the functionalizingcarotenoid (Figure 7) in species-specific OCP/PBS complexes. The decrease of PBS fluorescence was followed using a PAM fluorimeter during incubation of PBS under strong blue-green light and in presence of pre-photoactivated OCPs. Under these conditions, a faster and larger decrease of fluorescence is suggestive of a stronger OCP-PBS interaction. We found that native Syn-OCP $\mathrm{CAN}_{\mathrm{C}}$ and Syn-OCP $\mathrm{ECN}_{\mathrm{EN}}$ induce similar Syn-PBS fluorescence quenching ( $75 \%$ of PBS fluorescence is quenched after $300 \mathrm{~s})$, despite a slightly lower initial

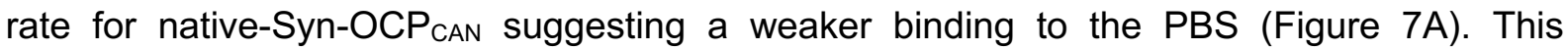
hypothesis was confirmed by the eight times faster PBS fluorescence recovery observed for native-Syn-OCP $\mathrm{PAN}_{\mathrm{C}}$, as compared to native-Syn-OCP $\mathrm{PCN}_{\mathrm{EN}}$ (Figure 7B). The nature of the functionalizing-carotenoid also had an effect on native-Plankto-OCP quenching efficiency and on the Plankto-PBS fluorescence recovery rate. PBS fluorescence quenching was twice more efficient with native-Plankto-OCP $\mathrm{ECN}_{\mathrm{N}}$ than with native-Plankto-OCP $\mathrm{CAN}_{\mathrm{N}}(75$ and $40 \%$ of fluorescence quenching after $300 \mathrm{~s}$ incubation, respectively). In line with the hypothesis that this variance originates from a reduced affinity for the PBS of CAN-functionalized OCPs, fluorescence recovery was nearly three times faster for native-Plankto-OCP $\mathrm{CAN}_{\text {than }}$ nativePlankto-OCP $\mathrm{ECN}_{\text {. }}$

A

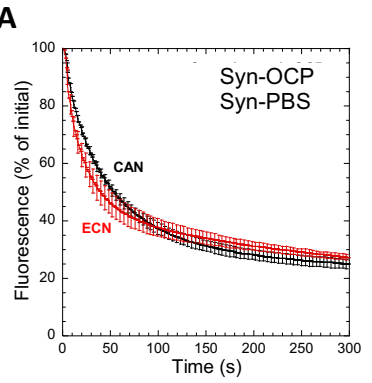

B

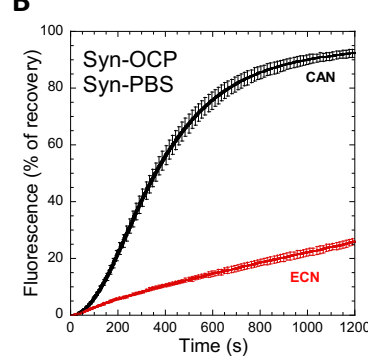

C

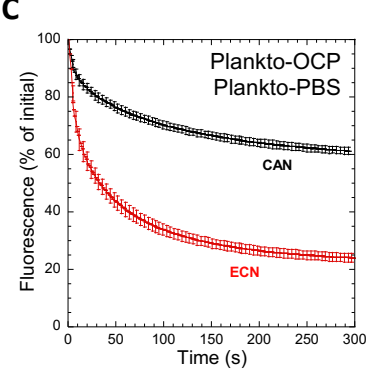

D

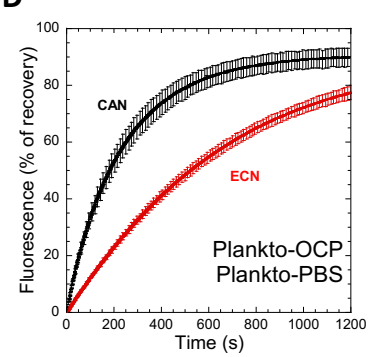

Figure 7: Effect of ketocarotenoid on OCP-PBS interaction. (A, C) Synechocystis (A) and Planktothrix (C) PBS fluorescence quenching induced at $23^{\circ} \mathrm{C}$ in $0.5 \mathrm{M}$ phosphate buffer by CAN (black) and ECN (red) functionalized Synechocystis (A) and Planktothrix (C) native (non-tagged) OCPs. The OCP was pre-photoactivated by illumination with a strong white light $(5000 \mu \mathrm{mol}$ photons $\mathrm{m}^{-2} \cdot \mathrm{s}^{-1}$ ) at $4^{\circ} \mathrm{C}$. The ratio OCP:PBS was 40:1. (B, D) Dark recovery of fluorescence in Synechocystis (B) and Planktothrix (D) phycobilisomes. $100 \%$ of fluorescence in all graphs is the initial fluorescence of phycobilisomes without quenching. Error bars: standard deviation. Each curve represents the mean of three independent measurements.

We also investigated the effect of a His-tag, present either at the $\mathrm{N}$ - or C-terminus, on the species-specific PBS-fluorescence quenching efficiency and recovery rate (Figure 8). We found that irrespective of the species (i.e. for both Syn-OCP + Syn-PBS and Plankto-OCP + Plankto-PBS), His-tagged OCPs are more efficient at inducing PBS-fluorescence quenching, 
with $\mathrm{C}$-tagged OCP further surpassing the $\mathrm{N}$-tagged variants (Figure $8 \mathrm{~A}$ and $8 \mathrm{~B}$ ). This effect is most clear when considering the native, $\mathrm{N}$-tagged and $\mathrm{C}$-tagged Plankto-OCP/PBS complexes. A possible rationalization could come from the higher stabilization of the C-tagged $O C P^{R}$ state. However, this stabilization is not sufficient to explain the observed drastic difference in PBS fluorescence-recovery rates. Indeed, up to $20 \%$ of the initial PBS fluorescence is recovered after 20 min incubation in the dark with the C-tagged Plankto-OCP, which is at variance with the full recoveries observed when native or N-tagged Plankto-OCP and Syn-OCP are used. Thus, the C-terminal His-tag not only stabilizes $\mathrm{OCP}^{\mathrm{R}}$, but also the $O C P^{R}$ - PBS complex.

A

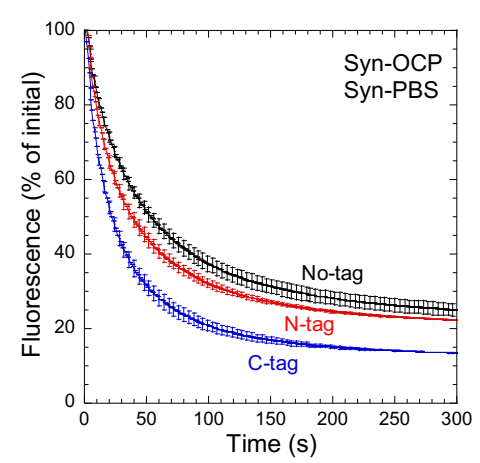

D

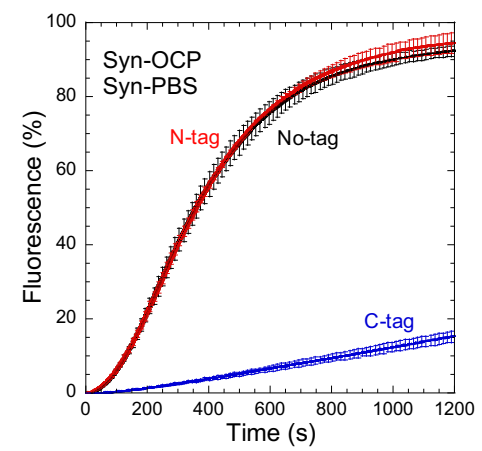

B

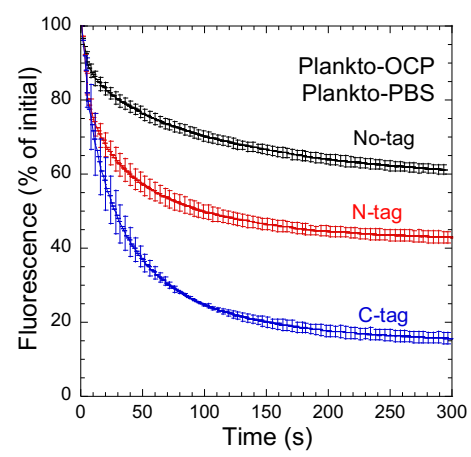

$\mathrm{E}$

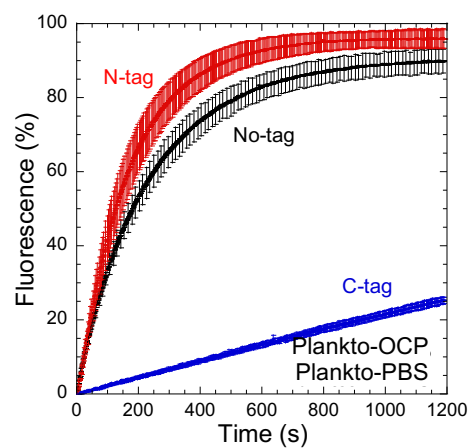

C

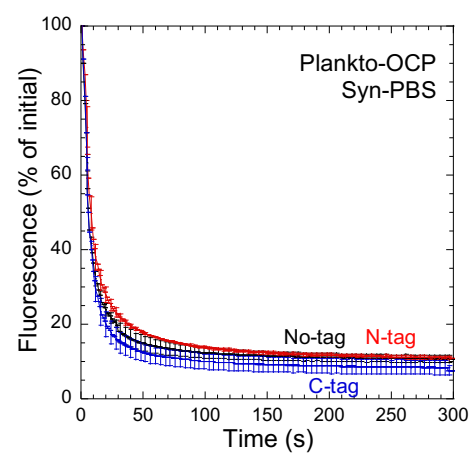

$\mathrm{F}$

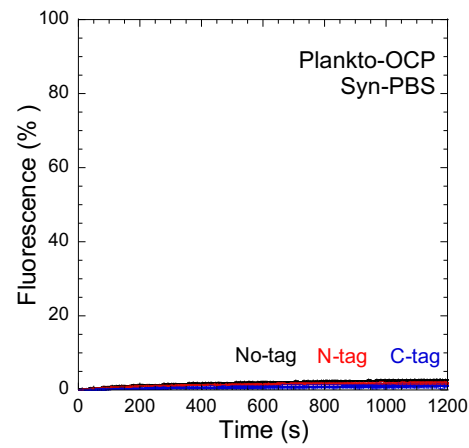

Figure 8: Effect of the presence and position of a His-tag on OCP-PBS interaction. (A-C) Synechocystis (A, C) and Planktothrix (B) PBS fluorescence quenching induced at $23^{\circ} \mathrm{C}$ in $0.5 \mathrm{M}$ phosphate buffer by native (non-tagged; black), N-tagged (red) and C- tagged (blue) CANfunctionalized Synechocystis (A) and Planktothrix (B, C) OCPs. The OCP was pre-photoactivated by illumination of strong white light $\left(5000 \mu \mathrm{mol}\right.$ photons $\left.\mathrm{m}^{-2} . \mathrm{s}^{-1}\right)$ at $4^{\circ} \mathrm{C}$. The ratio OCP:PBS was 40:1. (D-F) Dark recovery of fluorescence in Synechocystis (D, F) and Planktothrix (E) PBS. $100 \%$ of fluorescence in all graphs is the initial fluorescence of phycobilisomes without quenching. Error bars: standard deviation. Each curve represents the mean of three independent measurements.

In the past, we demonstrated that Synechocystis, Arthrospira and Anabaena OCPs bind more strongly to the Synechocystis PBS than to the Anabaena or Arthrospira PBS [42], with Synechocystis OCP binding comparatively weakly to the PBS from other species [42]. Hence, we asked whether or not these conclusions hold as well for Planktothrix OCP and PBS. We 
found that irrespective of the presence and position of the His-tag, Planktothrix OCP binds more strongly to Synechocystis PBS than to Planktothrix PBS, with all Plankto-OCPs inducing a faster and more efficient fluorescence quenching of Syn-PBS (Figure 8B) compared to Plankto-PBS (Figure 8C). Furthermore, the Synechocystis PBS fluorescence recovery was virtually null, indicating that the complex formed by Plankto-OCP and Synechocystis-PBS is highly-stable at $0.5 \mathrm{M}$ phosphate (Figure $8 \mathrm{~F}$ ). Contrastingly, Syn-OCP was unable to induce Planktothrix PBS fluorescence even at higher phosphate concentrations (till 1.4 M phosphate), suggesting that it is able to interact only with Synechocystis PBS.

\section{Structures of CAN- and ECN-functionalized Planktothrix OCP reveal new features.}

The crystal structures of Synechocystis PCC 6803 (PDB id: 4xb5), Anabaena PCC 7120 (PDB id: 5hgr; also referred to as Nostoc), Tolypothrix PCC 7601 (PDB id: 6pq1; also referred to as Fremyella diplosiphon) OCP have been solved in the CAN-functionalized states, while those of Synechocystis PCC 6803 (PDB id: 3mg1) and Arthrospira maxima (PDB id: 5ui2; also referred to as Limnospira) were determined in the ECN and hECN functionalized states, respectively. Structural comparison of these could allow to rationalize the faster and more efficient photoactivation observed for CAN-functionalized OCP in transient absorption (Figure 6 ) and photostationary experiments (Figure 4). Structural information on Planktothrix OCP is yet absent, preventing identification of the structural features that could underlie its functional characteristics - notably, the thwarted translocation of carotenoids into the NTD, revealed by transient absorption spectroscopy, and the increased photoactivation rate and lower stability of its $O C P^{R}$ state, revealed by photo-stationary experiments (Figures 4 and 6).

Hence, we set to characterize the structure of Ntag-Plankto-OCP in the CAN- and ECNfunctionalized states. The structure was solved, for each of these, in two space groups, viz. $C 2$ and $P 2_{1}$, revealing a remarkable conservation of the secondary structure (Figure 9A). The C2 structures were solved at slightly higher resolution (1.4 and $1.7 \AA$ resolution for NtagPlankto-OCP $\mathrm{CAN}_{\mathrm{C}}$ and Ntag-Plankto-OCP $\mathrm{PCN}_{\mathrm{EN}}$, respectively) than the $P 2_{1}$ structures $(1.8 \AA$ resolution for both OCPs) (Table 2). In the $P 2{ }_{1}$ space group, the asymmetric unit features the dimer that has been observed in previous structures [8, 17, 21, 30, 57-59] and was identified as naturally-occurring in vitro [60] with a dissociation constant in the order of $17 \mu \mathrm{M}$ depending on reports [16]. The large dimerization interface (1046.7 and $1084.7 \AA^{2}$ of buried surface area (BSA) in the $P 2{ }_{1}$ structures of Plankto-OCP $P_{C A N}$ and Plankto-OCP $\mathrm{ECN}_{\text {) }}$ is mainly supported by $\mathrm{N}$-terminal helices $\alpha \mathrm{A}$ (NTE) and $\alpha \mathrm{B}$, which contribute $\sim 70 \%$ of the $\mathrm{BSA}$, with minor contributions from helix $\alpha \mathrm{H}(\sim 20 \%$ of the BSA) and the $\alpha \mathrm{E}-\alpha \mathrm{F}$ and $\beta 2-\beta 3$ loops $(\sim 10 \%$ of the BSA) (Figure 9B, C and Supplementary Table S1). Interestingly, the relative contributions of $\alpha \mathrm{A}$ and $\alpha \mathrm{B}$ to the $\mathrm{BSA}$ at the dimerization interface vary depending on the functionalizing 


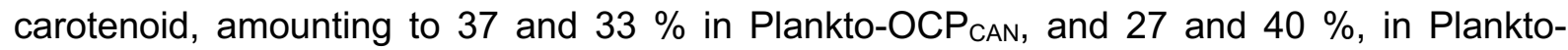
$O C P_{E C N}$, respectively. Additionally, two $\mathrm{H}$-bonds fixing $\alpha \mathrm{A}$ and $\alpha \mathrm{B}$ from facing monomers in the Plankto-OCP $P_{C A N}$ dimer (viz. R9(NH2)-Q30(O) and R9(NH1)-L31(O)) are suppressed in the Plankto-OCP $\mathrm{ECN}_{\mathrm{EN}}$ dimer (Figure 9D and Supplementary Table S1). Thus, the NTE $(\alpha A)$ is less constrained (BSA decreases by $25 \%$ ) at the dimerization interface in the $P 2{ }_{1}$ Plankto-OCP $P_{E C N}$ structure than in the $P 2_{1}$ Plankto-OCP $P_{\text {CAN }}$ structure, but the dimer is more tightly packed, with 25,41 and $52 \%$ increase in the BSA contributed by $\alpha B$ (largest contributor to the dimerization interface in all structures), and the $\alpha \mathrm{E}-\alpha \mathrm{F}$ and $\beta 2-\beta 3$ loops, respectively (Supplementary Table $\mathrm{S} 1)$. The rest of the $\mathrm{H}$-bonding network at the dimerization interface is overall conserved in the $P 2{ }_{1}$ structures, with $3 \mathrm{H}$-bonds between $\alpha B$ and the facing $\alpha \mathrm{H}(\mathrm{N} 14(\mathrm{OD} 1)-\mathrm{A} 133(\mathrm{~N}), \mathrm{T} 15(\mathrm{O})-$ N134(ND2), T17(OG1)-N134(ND2)), one H-bond between $\alpha A$ and the facing $\alpha E F$ loop (either D6(OD2)-T90(OG1) or D6(OD2)-N88(ND2) in the Plankto-OCP $P_{C A N}$ and Plankto-OCP $P_{E C N}$ structures, respectively) and a salt-bridge between facing aB residues (D19(OD2)-R27(NE)) (Supplementary Figures 4 and 5).

In the $C 2$ structures, the dimerization interface is crystallographic $(-\mathrm{x}, \mathrm{y},-\mathrm{z})$ and at the origin of the two-fold symmetry of the crystals. The BSA at the dimerization interface amounts to 1061 and $1023 \AA^{2}$ in the Plankto-OCP ${ }_{C A N}$ and Plankto-OCP $P_{E C N}$ structures, respectively (Supplementary Table S1). We note that in the C2 structures, the relative contributions of $\alpha A$ and $\alpha B$ to the $B S A$ at the dimerization interface hardly vary depending on the functionalizing

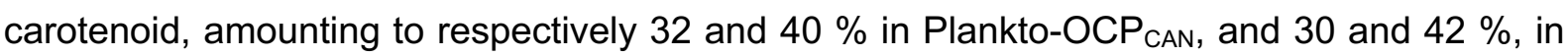
Plankto-OCP $\mathrm{ECN}_{\mathrm{E}}$ (Supplementary Table S1). Nonetheless, alike in the $P 2_{1}$ structures, the two $\mathrm{H}$-bonds affixing $\alpha \mathrm{A}$ and $\alpha \mathrm{B}$ from facing monomers in the C2 Plankto-OCP $\mathrm{CAN}_{\mathrm{N}}$ dimer, i.e., $\mathrm{R} 9(\mathrm{NH} 2)-\mathrm{Q} 30(\mathrm{O})$ and $\mathrm{R} 9(\mathrm{NH} 1)-\mathrm{L} 31(\mathrm{O})$, are absent in the C2 Plankto-OCP $\mathrm{ECN}_{\text {dimer. Thus, }}$ these $\mathrm{H}$-bonds are present in both the $P 2_{1}$ and $C 2$ Plankto-OCP ${ }_{\text {CAN }}$ structures, but in neither of the Plankto-OCP $\mathrm{ECN}_{\mathrm{N}}$ structures. The rest of the $\mathrm{H}$-bonding network at the dimerization interface is again overall preserved in the two $\mathrm{C} 2$ structures, with two to three $\mathrm{H}$-bonds between $\alpha A$ and the facing $\alpha H$ (T15(O)-N134(ND2) and T17(OG1)-N134(ND2) in the two structures, and $\mathrm{N} 14(\mathrm{OD} 1)$ to $\mathrm{A} 133(\mathrm{~N})$ in the Plankto-OCP $\mathrm{PAN}_{\mathrm{C}}$ structure only), one $\mathrm{H}$-bond between $\alpha \mathrm{A}$ and the facing $\alpha E-\alpha F$ loop (D6(OD2)-T90(OG1) in the two structures and a salt-bridge between facing $\alpha B$ residues (D19(OD2)-R27(NE)) (Figure 9D). The latter salt-bridge is preserved among all OCP structures reported thus far, suggesting that it is a defining interaction in the naturally-occurring OCP dimer. Indeed, it was shown that mutation into a leucine of R27 yields a constitutively monomeric OCP [16]. The extent of the biological dimerization interface is also overall preserved among all available OCP structures including ours, with a mean BSA of 1051 $\pm 90 \AA^{2}$ (viz. 6pq1, 5ui2, 5hgr, 3mg1, 4xb5, and 7qd0, 7qcZ, 7qd1 and 7qd2). In this context, 
two structures stand out, viz. the Tolypothrix $\mathrm{OCP}_{\mathrm{CAN}}$ and Arthrospira $\mathrm{OCP}_{\mathrm{hECN}}$ structures, with BSA of 857.9 and $1186.1 \AA^{2}$, respectively (Supplementary Tables S1 and S2).

A

C2, CAN, chain A

$\mathrm{P} 2$, CAN, chain $\mathrm{A}$

$\mathrm{P} 2{ }_{1}, \mathrm{CAN}$, chain $\mathrm{C}$

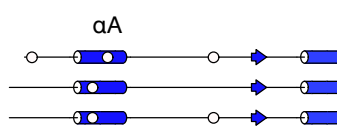

$\mathrm{aB}$

$\mathrm{aC}$

$\mathrm{aD}$

C2, ECN, chain A

$\mathrm{P} 2$, $\mathrm{CAN}$, chain $\mathrm{A}$

$P 2{ }_{1}, C A N$, chain $C$

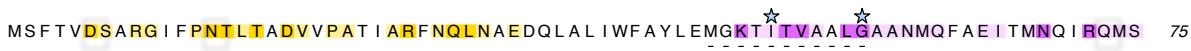
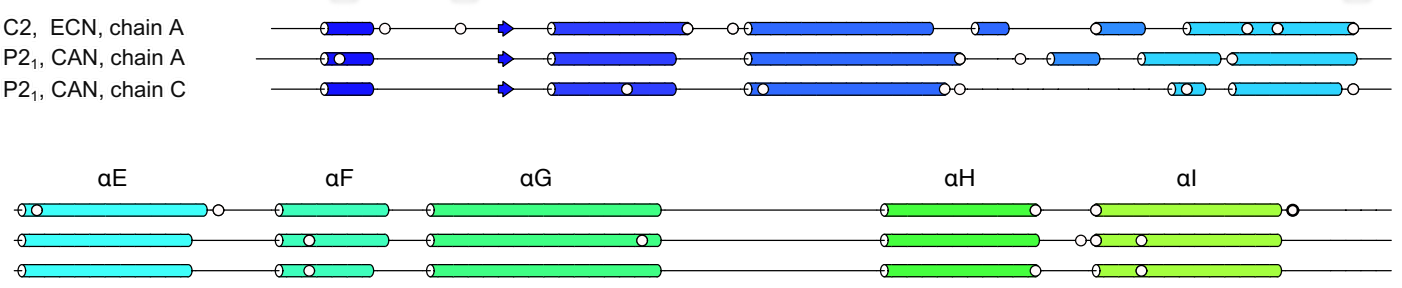

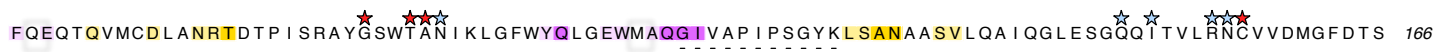

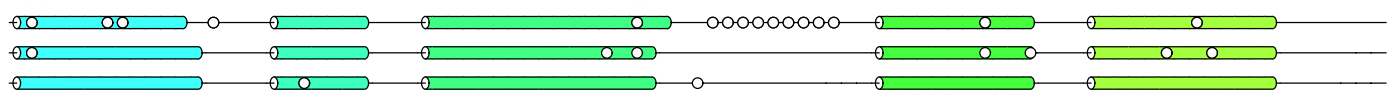

$\longrightarrow$

KLDSSQRVSEPVVVPRDMAQRTQVT I EGI DNPTVLNYMNNMNANDFEVLIELFTPDGALQPPFQKP IVGKDAVLRFFREECQNLKL I PERG 257

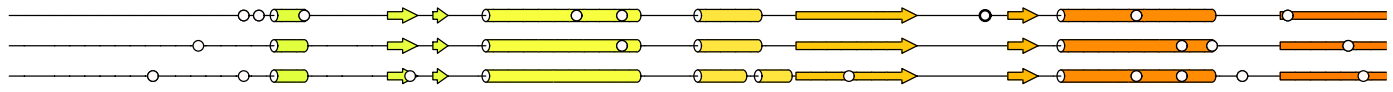

$\underset{a \rightarrow 0+\infty}{\Rightarrow}$

VTEPADGGYTQI KVTGKVQTPWFGAGVGMNMAWRFLLSPENK I FFVA I DLLASPKELLNLVR 319

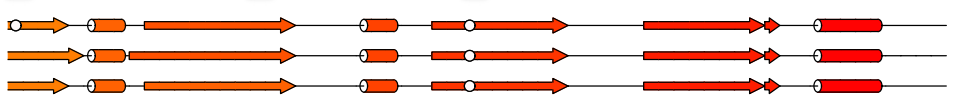

B

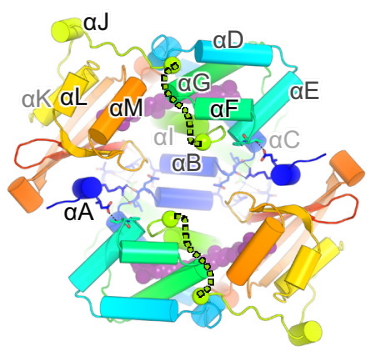

E

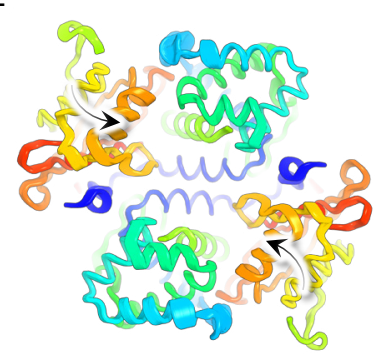

C

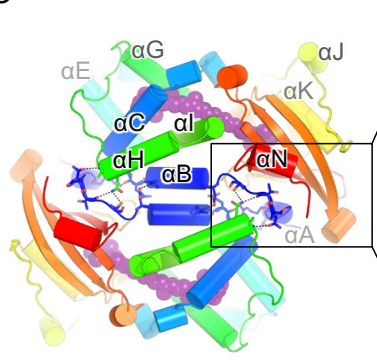

F

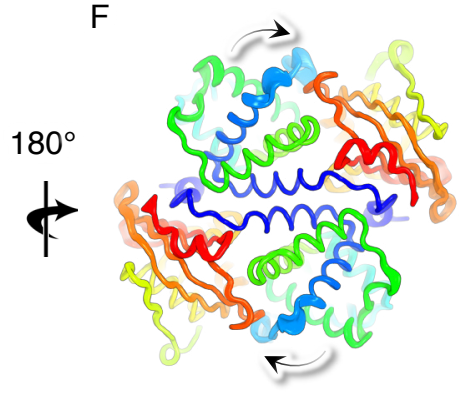

D

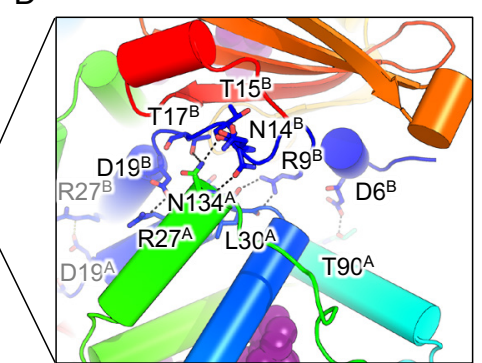

G

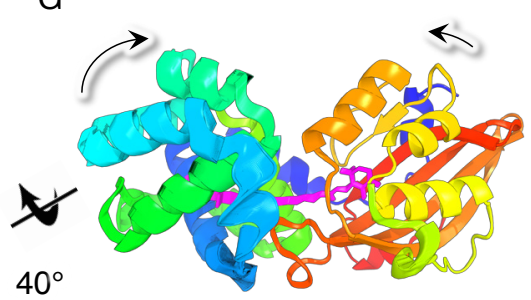


Figure 9. The naturally occurring OCP dimer is found in all OCP crystals, including those of Plankto-OCP. Residues involved in the dimerization are highlighted in yellow, whereas residues involved in the formation of interface $X$ are highlighted in purple. Dark and light colouring indicate residues involved in polar and van der Walls interactions, respectively. Squares highlight residues present in alternate conformations in at least $50 \%$ of the analyzed Plankto-OCP chains. (A) The secondary structure of OCP is overall well conserved amongst Plankto-OCP structures obtained in different space groups and with different pigments. White dots highlight residues which are observed in alternate conformations. Blue stars indicate residues which have been shown to play a role in the OCP-PBS interaction, and red stars point to residues that could be at the origin of the stronger attachment of Plankto-OCP to the Syn-PBS. (B-C). The asymmetric unit of the $P 2{ }_{1}$ crystals features a dimer (here shown as a ribbon with the two facing monomers colored sequence-wise, from cold $(\mathrm{N}$ ter) to hot (C-ter) colors), whereas in $\mathrm{C} 2$ crystals, the dimer is crystallographic, hence only a single monomer is found in the asymmetric unit. (D) Polar contacts at the dimerization interface involve a conserved salt-bridge between D19 and R27, as well as conserved $\mathrm{H}$-bonds between facing D6 and T90, and between facing N134 and N14, T15, and T17. (E, F, G) The C2 structures display a more compact conformation than the $P 2{ }_{1}$ structures, at both the dimer $(\mathrm{E}, \mathrm{F})$ and at the monomer levels $(G)$. The figure illustrates the trajectory followed by Plankto-OCP as we interpolate from the C2-CAN to the $P 2{ }_{1}$-CAN structure, highlighting the secondary structure elements which diverge most upon packing in the two crystal types. Arrows show the overall direction travelled by domains as we interpolate between the C2-Can and $P 2{ }_{1}$-CAN Plankto-OCP.

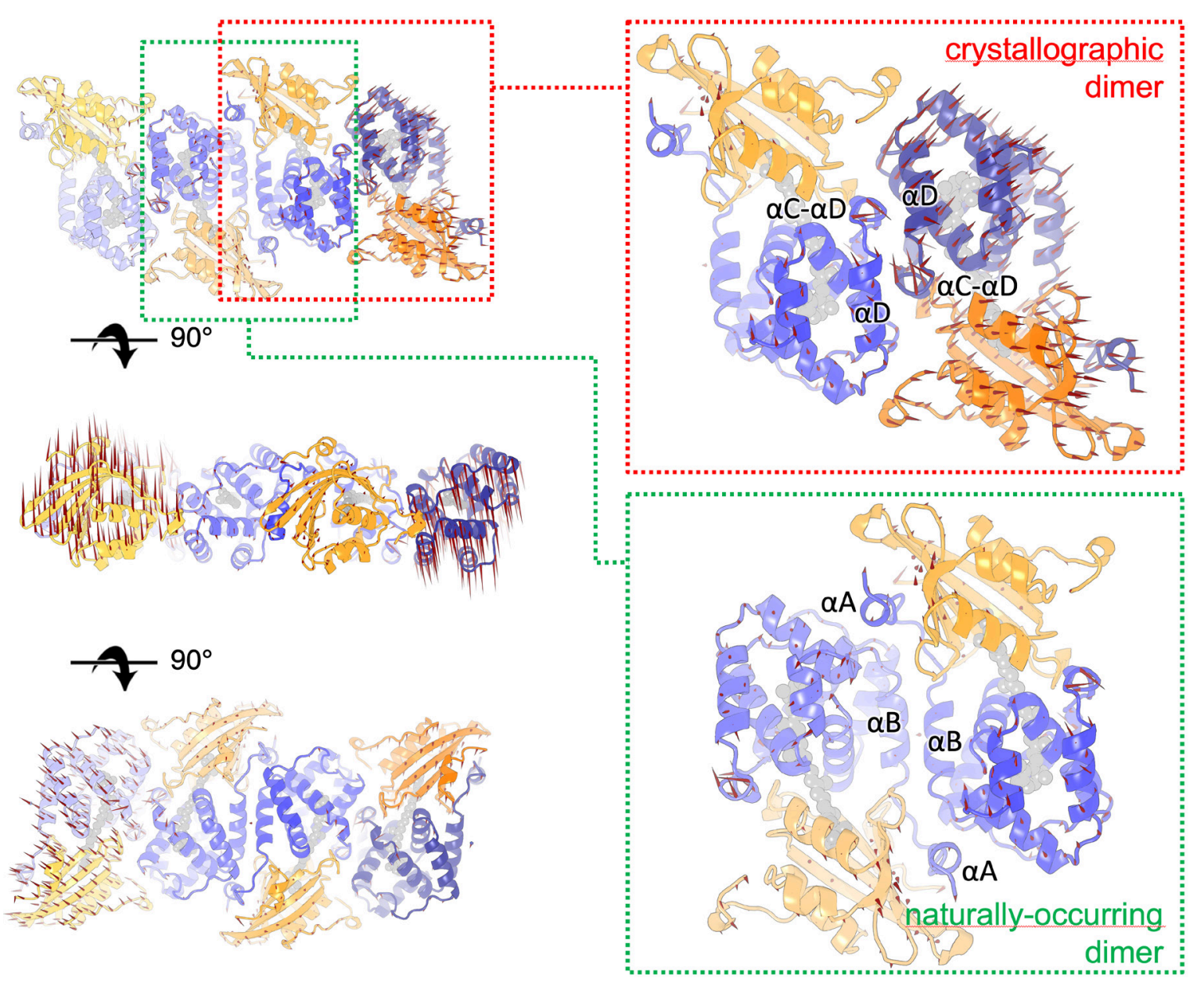

Figure 10: The Planktothrix OCP features two similarly large packing interfaces in crystals. In Plankto-OCP crystals, a large interface, additional to the dimerization interface (Table S1), is found which we coined interface $X$ (Table S3). This interface, mainly contributed by by helices $\alpha D, \alpha G$ and 
$\alpha \mathrm{J}$ and by the $\alpha \mathrm{C}-\beta 4$ and $\beta 5-\beta 6$ loops, matches the dimerization interface, in $P 2{ }_{1}$ crystals (BSA of $\sim 1050 \AA^{2}$ ), but largely exceeds it, in the $C 2$ crystals (BSA of $\sim 1600 \AA^{2}$ ). Changes in the extent of interface $X$ result in a reorientation of domains in each monomer forming the naturally-occurring dimer, and in an increase in the opening angle between monomers, in the dimer. Arrows indicate the direction and distance along which $C \alpha$ atoms travel as we interpolate from the $P 2{ }_{1}$ to the $C 2$ crystals.

Further analysis of the crystalline interfaces reveals that differences between the $P 2_{1}$ and $C 2$ crystals originate at a second large interface, absent in previously characterized OCP structures (Figure 10). This additional interface, coined interface $X$, corresponds to the dimerization interface in the $P 2_{1}$ crystals (BSA of 1001.6 and $1054.6 \AA^{2}$ in the Ntag-Plankto$\mathrm{OCP}_{\mathrm{CAN}}$ and Ntag-Plankto-OCP $\mathrm{ECN}_{\mathrm{E}}$ structures, respectively) but largely exceeds it in the $\mathrm{C2}$ crystals (BSA of 1650.3 and $1548.7 \AA^{2}$ in the Plankto-OCP $\mathrm{CAN}_{\mathrm{C}}$ and Plankto-OCP $\mathrm{ECN}_{\mathrm{N}}$ structures) (Supplementary Table S3). Interface $X$ involves multiple secondary structure elements, including the $\alpha \mathrm{C}-\mathrm{aD}$ loop $\left(\sim 20\right.$ and $36 \%$ of the overall $\mathrm{BSA}$ in $C 2$ and $P 2{ }_{1}$ crystals, respectively), $\alpha \mathrm{D}$ ( 20 and $10 \%$, respectively), $\alpha \mathrm{E}$ ( 10 and $5 \%$, respectively), $\alpha \mathrm{G}$ and the $\alpha G-\alpha H$ loop (20 and $30 \%$, respectively), the linker (20 and $10 \%$ ), and the $\alpha \mathrm{M}-\beta 4$ ( 5 and 4 $\%$, respectively) and $\beta 5-\beta 6$ ( 2 and $5 \%$, respectively) loops (Supplementary Table S3). Only the first two structural elements contribute $\mathrm{H}$-bonds at interface $\mathrm{X}$ in the $P 2_{1}$ crystals, whereas all of them do in the $C 2$ crystals (Supplementary Table S3). Conformational changes in aG and in the $\alpha \mathrm{C}-\alpha \mathrm{D}$ and $\alpha \mathrm{G}-\alpha \mathrm{H}$ loops, and resultantly changes in the BSA contributed by the $\alpha \mathrm{C}$ $\alpha \mathrm{D}$ loop (+8 and $+24 \%$ in the Ntag-Plankto-OCP $\mathrm{CAN}_{\text {and Ntag-Plankto-OCP }}$ aCN structures, respectively), $\alpha \mathrm{D}$ (-64 and $-68 \%$, respectively), $\alpha \mathrm{E}$ (-70 and $-66 \%$, respectively) and the linker (-74 and $-68 \%$, respectively), explain the shrunken interface $\mathrm{X}$ in the $P 2{ }_{1}$ crystals, compared to the $C 2$ crystals (Supplementary Table S3). These changes in packing translate to changes in the opening-angles at the dimerization (Figure 9E, F, G) and NTD/CTD interfaces (Figure 11), offering a glimpse into the molecular breathing motions that animate $\mathrm{OCP}$, at both the monomer and the dimer levels (Figure 10). Briefly, monomers come closer to one another in the $P 2_{1}$ (asymmetric unit) dimer than in the $C 2$ (crystallographic) dimer (change in openingangle and distance between chains: $-5.6^{\circ}$ and $+1.1 \AA$ for Ntag-Plankto-OCP CAN $;-6.8^{\circ}$ and + $1.2 \AA$ for Ntag-Plankto-OCP $\mathrm{ECN}_{\mathrm{N}}$.

The $P 21$ monomers are nonetheless characterized by an increased opening angle between the NTD and the CTD (Figures 9G, 10 and 11). Chain A shows a larger deviation with respect to the unique chain in C2 crystals (change in opening-angle and distance between the NTD and CTD: $+3.1^{\circ}$ and $+1.0 \AA$ for Ntag-Plankto-OCP $\mathrm{CAN} ;+4.6^{\circ}$ and $+1.0 \AA$ for Ntag-Plankto$\mathrm{OCP}_{\mathrm{ECN}}$ ) than chain $\mathrm{B}$ (change in opening-angle and distance between the NTD and CTD: $+1.3^{\circ}$ and $+0.4 \AA$ for Ntag-Plankto-OCP $\mathrm{CAN} ;+1.4^{\circ}$ and $+0.3 \AA$ for Ntag-Plankto-OCP $\mathrm{ECN}_{\text {) }}$ (Figure 9). As a result, the predicted radii of gyration $(\mathrm{Rg})$ for the $P 2_{1}$ asymmetric unit dimers $(26.16$ 
and 26.12 $\AA$ for Ntag-Plankto-OCP $\mathrm{PAN}_{\mathrm{CN}}$ and Ntag-Plankto-OCP $\mathrm{ECN}_{\mathrm{N}}$, respectively) are larger than those predicted for the $C 2$ crystallographic dimers (26.01 and $25.90 \AA$ for Ntag-Plankto-

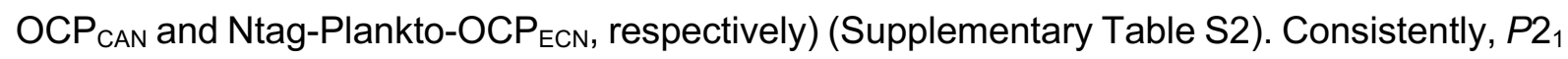
chain $B$ features a structure that is intermediate between $P 2{ }_{1}$ chain $A$ and the unique $C 2$ chain (change in opening-angle and distance between the NTD and CTD in $P 2{ }_{1}$ chain A with respect to $P 2{ }_{1}$ chain $\mathrm{B}:+3.3^{\circ}$ and $+0.9 \AA$ for Ntag-Plankto-OCP $\mathrm{CAN} ; 4.0^{\circ}$ and $+0.7 \AA$ for Ntag-Plankto$\mathrm{OCP}_{\mathrm{ECN}}$ ) (Figure 11A). This is visible also in the predicted $\mathrm{Rg}$ for the various chains $(20.81$, 20.67 and $20.52 \AA$ for Ntag- $P 2_{1}$ chain $A, P 2_{1}$ chain $B$ and $C 2$ Plankto-OCP ${ }_{C A N}$, respectively; and $20.73,20.50$ and $20.48 \AA$ for the Ntag-Plankto-OCP $\mathrm{ECN}_{\mathrm{EN}}$ counterparts, respectively) (Supplementary Table S2). Altogether, these changes affect the positioning of the carotenoid which, despite preservation of $\mathrm{H}$-bonds from its carbonyl oxygen to $\mathrm{Y} 203(\mathrm{OH})(2.5-2.8 \AA$ distance between non-hydrogen atoms) and W290(NH1) (2.8-3.0 $\AA$ distance between nonhydrogen atoms) and a quasi-perfect alignment of its $\beta 1$-ring in the CTD, tilts towards Y44 in the $P 2{ }_{1}$ structures (Figure 11B). With respect to the $C 2$ Ntag-Plankto-OCP $P_{C A N}$ structure, the tilt angles of the carotenoid are 3.8 and $1.8^{\circ}$ in chains $A$ and $B$ of the $P 2{ }_{1}$ Ntag-Plankto-OCP $P_{E C N}$ structure, 3.2 and $2.9^{\circ}$ in chains $A$ and $B$ of the $P 2{ }_{1}$ Ntag-Plankto-OCP ${ }_{\text {CAN }}$ structure, and $0.8^{\circ}$ in the $\mathrm{C} 2 \mathrm{Ntag}-\mathrm{Plankto}-\mathrm{OCP}_{\mathrm{ECN}}$ structure. Thus, crystal packing traps different conformations of the Ntag-Plankto-OCP monomers, which differ (i) in the conformation displayed, at interface $X$, by $\alpha G$ and the $\alpha C-\alpha D$ and $\alpha G-\alpha H$ loops, (ii) in the opening angle between domains, at the NTD/CTD interface, (iii) in the opening angle between monomers, in the biological dimer; and (iv) in the positioning of the carotenoid (Figures 9-12). It is of important note that despite these changes, and the presence of two alternate side-chain conformations for R155 in the NtagPlankto-OCP $\mathrm{ECN}_{\mathrm{N}}$ structures, the R155-E246 salt-bridge and N104-W279 H-bond, which support the NTD / CTD interface, are preserved in all structures (distance between nonhydrogen atoms 2.8-3.2 $\AA$ ) (Supplementary Figures 4 and 5). We also note that previously determined structures of Synechocystis, Anabaena, Tolypothrix and Arthrospira OCP align best with the $C 2$ Ntag-Plankto-OCP $\mathrm{PAN}_{\mathrm{C}}$ and Ntag-Plankto-OCP $\mathrm{ECN}_{\mathrm{E}}$, suggesting that these should be used for comparisons, rather than the $P 2_{1}$ structures (Supplementary Table S2). In this context, it must be acknowledged that differences in the opening angle between the NTD and the CTD can also be seen from the comparison of the two chains constituting the asymmetric unit dimer in the Anabaena OCP structure (PDB id: 5hgr), with chain A aligning best with the $C 2$ Ntag-Plankto-OCP conformers. We last note that ECN-functionalized OCP monomers and dimers are more compact than the CAN-functionalized counterparts (Figure 11 and Supplementary Table S2), and that a similar trend is visible in the comparison of CANfunctionalized (PDB id: 4xb5; one chain with a predicted radius of gyration of $20.49 \AA$ ) and ECN-functionalized (PDB id: 3mg1; two chains with predicted radii of gyration of 20.34 and 
$20.33 \AA$ ) NtagSyn-OCP structures. In the case of the Plankto-OCP structure, conformational changes again concentrate in the NTD (Figure 12).
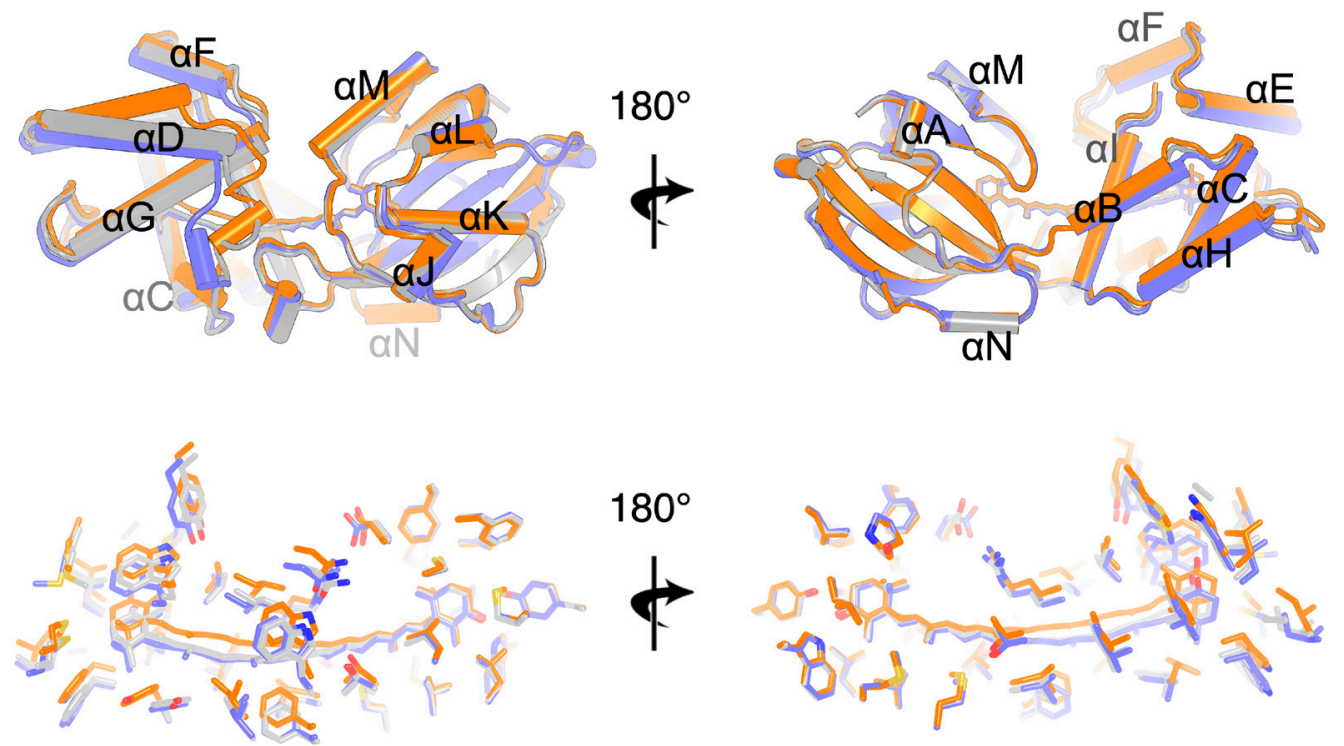

Figure 11: Plankto-OCP structures differ in the compaction of the monomer as well as in the internal structure of the NTD. (A) The $C 2$ (orange), and $P 2{ }_{1}$ chain A (slate) and chain B (grey) are overlaid as ribbons. Structural alignment, performed using the CTD atoms, highlight the change in opening angle between the CTD and the NTD. Indeed, the CTD structure is highly conserved with hardly no conformational changes observed amongst structures. Large differences are yet seen in the NTD, notably in the $\alpha C-\alpha D$ and $\alpha G-\alpha H$ loops, but as well in the relative positioning of $\alpha C$ and $\alpha D$ helices. Here, the Plankto-OCP-CAN structures are shown, but the same observations were made when comparing the Plankto-OCP-ECN structures. (B) Close up view on CAN and on the residues lining the carotenoid tunnel. A change in the orientation of the carotenoid is seen upon compaction of the OCP structure due to change in space group.
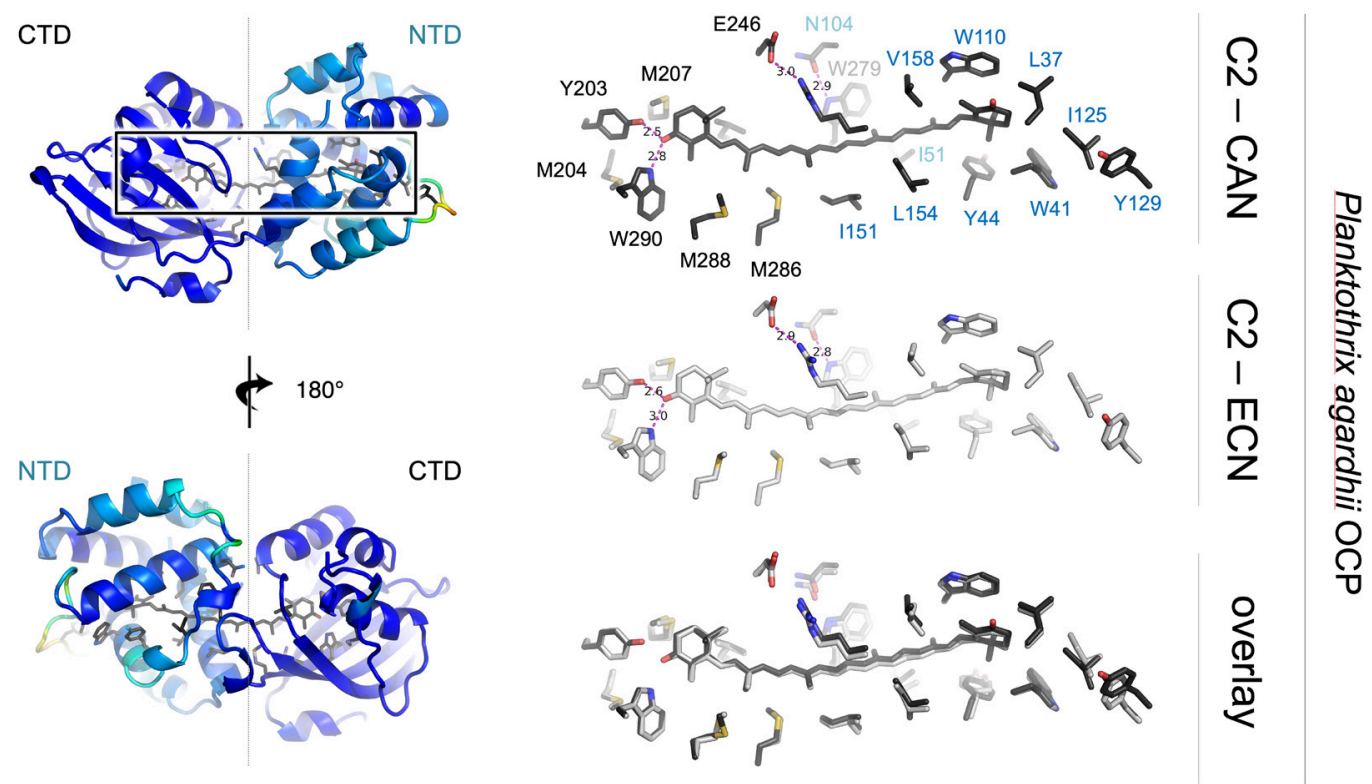

Figure 12: Conformational changes observed upon change of the functionalizing carotenoid are limited to the NTD. (A) The structure of Plankto-OCP-CAN is shown as a ribbon colored from 
cold to hot colors as a function of the RMSD to the Plankto-OCP-ECN structure. (B) Close-up view on the carotenoid and residues lining the homonymous tunnel. The orientation in $(B)$ is similar to that in the upper panel of $(A)$.

Conformational changes at the $\mathrm{C} \alpha$ level are best visualized in $\mathrm{C} \alpha-\mathrm{C} \alpha$ distance difference matrices (DDM). Calculated using the $C 2$ Ntag-Plankto-OCP $P_{C A N}$ structure as a reference, these confirm that the main difference between the $P 2{ }_{1}$ chain $A, P 2{ }_{1}$ chain $B$ and $C 2$ structures is a change in the orientation of the NTD vs. the CTD, accompanied (or triggered) by a change in the conformation of $\alpha \mathrm{A}$ and the $\alpha \mathrm{C}-\alpha \mathrm{D}$ and $\alpha \mathrm{G}-\alpha \mathrm{H}$ loops. As a result, the two loops draw away from the CTD, pulling with them $\alpha \mathrm{C}, \alpha \mathrm{D}, \alpha \mathrm{E}, \alpha \mathrm{F}$ and $\alpha \mathrm{G}$ (Figure 13A). Conformational changes are far less pronounced in the CTD, where only $\beta 5$ slightly changes position, moving away from $\alpha J, \beta 2$ and $\alpha M$ (Figure 13A). In this context, it should be recalled that aside $\alpha A$, the NTD consists of two 4-helix bundles contributed by helices $\alpha \mathrm{B}-\alpha \mathrm{C}-\alpha \mathrm{H}-\alpha \mathrm{l}$ and $\alpha \mathrm{D}-\alpha \mathrm{E}-\alpha \mathrm{F}-\alpha \mathrm{G}$, respectively, which appose one onto the other leaving a central void that constitutes the carotenoid tunnel in the NTD. Our structural comparison indicates that regions most affected by the change in space group are those linking the two bundles, with conformational changes affecting the position - but not the internal structure - of the second bundle with respect to the CTD. Interestingly, the $\alpha \mathrm{C}-\alpha \mathrm{D}$ and $\alpha \mathrm{G}-\alpha \mathrm{H}$ loops are also the secondary structure elements most affected by the change in functionalizing carotenoid. It is visible from the comparison of the $C 2$ structures, that these changes result in a modification of the distance between helices $\alpha \mathrm{D}$ and $\alpha \mathrm{F}$, on the one hand, and the CTD, on the other hand. Thus, the structural dynamics at the basis of the change in space group and those resulting from the change in functionalizing carotenoid are localized in the same regions of the protein. This suggests that they could reflect a functional role. In line with this hypothesis, calculation of a DDM between the isolated NTD of Syn-OCP ${ }_{C A N}$, considered as a surrogate for the structure of the NTD in OCP ${ }^{R}$, and the NTD in the dark-adapted Syn-OCP ${ }_{C A N}{ }^{\circ}$ reveals major changes in the $\alpha C-\alpha D$ and $\alpha G-\alpha H$ loops (Figure 13B), which result in a dramatic rearrangement of the first $\alpha B-\alpha C-\alpha H-\alpha$ l bundle whilst leaving the internal conformation of the $\alpha \mathrm{D}-\alpha \mathrm{E}-\alpha \mathrm{F}-\alpha \mathrm{G}$ bundle unperturbed. Thus, like changes in space group and functionalizing carotenoid in Ntag-Plankto-OCP, photoactivation affects the internal structure and relative positioning of the Syn-OCP $\alpha B-\alpha C-\alpha H-\alpha l$ bundle through conformational changes in the $\alpha \mathrm{C}-\alpha \mathrm{D}$ and $\alpha \mathrm{G}-\alpha \mathrm{H}$ loops, while the second $\alpha \mathrm{D}-\alpha \mathrm{E}-\alpha \mathrm{F}-\alpha \mathrm{G}$ bundle appears stable and acts as a base. This observation is surprising given that the interface between the first bundle and the CTD is larger (BSA of $643.2 \AA^{2}$; four $\mathrm{H}$-bonds and a salt bridge) than that between the second bundle and the CTD (BSA of $189.3 \AA^{2} ; 1 \mathrm{H}$-bond). Regardless, our results show that the two most mobile regions across the NTD - and therefore, across the whole Plankto-OCP - are the $\alpha \mathrm{C}-\alpha \mathrm{D}$ and $\alpha \mathrm{G}-\alpha \mathrm{H}$ loops, making these the first candidates to explain the increased photoactivation efficiency of Plankto-OCP. 
A
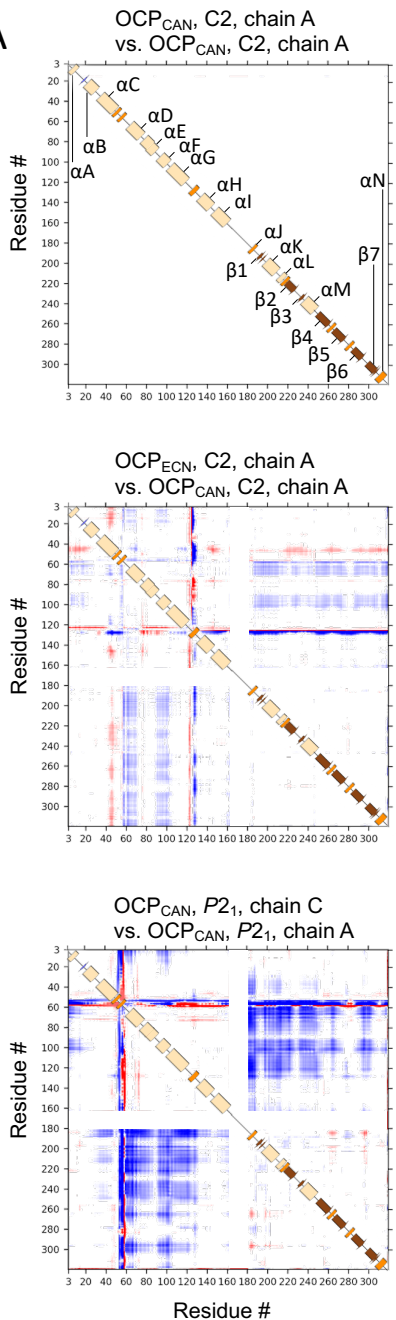
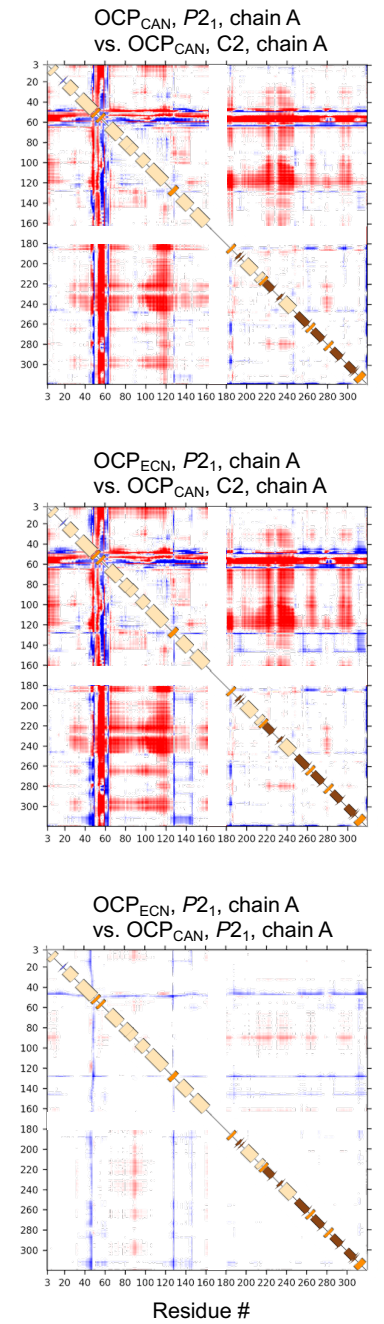

B

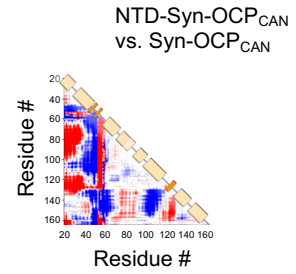

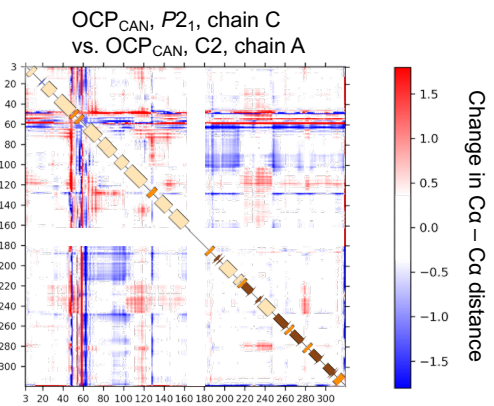
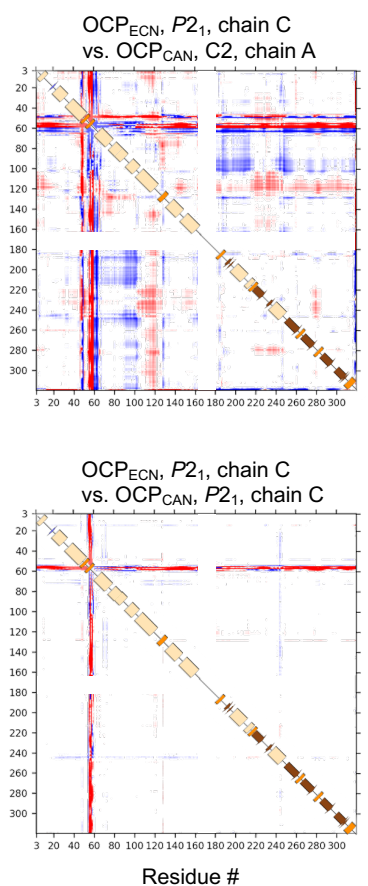

C

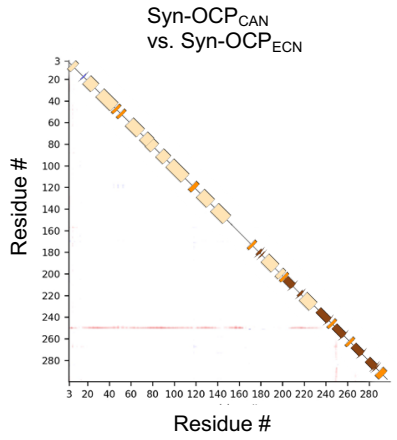

Figure 13: Crystal packing traps different conformations of the Ntag-Plankto-OCP monomers. (A) Changes in $\mathrm{Ca}-\mathrm{C} \alpha$ distances across in the various $\mathrm{ECN}-$ and $\mathrm{CAN}$-functionalized $\mathrm{C} 2$ and $P 21$ chains were monitored by computing $\mathrm{C} \alpha-\mathrm{C} \alpha$ difference-distance matrices (DDM). As a reference structure, we used the C2 Plankto-OCP-CAN (2 first rows), or either chain A or C of the $P 21$ PlanktoOCP-CAN structure (third line). In each DDM, the lower and upper panels (separated by a sketch of the secondary structure) show the changes in $\mathrm{Ca}-\mathrm{C} \alpha$ distances for alternate conformers $\mathrm{A}$ and $B$ with respect to the reference structure. Indeed, alternate conformations are seen in all PlanktoOCP structures. The overall similarity between upper and lower panels indicates that alternate conformations hardly affect the protein backbone. In the $P 2_{1}$ crystals, two chains are found, which either display a more expanded (chain A) or a more compact (chain B) structure, due to changes in the opening angle between the NTD and the CTD. The DDM further indicates that these changes 
stem from helix $\alpha \mathrm{D}$, and the $\alpha \mathrm{C}-\alpha \mathrm{D}$ and $\alpha \mathrm{G}-\alpha \mathrm{H}$ loops, either drawing away (chain $\mathrm{A}$ ) or coming closer (chain B) to the CTD, respectively. Comparison of the C2-CAN and C2-ECN structures suggests that the presence of CAN results in a more compact protein. Support to this hypothesis could not be obtained from comparison of the $P 2{ }_{1}$ chain $A$ or $P 2{ }_{1}$ chain $B$ structures. Nonetheless, we observe a similar trend when comparing, either in the CAN-functionalized or the ECN-functionalized states, $P 21$ chain $C$ and $P 21$ chain $A$. Thus, changes in functionalizing carotenoid have a lesser influence on the OCP conformation than changes in space group. (B) DDM calculated for the isolated NTD of SynOCP-CAN vs. Syn-OCP-CAN. This DDM suggests that upon photoactivation, large scale conformational changes occur in the NTD that mainly result in helix $\alpha B$ drawing away from helices $\alpha D$ to $\alpha \mathrm{G}$, while the $\alpha \mathrm{C}-\alpha \mathrm{D}$ loop comes closer to these and to $\alpha \mathrm{H}$ to $\alpha \mathrm{l}$. Also, $\alpha \mathrm{G}$ edges closer to helices $\alpha \mathrm{H}$ to $\alpha \mathrm{l}$, while the $\alpha \mathrm{G}-\alpha \mathrm{H}$ loop draws farther. (C) DDM calculated for Syn-OCP ${ }_{\text {CAN }}$ vs. Syn-OCPECN. Hardly no change in the Syn-OCP structure is seen upon change in the functionalizing carotenoid.

The $\alpha \mathrm{C}-\mathrm{\alpha D}$ loop conformation differs in the $P 2_{1}$ chain $\mathrm{A}, P 2_{1}$ chain $\mathrm{B}$ and $\mathrm{C} 2$ structures, but is overall preserved in each pair of ECN- and CAN-functionalized structures - notwithstanding the presence of multiple residues in alternate conformations in the $\mathrm{C} 2 \mathrm{Ntag}-\mathrm{Plankto-OCP_{ \textrm {ECN } }}$ structure (Figure 11 and Supplementary Table S2). The $\alpha \mathrm{G}-\alpha \mathrm{H}$ loop conformation is overall preserved among $P 2{ }_{1}$ structures, with only a slight displacement of $\alpha \mathrm{H}$ observed between the $P 2{ }_{1}$ chains $A$ and $B$, as a result of changes in the $\alpha C$ conformation concomitant to those in the $\alpha \mathrm{C}-\alpha \mathrm{D}$ loop (Figures 11 and 13). The $\alpha \mathrm{G}-\alpha \mathrm{H}$ loop conformation is yet more divergent among the $C 2$ structures, with the $C 2$ Ntag-Plankto-OCP ${ }_{E C N}$ structure displaying two alternate mainchain conformations for the highly-conserved residues 122-VAPIPSGYKL-130, of which none overlaps with the conformations observed in C2 Ntag-Plankto-OCP ${ }_{C A N}$ and in the $P 2{ }_{1}$ chains (Supplementary Figure 6 and Supplementary Table S2). Interestingly, these changes in the conformations of the $\alpha \mathrm{C}-\alpha \mathrm{D}$ and $\alpha \mathrm{G}-\alpha \mathrm{H}$ loops result in the opening/closing of water-channels from the protein surface to the carotenoid tunnel in the NTD. Briefly, four main channels (\#1, $\# 2$, \#3 and \#4) can be identified on the basis of our structures (Supplementary Figures 7, 8 and 9). The first one (\#1), which is colinear with the carotenoid axis and adjacent to the $\beta 2$ ring, features the fully conserved L37, M83, M117 and I125 (Supplementary Figures 7, 8 and 9) at its bottleneck and encompasses the binding site of the carotenoid in the structure of the isolated NTD of Syn-OCP CAN (Supplementary Tables S2 and S4). Channel \#1 is opened in all Ntag-Plankto-OCP structures, and as well in all previously determined OCP structures. In the C2 Ntag-Plankto-OCP $\mathrm{ECN}_{\mathrm{N}}$ structure, however, the presence of two alternate main-chain conformations for $\alpha \mathrm{G}-\alpha \mathrm{H}$ loop residues 120-GIAPIPSGYKL-131 (Supplementary Figure S6) results in two configurations of the channel (see Supplementary Table S4) characterized by either a wider or narrower opening (Supplementary Figures 6, 8 and 9). The enlargement of channel \#1 is compulsory for the carotenoid to translocate fully across the NTD, as illustrated by the observation that the $\alpha \mathrm{G}-\alpha \mathrm{H}$ loop displays a large change in conformation in the structure of the isolated NTD of Syn-OCP ${ }_{\text {CAN }}$, compared to the NTD in dark-adapted Syn-OCP ${ }_{\text {CAN }}$

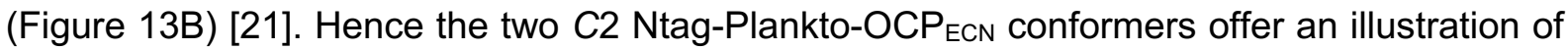


how structural dynamics in the $\alpha \mathrm{G}-\alpha \mathrm{H}$ loop may participate in the regulation of photoactivation. We yet note that none of the conformations observed in our various Ntag-Plankto-OCP structures, or in the previously determined OCP structures, matches that found in the structure of the isolated NTD of Syn-OCP $P_{\text {CAN. }}$

The second (\#2), third (\#3) and fourth channel (\#4) are all perpendicular to the carotenoid tunnel, and open on either side of Y44 side chain, ending up just above the $\beta 2$-ring of the carotenoid (\#2) or its second terpene unit (\#3, \#4) (Supplementary Figures 7 and 8). Channel \#2 features W41 and Y44 at its bottleneck, whereas channel \#3 is lined by Y44, M47, I51, I151 and F280, and channel \#4 by Y44, Y111 and aG (Supplementary Figures 7 and 8). Residues W41, M47, I151 and M280 are strictly conserved, whereas V53 and I53 fill the structural position of Plankto-OCP I51 in Ana-OCP and Syn-OCP, respectively, and Y44 and Y111 are replaced by a phenylalanine (F44) and an asparagine (N111), respectively, in Ana-OCP. We find that channel \#2 is opened toward the bulk in all $P 2{ }_{1}$ structures, albeit a much wider opening is observed in chain $A$ of the $P 2{ }_{1}$ Ntag-Plankto-OCP $P_{E C N}$ structure, due to a large swing of $Y 44$ towards Y111 (Supplementary Figure 8) - i.e. away from W41. Of note, Y44 is found in alternate side-chain conformations in the $C 2$ Ntag-Plankto-OCP $P_{C A N}$ structure, and as well in the previously determined Syn-OCP $\mathrm{ECN}_{\mathrm{E}}$ structure (Supplementary Figure 8). Additionally, channel \#3 is opened in chain A of the $P 2{ }_{1}$ Ntag-Plankto-OCP $P_{C A N}$ and Ntag-Plankto-OCP $P_{E C N}$ structures, although in only one alternate conformer due to alternate conformations in M47. In both C2 structures, channel \#2 and \#3 are closed. Nonetheless, in one of the two alternate

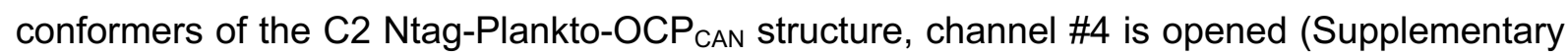
Figure 7). We note that all previously determined OCP structures feature similar openings of the carotenoid tunnel towards the bulk that are perpendicular to the carotenoid axis. For example, channels \#3 and \#4 are opened in the Syn-OCP structures (PDB ids: $3 m g 1$ and 4xb5), while channels \#2 and channel \#3 are opened in the Tolypothrix OCP structure (PDB id: $6 \mathrm{pq} 1)$ and in the two chains of the asymmetric unit dimer from the Arthrospira structure (PDB id: 5ui2; also referred to as Limnospira). In Tolypothrix OCP, a supplementary channel $(\# 5)$ is observed, $\sim 180^{\circ}$ apart from channel \#2, which is lined by the highly conserved F28, 140, S157 and M161 (Supplementary Figure 10). The opening of channel \#5, which alike channel \#2 ends up above the $\beta 2$-ring of the carotenoid albeit from the other side of the tunnel, mainly depends on the side chain conformation of M161 in the Tolypothrix OCP structure. Channel \#5 is also opened in the two chains of the asymmetric unit dimer from the Anabaena OCP structure (PDB id: $5 \mathrm{hgr}$ ), where its opening if facilitated by the substitution of 140 (in Plankto-OCP, Syn-OCP, Arthrospira and Tolypothrix OCPs) for a valine - atop the conformational change in M161. Channel \#3 is additionally opened in chain B of Ana-OCP, but closed in chain A due to change in the conformation of M47. Thus, the Ntag-Plankto-OCP 
structures illustrate that the opening of channel \#1-4 mainly depends on the conformations displayed by residues in $\alpha \mathrm{C}$ (A33-E46) and in the $\alpha \mathrm{C}-\alpha \mathrm{D}$ (M47-G57) and $\alpha \mathrm{G}-\alpha \mathrm{H}$ (G120-L131) loops. The Tolypothrix and Anabaena OCP structures additionally reveal the existence of another channel (\#5), which ends up on the side of the carotenoid $\beta 2$ ring where the methyl or carbonyl oxygen atom of ECN and CAN are exposed, respectively. Given that the Tolypothrix OCP features the same residue distribution as Plankto-OCP, Syn-OCP and Arthrospira OCP along the five channels, it is possible that channel \#5 would open in these as well.

We noted earlier that the positioning of the carotenoid differs in the various Ntag-Plankto-OCP structures, due to changes in the opening angle between domains, i.e. at the NTD/CTD interface. Besides this point, the most notable dissimilarity in the carotenoid tunnel is the presence of a methionine at position 207, instead of a leucine in other OCPs (L205 in SynOCP, and L207 in Anabaena, Arthrospira and Tolypothrix OCP) (Supplementary Figures 5, 6, 10). At position 288, a methionine is found in Plankto-OCP, Ana-OCP (PDB id: 5hgr) and Tolypothrix (PDB id: 6pq1)) OCP (PDB id: 4xb5), which is substituted for by an isoleucine in Synechocystis (1286) and Arthrospira OCP (1288) (Supplementary Figures 5, 6, 10). Thus, Plankto-OCP is particularly enriched in methionine residues. Of these two substitutions, we speculate that the one which may be related to the higher photoactivation rate of OCP is the former, since the slower Tolypothrix and Anabaena OCP also feature a methionine at position 288. Conversely, only Plankto-OCP features a methionine at position 207 . The only other structural difference between the carotenoid tunnels of Plankto-OCP and Syn-OCP is the placement of Plankto-OCP I51 at the position occupied by Syn-OCP I53, due to changes in the sequence of the $\alpha C-\alpha D$ loop (47-MGKTITVAALGAA-59 in Plankto-OCP vs. 47MGKTLTIAAPGAA-59 in Syn-OCP) which adopts the same conformation in Syn-OCP, AnaOCP, Arthro-OCP and Toply-OCP structures, but exhibits varying conformations in the various Ntag-Plankto-OCP structures (Supplementary Figures 10 and 12). Given that the $\alpha C-\alpha D$ loop in Ana-OCP (47-MGKTITVAAPGAA-59) differs from that in Plankto-OCP only by the substitution of L56 for a proline - found as well in Syn-OCP, Arthro-OCP and Toply-OCP - it is reasonable to assign the increased dynamics of this loop in Plankto-OCP to this residue. P56 indeed fits into a groove at the surface of the NTD (formed by S60, M61, G108, Y111 and W277 in Syn-OCP; S60, M61, G108, N111 and W279 in Ana-OCP; and N60, M61, G108, Y111 and W279 in Arthro-OCP and Toply-OCP), whereas it is solvent exposed in Plankto-OCP (Supplementary Figure 10). 


\section{Discussion}

Here, we report results from a comparative analysis of OCP1 functional properties across different cyanobacterial species, including the previously uncharacterized Plankto-OCP and the most studied Syn-OCP. All OCP-related functional properties - photoactivation, thermal recovery, interaction of the $\mathrm{OCP}^{\mathrm{R}}$ state with the $\mathrm{PBS}$ and consequential fluorescence quenching - were examined for their dependence on the functionalizing carotenoid (ECN vs. CAN). We also investigated the influence of His-tagging at either the $\mathrm{N}$ or $\mathrm{C}$-terminus, which afforded information on the role of the NTE $(\alpha A)$ and the CTT $(\alpha N)$ in the various molecular processes. In an attempt to rationalize functional observations on the basis of structural differences, we solved the hitherto uncharacterized structures of Plankto-OCP in the ECN- and CAN-functionalized states, each in two space groups, and compared them to the available structures of ECN- and CAN-functionalized Syn-OCP [17, 21]. When useful, we also included in our structural comparison the structures of CAN-functionalized Anabaena OCP [58], hECNfunctionalized Arthrospira OCP [8] and of CAN-functionalized Tolypothrix OCP [30].

Initially, our interest in Plankto-OCP was sparked by the recent observation that the in vivo OCP-related NPQ-mechanism is more efficient in Planktothrix than in Synechocystis cells [37]. The functional characterization of Plankto-OCP properties revealed the molecular basis for this phenotype, showing that it not only photoactivates faster than Syn-OCP, but also recovers faster (especially at $9^{\circ} \mathrm{C}$ ). Such a fast thermal recovery had thus far been observed only for OCP2 and OCPX variants, but not for members of the OCP1 clade. Indeed, Synechocystis, Arthrospira and Tolypothrix OCP1 are all characterized by a slow recovery at $9^{\circ} \mathrm{C}$. Similar to these, the recovery of Plankto-OCP can be further accelerated by addition of FRP, confirming its appartenance to the OCP1 clade. In previous studies, it was found that slow recovery and the ability to bind to FRP coincide in the OCP1 clade, as opposed to OCP2 and OCPX [16] which recover faster thermally but are unable to bind FRP. Based on sequence alignments, it was further suggested that the defining feature of the OCP1 clade is the presence of residues R229 and D262 (Synechocystis number), which are absent in OCP2 and OCPX [16]. Our data confirm that presence of R229 and D262 correlates with the ability to bind FRP, but indicate that the slow recovery rate of Synechocystis, Arthrospira and Tolypothrix OCP1 is unrelated to their presence.

The faster photoactivation and recovery of Plankto-OCP could stem from its higher protein flexibility, illustrated by the large structural diversity amongst Plankto-OCP crystalline structures. Indeed, crystal packing traps different conformations of the Ntag-Plankto-OCP monomers, which differ (i) in the conformation displayed by $\alpha \mathrm{G}$ and the $\alpha \mathrm{C}-\alpha \mathrm{D}$ and $\alpha \mathrm{G}-\alpha \mathrm{H}$ loops, (ii) in the opening angle between domains, at the NTD/CTD interface, (iii) in the opening 
angle between monomers, in the naturally-occurring dark-adapted dimer; and (iv) in the positioning of the carotenoid (Figures 9-13). The Plankto-OCP structures offer a peek into the molecular breathing motions that animate OCP, at the monomer and the dimer levels. The $P 2_{1}$ structures demonstrate that the two monomers in a dimer can adopt slightly different structures, differing in the opening angle at the NTD/CTD interface, and consequently, in the orientation of the carotenoid in the tunnel. These differences could account for the repeated observations that two types of OCP coexist in darkness in spectroscopic experiments [40]. The conformations present in the $C 2$ structures match best those displayed by chain $\mathrm{B}$ in the $P 2_{1}$ structures. Nonetheless, they are not strictly equivalent, differing not only in the opening angle at the NTD/CTD interface, but as well in the exact positioning of helices around the carotenoid and in the conformation displayed by the $\alpha \mathrm{C}-\alpha \mathrm{D}$ and $\alpha \mathrm{G}-\alpha \mathrm{H}$ loops. These two loops indeed show the highest diversity among the various structures reported herein, whereas they adopt the same conformation in Syn-OCP, Ana-OCP, Arthro-OCP and Tolypo-OCP structures.

The $P 2_{1}$ chain $A$ and $P 2_{1}$ chain $B$ structures feature a "porous wall", traversed by channels perpendicular to the carotenoid tunnel, whereas the $C 2$ structures feature a carotenoid tunnel that is insulated from the bulk, except at its extremities (Supplementary Figures 7, 8 and 9, and Supplementary Tables S2 and S4). Together, the six chains illustrate that the opening of channels \#1-4 mainly depends on the conformations displayed by residues in aC (A33-E46; channels 1-2) and in the $\alpha C-\alpha D$ (M47-G57; channels 2-4) and $\alpha \mathrm{G}-\alpha H$ (G120-L131; channel 1) loops, and question the possible role of water access to the carotenoid tunnel in the photoactivation mechanism - and/or its limitation. The enlargement of channel \#1 is compulsory for the carotenoid to translocate fully across the NTD, and accordingly, the $\alpha \mathrm{G}-\alpha \mathrm{H}$ loop displays a large change in conformation in the isolated NTD of Syn-OCP CAN $_{\text {, as compared }}$ to the NTD in dark-adapted Syn-OCP ${ }_{\text {CAN }}$ [21] (Figure 13). Two alternate conformations are observed for this loop in the $C 2$ Ntag-Plankto-OCP $\mathrm{ECN}_{\mathrm{E}}$ structure, which offer an illustration of how structural dynamics could participate in the regulation of photoactivation. We yet note that none of the conformations observed in our various Ntag-Plankto-OCP structures, or in the previously determined OCP structures, matches that found in the structure of the isolated NTD of Syn-OCP $P_{\text {CAN }}$ [21]. We also investigated if the presence of multiple alternate conformations could underlie the higher photoactivity of Plankto-OCP. Plotting these against the secondary (Figure 9A and Supplementary Table S2) or tertiary structure, we identify the $\alpha \mathrm{C}-\alpha \mathrm{D}$ (M47G57) loop, $\alpha M$ and $\beta 4$ as clusters, i.e. secondary-structure elements or epitopes more prone to alternate conformations irrespective of the space group or functionalizing carotenoid (Figure 9A). W288, at the CTD end of the carotenoid tunnel, is present in alternate conformations in all structures. Additionally, D6, N14, Q73, Q77, M117, M207, R241, R244, V258, K275 are present in alternate conformations in at three out of the six Plankto-OCP chains analyzed. In 
conclusion, we propose that the structural traits which explain the higher photoactivation rate of Plankto-OCP are the increased flexibility in the $\alpha \mathrm{C}-\alpha \mathrm{D}$ (M47-G57) and $\alpha \mathrm{G}-\alpha \mathrm{H}$ (G120-L131) loops, which results in opening of water channels to the carotenoid tunnel, and increased molecular breathing motions at the levels of the dimer, the NTD/CTD interface, and the NTD helix core. As to the increased recovery rate, we hypothesize that it originates from a decreased stabilization of the Plankto-OCP ${ }^{R}$ state. Hence, we first compared residues distribution along the carotenoid tunnel in the NTD. The only structural difference between the NTD tunnels of Plankto-OCP and Syn-OCP is the placement of Plankto-OCP I51 at the position occupied by Syn-OCP 153, due to changes in the sequence of the $\alpha \mathrm{C}-\mathrm{aD}$ loop (47MGKTITVAALGAA-59 in Plankto-OCP vs. 47-MGKTLTIAAPGAA-59 in Syn-OCP) (Supplementary Figures 5, 6 and 10). Another, more hypothetical means by which the Plankto$O C P^{R}$ state would be rendered less stable could be through a decrease in the affinity of the CTT for the empty CTD tunnel. Syn-OCP is unique among the variants discussed herein in that it features a 314-NFAR-317 sequence at its C-terminus instead of 316-NLVR-319 in the other OCP (Supplementary Figure 10). The combined substitution of a leucine for phenylalanine, and of a valine for alanine, could account for a higher affinity of the Syn-OCP CTT for the empty CTD tunnel. Reversely, the presence in Plankto-OCP of a methionine at position 288, (Planktothrix numeration) instead of an isoleucine at position 286 in Syn-OCP, could decrease the affinity of the Plankto-OCP CTT for the empty CTD tunnel.

We also further examined the effect of His-tagging at the $\mathrm{N}$ - or $\mathrm{C}$-terminus on the various functional properties of OCP (Figures 3 and 8). Indeed, both the NTE and the CTT have been proposed to play important roles in the OCP photoactivation and recovery mechanisms. We found that irrespectively of the variant, the $\mathrm{N}$-tagged protein displays the best match to the native protein, both in terms of photoactivation rate and thermal recovery rate. Native SynOCP and Plankto-OCP display the fastest photoactivation profile, indicating that increased disorder in their NTE or a weaker interaction between the NTE and CTD does not benefit photoactivation as was previously suggested [61]. Recovery was also found to be faster in native Syn-OCP and Plankto-OCP than in their His-tagged counterparts. A dramatic drop in the recovery rate was notably observed for the $\mathrm{C}$-tagged Plankto-OCP, which could stem either from a higher stability of the $O C P^{R}$ state in presence of a C-terminal His-tag or from a frustrated recovery of the $\mathrm{OCP}^{\circ}$ state due to hindered rebinding of the CTT to the CTD $\beta$-sheet. Regardless, these results point to an important role played by the CTT in the $O C P^{R}$ to OCP recovery. Little is known regarding the configuration of the CTT residues in the $O C P^{R}$ state and its role in OCP photoactivation and recovery. However, it was shown that in the Synechocystis C-terminal domain homologue (CTDH), which belongs to a family of carotenoidtransporting proteins homologous to the OCP CTD [35, 36], the CTT can exchange between 
a closed conformation, whereby it covers the empty carotenoid tunnel, and an opened conformation, similar to that observed in $\mathrm{OCP}^{\circ}$. By analogy, it was proposed that one of the last steps of OCP photoactivation is the repositioning of the CTT on the empty CTD-half of the carotenoid tunnel. Presence of a six-histidine tag in the CTT could hinder this movement, leading to slower kinetics of photoactivation and recovery. In conclusion, our results suggest that re-binding to the CTD $\beta$-sheet of the CTT, and to a lower extent of the NTE, are rate limiting steps in the thermal recovery of the $\mathrm{OCP}^{\circ}$ state, and that completion of these steps is inhibited by presence of a His-tag at their $\mathrm{C}$ or $\mathrm{N}$-terminal extremities, respectively.

The position of the His-tag also influences the PBS quenching, with again a more pronounced effect of His-tagging at the C-terminus than at the N-terminus of OCP. In the past, we proposed that the presence of a six-histidine tag upstream the NTE could destabilize the interaction with the PBS [61], yet the present data invalidate the hypothesis, demonstrating that $\mathrm{N}$-tagged and native OCP detach as swiftly from the PBS. Contrastingly, introduction of the six-histidine tag downstream the CTT results in increased PBS-fluorescence quenching and slower fluorescence recovery. This result can be rationalized by envisioning that the C-terminal histag strongly stabilizes the PBS-bound $\mathrm{OCP}^{\mathrm{R}}$ structure, possibly through interaction of the tag with PBS amino acids. A cross-species characterization of OCP/PBS complexes allowed the discovery that irrespective of His-tagging, Plankto-OCP binds stronger to Syn-PBS than SynOCP. Furthermore, Plankto-OCP binds stronger to Syn-PBS than to Plankto-PBS, echoing the previous observation that Arthrospira and Anabaena OCPs bind stronger to Syn-PBS than to Arthrospira and Anabaena PBS, respectively [42]. Recent studies on Syn-OCP have shown that besides R155, early demonstrated as compulsory for the OCP/PBS interaction [62], residues L51, P56, G57, A58, N104, I151, and N156 play important roles in the interaction [63]. Recall that in all OCP, N104 and R155 are involved in the stabilization of the $\mathrm{OCP}^{\circ}$ state by contributing $\mathrm{H}$-bonds to the NTD/CTD interface (N104(OD1) to W277(Syn)/W279 (Plankto)(NE1); R155(NH2) to E244 (Syn)/E246 (Plankto)(OE1)). In addition, in all structures but the Plankto-OCP structures, N104 contributes to the stabilization of the linker by establishing a H-bond to E174(OE1). As G57, N104, I151, R155 and N156 are structurally conserved in Plankto-OCP, one can eliminate the hypothesis that the observed differences in Syn-PBS quenching would stem from these. As to Syn-OCP L51, it is replaced by an isoleucine in the Anabaena, Limnospira and Tolypothrix proteins, nonetheless the side chain occupies the same position in all structures, fitting in a groove contributed by M47, I151, F280 and V284, at the NTD-CTD interface. In Plankto-OCP, this residue is conserved; however, due to changes in the sequence of the $\alpha C-\alpha D$ loop (47-MGKTITVAALGAA-59 in Plankto-OCP vs. 47MGKTLTIAAPGAA-59 in Syn-OCP), Plankto-OCP L51 is found at the position occupied by 153 in all previously-determined OCP structures. Syn-OCP P56 is replaced by a leucine in Plankto- 
OCP, but it is conserved in other OCPs. Hence, the difference in PBS binding-affinity and fluorescence-quenching observed between Syn-OCP and Plankto-OCP could stem from changes in the sequence and structure of the $\alpha \mathrm{C}-\alpha \mathrm{D}$ loop. Within $4 \AA$ of R155, supposedly central to the interaction, we find four additional candidate positions at which residue substitutions could explain the higher affinity of Plankto-OCP as compared to Syn-OCP: G99, T102, A103 and C157, respectively. These residues are substituted by A99, S102, P103 and A157 in Syn-OCP, and by A99, S102, P103 and S157 in Ana-OCP. Future mutagenesis work concentrated on these results could unveil the molecular basis for this unexpected crossspecies preference for the Syn-PBS. Specific to the lower affinity of Plankto-OCP for PlanktoPBS than Syn-PBS, it must be recalled that OCP binding to the PBS is very sensitive to the structural intactness of the PBS core. Although 77K fluorescence spectra of both isolated PBS suggested a perfect energy transfer from PC to the last core emitters, we cannot discard the hypothesis that a slightly different interaction between the APC trimers could be at the origin of the weaker OCP-PBS binding observed for Arthrospira and Planktothrix species-specific complexes. It was also observed that the Plankto-Lcm protein is more sensitive to proteolysis than Syn-Lcm (Supplementary Figure 3). Hence the reduced affinity of Plankto-OCP for the Plankto-PBS could originate in the partial degradation of the Lcm component.

We last examined the influence of the functionalizing carotenoid on OCP excited and intermediate states dynamics. Indeed, irrespective of the species, CAN-functionalized OCP photoactivates faster and recovers slower than ECN-functionalized OCP. Using fs-ns and ns$s$ transient absorption spectroscopy on ECN- and CAN-functionalized Plankto-OCP and Syn$\mathrm{OCP}$, we inquired the time scale(s) on which the gain in photoactivation efficiency occurs for the CAN-functionalized proteins. We observed differences in the respective yields of the $S_{1}$, ICT and $S^{*}$ states, but nearly no change in their characteristic lifetimes nor in the $P_{1}$ yield (Table 1). Hence, the difference in photoactivation rate of ECN- and CAN-functionalized OCP does not stem from changes in their excited state dynamics nor in the P1 formation quantum yield. Furthermore, the increased $\mathrm{S}^{*}$ yield observed for CAN-functionalized Plankto-OCP and Syn-OCP is not mirrored by an increase in the $\mathrm{P}_{1}$ yield, suggesting that the former is not its only precursor. Clear differences between the four tested OCPs were yet observed in the nss time scale. First, we observed for Plankto-OCP a larger drop in difference absorption at 563 $\mathrm{nm}$ on the ns- $\mu$ s time scale, which could sign for a sub-optimal translocation of ECN and CAN into the Plankto-NTD, as compared to the Syn-NTD. This hypothesis is supported by the observation that ECN-functionalized OCP forms more compact monomers and dimers, wherein migration of the carotenoid could be facilitated (Figures 12 and 13). Regardless, the most striking differences between Plankto-OCP and Syn-OCP are visible in the ns-ms time window, while those between CAN- and ECN-functionalized OCP concentrate in the ms-s 
timescale. Thus, our results suggest that both carotenoid translocation and NTE/CTT detachments are affected by the change in protein scaffold, whereas it is domain dissociation that is most affected by a change in the functionalizing carotenoid. Our data support that domain dissociation is faster and more efficient in CAN-functionalized OCPs, with no decline observed in the difference absorption at $550 \mathrm{~nm}$. Specific to Plankto-OCP ${ }_{\mathrm{CAN}}$, a faster domain separation is observed with virtually no recovery to $\mathrm{OCP}^{\circ}$ on the $\mu \mathrm{s}-\mathrm{s}$ timescale $(100 \%$ efficiency from $\mathrm{P}_{3}$ to final $O C P^{\mathrm{R}}$ ). The increased photoactivation rate of Plankto-CAN is likely grounded in this property. Thus, the differences observed in the photoactivation speed of CANand ECN-functionalized Plankto-OCP and Syn-OCP stem from changes in the (comparativelyslow) carotenoid translocation, NTE/CTT detachment and domain dissociation steps - rather than from not changes in the excited-state dynamics or P1 formation quantum yield.

It remains unclear whether or not canthaxanthin can be generally used as a functionalizing carotenoid for OCP in the natural context. When expressed in their parent strain, Arthrospira and Synechocystis OCPs [8, 11] bind hECN, while Tolypothrix OCP1 binds CAN [30]. Recombinant expression of Arthrospira and Anabaena OCPs in Synechocystis cells also yield an hECN-functionalized proteins [32, 42]. Yet, when overexpressed and isolated from CANproducing E. coli cells, all these OCPs bind CAN, suggesting that OCPs may alternatively bind $\mathrm{hECN}$ or CAN depending on the carotenoid presents in the cells. We earlier reported the partial inability of Anabaena and Tolypothrix OCP to fully convert to $\mathrm{OCP}^{\mathrm{R}}$, when functionalized by ECN $[8,11]$. Here, thermal recovery kinetics were found to be slower in the CAN-functionalized Syn- and Plankto-OCPs, possibly due to a reduced stabilization of the $O C P^{R}$ state by ECN, as compared to CAN. Assuming that the $\beta$-rings of the carotenoid are exposed to the bulk in the $O C P^{R}$ state, as suggested by the structure of the CAN-functionalized isolated NTD of SynOCP (PDB id: 4xb4; [21]), the higher stability of the CAN-functionalized $O C P^{R}$ could result from the presence of an oxygen on its $\beta 2$ ring, favoring interaction with the bulk. A similar stability would thus be expected for hECN, which features a hydroxyl group in the $\beta 2$-ring. Hence, the possibility remains that all OCPs bind $\mathrm{hECN}$ in their parent strain. Supporting this hypothesis is the observation that quenching by Plankto-OCP of the Plankto-PBS fluorescence is efficient only when ECN is used as the functionalizing carotenoid (or when the protein is tagged at the C-terminus). This result incidentally suggests that in the Planktothrix cells, the carotenoid functionalizing OCP is not CAN.

It is difficult to rationalize the observation that CAN-OCP interaction with the PBS is weaker than that with ECN-OCP (Figures 7 and 8, and Supplementary Figure 11). These results are at variance with the observation that $C A N$ stabilizes the $O C P^{R}$ state, and suggest that the isolated $O \mathrm{OCP}^{\mathrm{R}}$ and PBS-bound-OCPR $\mathrm{P}^{\mathrm{R}}$ structures could differ. The sole difference between 
$\mathrm{CAN}$ - and $\mathrm{ECN}$-functionalized $O C \mathrm{P}^{\mathrm{R}}$ is the presence of a carbonyl oxygen on the $\beta 2$-ring, but this difference should not affect binding to the PBS, since its epitope has been mapped at the opposite end of the carotenoid tunnel in the NTD, proximate to R155. Thus, it is presumably the $\beta 1$-ring, identical in CAN and ECN, which will be in contact with the PBS. A possible explanation could be that upon OCP binding to PBS, the carotenoid migrates into to the PBS, enabling a better interaction with the bilin pigments, as proposed earlier $[21,64]$. If it is the $\beta 1$ ring that plunges into the PBS - i.e., the carotenoid moves backwards with respect to the $\mathrm{OCP}^{\circ}$ to $O C P^{R}$ transition - then the $\beta 2$-ring will be repositioned inside the highly hydrophobic carotenoid tunnel, possibly past its original position in the $\mathrm{OCP}^{\circ}$ structure, which would explain the reduced stability of the CAN-functionalized OCP-PBS complexes, due to replacement of a methyl in ECN by a carbonyl oxygen in CAN. Thus, our results support the hypothesis that the isolated and PBS-bound $O \mathrm{OCP}^{\mathrm{R}}$ differ. If true, OCP-related quenching of PBS-fluorescence would be a two-step reaction (at the very least), with first the binding of $O C P^{R}$ to $P B S$, and then a change in the $O C P^{R}$ structure - as initially proposed by [21, 64]. 


\section{Conclusion:}

We here have reported on a comparative structure-function study on two OCPs from the OCP1 clade, i.e., Syn-OCP and Plankto-OCP. Our results suggest that Plankto-OCP is more flexible and we speculate that this increase in flexibility explains its faster photoactivation and recovery. Specifically, our Plankto-OCP structures evidence increased structural dynamics in the $\alpha \mathrm{C}-\mathrm{\alpha D}$ loop, which was shown to have a central role in the interaction between $O C P^{R}$ and PBS [6264]. Increased dynamics in this loop could be at the origin of the stronger binding of PlanktoOCP (compared to Syn-OCP) to Syn-PBS. Irrespectively, our data point to more efficient carotenoid translocation and NTE/CTT detachments in Plankto-OCP. We also show that presence of a His-tag influences both the photocycle of OCP (see also Nizinski et al., submitted; doi.org/10.1101/2021.12.26.474187) and its interaction with the PBS. Most impacting is introduction of the tag at the C-terminus, which results in both large damping of the photoactivation and recovery and in stronger binding to the PBS, suggesting an important role for the CTT in these molecular processes, The CTT has been proposed to shift from open position in $\mathrm{OCP}^{\circ}$ (in which the CTT interacts with the CTD) to a close position in $O C P^{R}$ (in which the CTT presumably binds in the carotenoid tunnel); our results are also suggestive of an involvement of the CTT in PBS binding. Our work last uncovers the strong influence of the nature of the functionalizing carotenoid on all aspects of OCP function. The mere substitution on ring $\beta 2$ of a dimethyl in ECN by a carbonyl in CAN results in increased photoactivation efficiency, which our ns-s spectroscopic data suggest to be due to faster domain dissociation. Nonetheless, CAN-functionalized OCP display reduced recovery, reduced binding to PBS and reduced energy-quenching activity. These features could result from thwarted back-migration of CAN, compared to ECN, as required for recovery and, presumably, PBS quenching and stabilization of the OCP-PBS complex. 
Table 1: Lifetime, time constants and quantum yields obtained from femtosecond and nanosecond transient absorption experiments. (A) Lifetimes of ICT/S $1 / S^{*} / P_{1}$ states derived from femtosecond transient absorption measurements (from data in Figure 5 and Figure S2), and estimated formation quantum yields derived from preexponential factors at $490 \mathrm{~nm}$ (the magnitude of the bleaching band of each DAS was extracted from the fitting procedure; for more details, see the Materials and Methods section and Nizinski et al., submitted; doi.org/10.1101/2021.12.26.474187). The standard error is $10 \%$. $\mathrm{P}_{1}$ yields are corrected for its positive absorbance contribution at $500 \mathrm{~nm}$. (B) Time constants and yields (percentages in brackets) derived from ns-s transient absorption measurements for $\mathrm{P}_{1}, \mathrm{P}_{2} / \mathrm{P}_{2}$, and $\mathrm{P}_{3} / \mathrm{P}_{\mathrm{N}-\mathrm{M}-\mathrm{X}}$. Only in the case of $P_{1}$ are the value in brackets actual quantum yields; for $P_{2} / P_{2}$ and $P_{3} / P_{N-M-}$ $x$, they rather indicate the relative contribution of the corresponding exponential terms to the overall decay and growing components (indicated in bold). Percentages in the OCP ${ }^{\mathrm{R}}$ column are formation quantum yields of the red absorbing intermediates remaining at $10 \mathrm{~ms}$ (determined by a comparative actinometry method with ruthenium complex; for details, see Material and Methods section and [45]).

\begin{tabular}{|c|c|c|c|c|}
\hline Table 1A & ICT & $\mathbf{S}_{1}$ & $\mathbf{S}^{*}$ & $\overline{\mathbf{P}_{1}}$ \\
\hline Syn-OCP ${ }_{\text {CAN }}$ & 0.50 ps (25 \%) & 3.2 ps (59 \%) & 7.6 ps (16 \%) & $>1 \mathrm{~ns}(0.4 \%)$ \\
\hline Syn-OCP $\mathrm{ECN}_{\mathrm{E}}$ & 0.66 ps (36 \%) & 2.8 ps (56 \%) & 10.1 ps (8 \%) & $>1 \mathrm{~ns}(0.6 \%)$ \\
\hline Plankto-OCP CAN $_{\text {. }}$ & 0.43 ps (28 \%) & 2.8 ps (56 \%) & 7.1 ps (16 \%) & $>1 \mathrm{~ns}(0.4 \%)$ \\
\hline Plankto-OCP ${ }_{E C N}$ & 0.30 ps $(44 \%)$ & $1.7 \mathrm{ps}(40 \%)$ & 5.4 ps (16 \%) & $>1 \mathrm{~ns}(0.4 \%)$ \\
\hline Table 1B & $P_{1}$ & $P_{2} / P_{2}$ & $P_{3} / P_{N-M-X}$ & $O C P^{R}$ \\
\hline Syn-OCP ${ }_{\text {CAN }}$ & 45 ns (60 \%) & $\begin{array}{r}830 \text { ns }(15 \%) \\
22 \text { us }(6 \%)\end{array}$ & $380 \mu \mathrm{s}(19 \%)$ & $0.11 \%$ \\
\hline Syn-OCP ${ }_{E C N}$ & 46 ns (48 \%) & 400 ns (14 \%) & $\begin{array}{c}890 \mu \mathrm{\mu s}(22 \%) \\
83 \mathrm{~ms}(16 \%)\end{array}$ & $0.08 \%$ \\
\hline Plankto-OCP CAN & 52 ns (65 \%) & $1.3 \mu \mathrm{s}(35 \%)$ & $\begin{array}{c}1.1 \mathrm{~ms}(33 \%) \\
24 \mathrm{~ms}(67 \%)\end{array}$ & $0.17 \%$ \\
\hline Plankto-OCP $\mathrm{ECN}_{\mathrm{N}}$ & 41 ns (35 \%) & $\begin{array}{r}410 \text { ns (31 \%) } \\
1.9 \mu \mathrm{s}(19 \%)\end{array}$ & $3.0 \mathrm{~ms}(15 \%)$ & $0.05 \%$ \\
\hline
\end{tabular}


Table 2. Data collection and refinement statistics. Statistics for the highest-resolution shell are shown in parentheses.

\begin{tabular}{|c|c|c|c|c|}
\hline & C2_100K_ECN & C2_100K_CAN & P21_100K_ECN & P21_100K_CAN \\
\hline PDB id. & 7QD0 & $\overline{7} Q C Z$ & 7QD1 & 7QD2 \\
\hline Wavelength & 0.95 & 1.54 & 0.98 & 0.99 \\
\hline Resolution range & $\begin{array}{r}32.44-1.7(1.76- \\
1.7)\end{array}$ & $\begin{array}{r}18.26-1.85(1.92- \\
1.85)\end{array}$ & $\begin{array}{r}75.58-1.71(1.75- \\
1.71)\end{array}$ & $\begin{array}{r}43.6-1.4(1.45- \\
1.4)\end{array}$ \\
\hline Space group & $C 121$ & C121 & $P 1211$ & $P 1211$ \\
\hline Unit cell & $\begin{array}{r}81.464 .8761 .69 \\
90117.91190\end{array}$ & $\begin{array}{r}82.0167 .561 .94 \\
90117.82190\end{array}$ & $\begin{array}{r}63.3665 .9077 .37 \\
90102.35490\end{array}$ & $\begin{array}{r}63.4665 .9477 .28 \\
90102.26290\end{array}$ \\
\hline Total reflections & $100512(10488)$ & $57189(4493)$ & 214925 (6579) & $272526(27565)$ \\
\hline Unique reflections & $30920(3104)$ & $23822(1963)$ & $60669(2735)$ & $115206(11285)$ \\
\hline Multiplicity & $3.3(3.4)$ & $2.4(2.3)$ & $3.5(2.4)$ & $2.4(2.4)$ \\
\hline Completeness (\%) & $98.61(99.42)$ & $92.59(76.47)$ & $90.0(54.8)$ & $93.90(92.54)$ \\
\hline Mean I/sigma(I) & $13.78(1.84)$ & $11.96(1.81)$ & $11.16(1.21)$ & $11.47(1.90)$ \\
\hline Wilson B-factor & 25.57 & 26.48 & 27.75 & 21.13 \\
\hline R-merge & $0.046(0.6095)$ & $0.052(0.5806)$ & $0.063(0.685)$ & $0.036(0.3938)$ \\
\hline R-meas & $0.055(0.7256)$ & $0.066(0.7384)$ & $0.074(0.877)$ & $0.046(0.5066)$ \\
\hline R-pim & $0.030(0.3894)$ & $0.040(0.4513)$ & $0.038(0.547)$ & $0.028(0.3153)$ \\
\hline CC1/2 & $0.999(0.843)$ & $0.997(0.682)$ & $0.997(0.426)$ & $0.998(0.852)$ \\
\hline $\begin{array}{l}\text { Reflections used in } \\
\text { refinement }\end{array}$ & 30858 (3097) & 23803 (1963) & 60658 (1519) & $115047(11285)$ \\
\hline $\begin{array}{l}\text { Reflections used } \\
\text { for R-free }\end{array}$ & $1545(154)$ & $1190(98)$ & $3015(81)$ & $5753(564)$ \\
\hline R-work & $0.1785(0.3045)$ & $0.1761(0.3740)$ & $0.1806(0.4771)$ & $0.2002(0.2804)$ \\
\hline R-free & $0.2231(0.3397)$ & $0.2450(0.4107)$ & $0.2159(0.5757)$ & $0.2309(0.2942)$ \\
\hline CC(work) & $0.967(0.901)$ & $0.968(0.771)$ & $0.942(0.671)$ & $0.956(0.626)$ \\
\hline CC(free) & $0.957(0.811)$ & $0.937(0.709)$ & $0.923(0.470)$ & $0.942(0.563)$ \\
\hline $\begin{array}{l}\text { Number of non- } \\
\text { hydrogen atoms }\end{array}$ & 3042 & 3017 & 5885 & 5819 \\
\hline macromolecules & 2698 & 2542 & 5099 & 5120 \\
\hline ligands & 85 & 42 & 82 & 100 \\
\hline solvent & 259 & 433 & 704 & 599 \\
\hline Protein residues & 308 & 309 & 621 & 614 \\
\hline RMS(bonds) & 0.030 & 0.005 & 0.05 & 0.007 \\
\hline RMS(angles) & 1.44 & 0.86 & 1.34 & 0.94 \\
\hline $\begin{array}{l}\text { Ramachandran } \\
\text { favored (\%) }\end{array}$ & 98.36 & 97.70 & 98.86 & 98.68 \\
\hline $\begin{array}{l}\text { Ramachandran } \\
\text { allowed (\%) }\end{array}$ & 0.66 & 1.64 & 0.98 & 0.99 \\
\hline $\begin{array}{l}\text { Ramachandran } \\
\text { outliers (\%) }\end{array}$ & 0.99 & 0.66 & 0.16 & 0.33 \\
\hline $\begin{array}{l}\text { Rotamer outliers } \\
\text { (\%) }\end{array}$ & 3.44 & 2.91 & 2.76 & 2.18 \\
\hline Molprobity score & 1.84 & 1.79 & 1.72 & 1.51 \\
\hline Clashscore & 7.46 & 6.65 & 5.68 & 5.02 \\
\hline Average B-factor & 34.30 & 30.31 & 32.37 & 30.40 \\
\hline macromolecules & 32.93 & 28.56 & 31.03 & 29.61 \\
\hline ligands & 37.47 & 23.55 & 24.54 & 24.16 \\
\hline solvent & 47.50 & 41.26 & 43.00 & 38.15 \\
\hline
\end{tabular}




\section{References:}

[1] N. Adir, H. Zer, S. Shochat, I. Ohad, Photoinhibition - a historical perspective, Photosynth Res, 76 (2003) 343-370.

[2] A. Krieger-Liszkay, Singlet oxygen production in photosynthesis, Journal of Experimental Botany, 56 (2005) 337-346.

[3] K.K. Niyogi, T.B. Truong, Evolution of flexible non-photochemical quenching mechanisms that regulate light harvesting in oxygenic photosynthesis, Current opinion in plant biology, 16 (2013) 307-314.

[4] D. Kirilovsky, C.A. Kerfeld, The orange carotenoid protein in photoprotection of photosystem II in cyanobacteria, Biochim Biophys Acta, 1817 (2012) 158-166.

[5] D. Kirilovsky, C.A. Kerfeld, Cyanobacterial photoprotection by the orange carotenoid protein, Nature plants, 2 (2016) 16180.

[6] F. Muzzopappa, D. Kirilovsky, Changing Color for Photoprotection: The Orange Carotenoid Protein, Trends Plant Sci, (2019).

[7] A. Wilson, G. Ajlani, J.M. Verbavatz, I. Vass, C.A. Kerfeld, D. Kirilovsky, A soluble carotenoid protein involved in phycobilisome-related energy dissipation in cyanobacteria, Plant Cell, 18 (2006) 992-1007.

[8] C.A. Kerfeld, M.R. Sawaya, V. Brahmandam, D. Cascio, K.K. Ho, C.C. Trevithick-Sutton, D.W. Krogmann, T.O. Yeates, The crystal structure of a cyanobacterial water-soluble carotenoid binding protein, Structure, 11 (2003) 55-65.

[9] A. Sedoud, R. Lopez-Igual, A. Ur Rehman, A. Wilson, F. Perreau, C. Boulay, I. Vass, A. Krieger-Liszkay, D. Kirilovsky, The Cyanobacterial Photoactive Orange Carotenoid Protein Is an Excellent Singlet Oxygen Quencher, Plant Cell, 26 (2014) 1781-1791.

[10] M. Gwizdala, A. Wilson, D. Kirilovsky, In vitro reconstitution of the cyanobacterial photoprotective mechanism mediated by the Orange Carotenoid Protein in Synechocystis PCC 6803, Plant Cell, 23 (2011) 2631-2643.

[11] A. Wilson, C. Punginelli, A. Gall, C. Bonetti, M. Alexandre, J.M. Routaboul, C.A. Kerfeld, R. van Grondelle, B. Robert, J.T. Kennis, D. Kirilovsky, A photoactive carotenoid protein acting as light intensity sensor, Proc. Natl. Acad. Sci. U. S. A., 105 (2008) 12075-12080.

[12] E.G. Maksimov, N.N. Sluchanko, Y.B. Slonimskiy, E.A. Slutskaya, A.V. Stepanov, A.M. Argentova-Stevens, E.A. Shirshin, G.V. Tsoraev, K.E. Klementiev, O.V. Slatinskaya, E.P. Lukashev, T. Friedrich, V.Z. Paschenko, A.B. Rubin, The photocycle of orange carotenoid protein conceals distinct intermediates and asynchronous changes in the carotenoid and protein components, Scientific reports, 7 (2017) 15548.

[13] M.Y. Gorbunov, F.I. Kuzminov, V.V. Fadeev, J.D. Kim, P.G. Falkowski, A kinetic model of non-photochemical quenching in cyanobacteria, Biochim Biophys Acta, 1807 (2011) 15911599.

[14] H. Bao, M.R. Melnicki, E.G. Pawlowski, M. Sutter, M. Agostoni, S. Lechno-Yossef, F. Cai, B.L. Montgomery, C.A. Kerfeld, Additional families of orange carotenoid proteins in the photoprotective system of cyanobacteria, Nature plants, 3 (2017) 17089. 
[15] S. Lechno-Yossef, M.R. Melnicki, H. Bao, B.L. Montgomery, C.A. Kerfeld, Synthetic OCP heterodimers are photoactive and recapitulate the fusion of two primitive carotenoproteins in the evolution of cyanobacterial photoprotection, Plant J, 91 (2017) 646-656.

[16] F. Muzzopappa, A. Wilson, D. Kirilovsky, Interdomain interactions reveal the molecular evolution of the orange carotenoid protein, Nature plants, 5 (2019) 1076-1086.

[17] A. Wilson, J.N. Kinney, P.H. Zwart, C. Punginelli, S. D'Haene, F. Perreau, M.G. Klein, D. Kirilovsky, C.A. Kerfeld, Structural determinants underlying photoprotection in the photoactive orange carotenoid protein of cyanobacteria, J Biol Chem, 285 (2010) 18364-18375.

[18] S. Gupta, M. Sutter, S.G. Remesh, M.A. Dominguez-Martin, H. Bao, X.A. Feng, L.G. Chan, C.J. Petzold, C.A. Kerfeld, C.Y. Ralston, X-ray radiolytic labeling reveals the molecular basis of orange carotenoid protein photoprotection and its interactions with fluorescence recovery protein, J Biol Chem, 294 (2019) 8848-8860.

[19] P.E. Konold, I.H.M. van Stokkum, F. Muzzopappa, A. Wilson, M.L. Groot, D. Kirilovsky, J.T.M. Kennis, Photoactivation Mechanism, Timing of Protein Secondary Structure Dynamics and Carotenoid Translocation in the Orange Carotenoid Protein, Journal of the American Chemical Society, 141 (2019) 520-530.

[20] Y.B. Slonimskiy, E.G. Maksimov, E.P. Lukashev, M. Moldenhauer, T. Friedrich, N.N. Sluchanko, Engineering the photoactive orange carotenoid protein with redox-controllable structural dynamics and photoprotective function, Biochimica et biophysica acta. Bioenergetics, 1861 (2020) 148174.

[21] R.L. Leverenz, M. Sutter, A. Wilson, S. Gupta, A. Thurotte, C. Bourcier de Carbon, C.J. Petzold, C. Ralston, F. Perreau, D. Kirilovsky, C.A. Kerfeld, PHOTOSYNTHESIS. A 12 A carotenoid translocation in a photoswitch associated with cyanobacterial photoprotection, Science, 348 (2015) 1463-1466.

[22] M. Bondanza, L. Cupellini, P. Faccioli, B. Mennucci, Molecular Mechanisms of Activation in the Orange Carotenoid Protein Revealed by Molecular Dynamics, Journal of the American Chemical Society, 142 (2020) 21829-21841.

[23] E.G. Maksimov, E.A. Protasova, G.V. Tsoraev, I.A. Yaroshevich, A.I. Maydykovskiy, E.A. Shirshin, T.S. Gostev, A. Jelzow, M. Moldenhauer, Y.B. Slonimskiy, N.N. Sluchanko, T. Friedrich, Probing of carotenoid-tryptophan hydrogen bonding dynamics in the singletryptophan photoactive Orange Carotenoid Protein, Scientific reports, 10 (2020) 11729.

[24] N.B. Pigni, K.L. Clark, W.F. Beck, J.A. Gascon, Spectral Signatures of Canthaxanthin Translocation in the Orange Carotenoid Protein, The journal of physical chemistry, 124 (2020) 11387-11395.

[25] R. Berera, I.H. van Stokkum, S. d'Haene, J.T. Kennis, R. van Grondelle, J.P. Dekker, A mechanism of energy dissipation in cyanobacteria, Biophysical journal, 96 (2009) 2261-2267.

[26] R. Berera, I.H. van Stokkum, M. Gwizdala, A. Wilson, D. Kirilovsky, R. van Grondelle, The photophysics of the orange carotenoid protein, a light-powered molecular switch, The journal of physical chemistry, 116 (2012) 2568-2574. 
[27] P.O. Andersson, T. Gillbro, Photophysics and dynamics of the lowest excited singlet state in long substituted polyenes with implications to the very longchain limit, J. Chem. Phys, 103 (1995) 2509-2519.

[28] C.C. Gradinaru, J.T. Kennis, E. Papagiannakis, I.H. van Stokkum, R.J. Cogdell, G.R. Fleming, R.A. Niederman, R. van Grondelle, An unusual pathway of excitation energy deactivation in carotenoids: singlet-to-triplet conversion on an ultrafast timescale in a photosynthetic antenna, Proc Natl Acad Sci U S A, 98 (2001) 2364-2369.

[29] V. Balevicius, Jr., D. Abramavicius, T. Polivka, A. Galestian Pour, J. Hauer, A Unified Picture of $S^{*}$ in Carotenoids, The journal of physical chemistry letters, 7 (2016) 3347-3352.

[30] V. Kuznetsova, M.A. Dominguez-Martin, H. Bao, S. Gupta, M. Sutter, M. Kloz, M. Rebarz, M. Precek, Y. Chen, C.J. Petzold, C.Y. Ralston, C.A. Kerfeld, T. Polivka, Comparative ultrafast spectroscopy and structural analysis of OCP1 and OCP2 from Tolypothrix, Biochimica et biophysica acta. Bioenergetics, 1861 (2020) 148120.

[31] T. Lenzer, F. Ehlers, M. Scholz, R. Oswald, K. Oum, Assignment of carotene $\mathrm{S}^{*}$ state features to the vibrationally hot ground electronic state, Phys Chem Chem Phys, 12 (2010) 8832-8839.

[32] C. Bourcier de Carbon, A. Thurotte, A. Wilson, F. Perreau, D. Kirilovsky, Biosynthesis of soluble carotenoid holoproteins in Escherichia coli, Scientific reports, 5 (2015) 9085.

[33] S. Gupta, M. Guttman, R.L. Leverenz, K. Zhumadilova, E.G. Pawlowski, C.J. Petzold, K.K. Lee, C.Y. Ralston, C.A. Kerfeld, Local and global structural drivers for the photoactivation of the orange carotenoid protein, Proc Natl Acad Sci U S A, 112 (2015) E5567-5574.

[34] A. Mezzetti, M. Alexandre, A. Thurotte, A. Wilson, M. Gwizdala, D. Kirilovsky, Two-Step Structural Changes in Orange Carotenoid Protein Photoactivation Revealed by Time-Resolved Fourier Transform Infrared Spectroscopy, The journal of physical chemistry, 123 (2019) 32593266.

[35] D. Harris, F. Muzzopappa, F. Glaser, A. Wilson, D. Kirilovsky, N. Adir, Structural dynamics in the $\mathrm{C}$ terminal domain homolog of orange carotenoid Protein reveals residues critical for carotenoid uptake, Biochimica et biophysica acta. Bioenergetics, 1861 (2020) 148214.

[36] D. Harris, A. Wilson, F. Muzzopappa, N.N. Sluchanko, T. Friedrich, E.G. Maksimov, D. Kirilovsky, N. Adir, Structural rearrangements in the C-terminal domain homolog of Orange Carotenoid Protein are crucial for carotenoid transfer, Communications Biology, 1 (2018) 125.

[37] C. Djediat, K. Feilke, A. Brochard, L. Caramelle, S. Kim Tiam, P. Setif, T. Gauvrit, C. Yepremian, A. Wilson, L. Talbot, B. Marie, D. Kirilovsky, C. Bernard, Light stress in green and red Planktothrix strains: The orange carotenoid protein and its related photoprotective mechanism, Biochimica et biophysica acta. Bioenergetics, 1861 (2020) 148037.

[38] A. Wilson, C. Punginelli, M. Couturier, F. Perrau, D. Kirilovsky, Essential role of two tyrosines and two tryptophans on photoprotection activity of the Orange Carotenoid Protein, Biochim Biophys Acta, 1807 (2011) 293-301.

[39] C. Punginelli, A. Wilson, J.M. Routaboul, D. Kirilovsky, Influence of zeaxanthin and echinenone binding on the activity of the Orange Carotenoid Protein, Biochim Biophys Acta, 1787 (2009) 280-288. 
[40] V. Slouf, V. Kuznetsova, M. Fuciman, C.B. de Carbon, A. Wilson, D. Kirilovsky, T. Polivka, Ultrafast spectroscopy tracks carotenoid configurations in the orange and red carotenoid proteins from cyanobacteria, Photosynth Res, 131 (2017) 105-117.

[41] N.N. Sluchanko, K.E. Klementiev, E.A. Shirshin, G.V. Tsoraev, T. Friedrich, E.G. Maksimov, The purple Trp288Ala mutant of Synechocystis OCP persistently quenches phycobilisome fluorescence and tightly interacts with FRP, Biochim Biophys Acta, 1858 (2017) $1-11$.

[42] D. Jallet, A. Thurotte, R.L. Leverenz, F. Perreau, C.A. Kerfeld, D. Kirilovsky, Specificity of the cyanobacterial orange carotenoid protein: influences of orange carotenoid protein and phycobilisome structures, Plant Physiol, 164 (2014) 790-804.

[43] Y. Kashino, H. Koike, K. Satoh, An improved sodium dodecyl sulfate-polyacrylamide gel electrophoresis system for the analysis of membrane protein complexes, Electrophoresis, 22 (2001) 1004-1007.

[44] G. Burdzinski, M. Bayda, G.L. Hug, M. Majchrzak, B. Marciniec, B. Marciniak, Timeresolved studies on the photoisomerization of a phenylene-silylene-vinylene type compound in its first singlet excited state, Journal of Luminescence, 131 (2011) 577-580.

[45] P. Muller, K. Brettel, [Ru(bpy)(3)](2+) as a reference in transient absorption spectroscopy: differential absorption coefficients for formation of the long-lived (3)MLCT excited state, Photochem Photobiol Sci, 11 (2012) 632-636.

[46] W. Kabsch, Xds, Acta Crystallogr D Biol Crystallogr, 66 (2010) 125-132.

[47] W. Kabsch, Integration, scaling, space-group assignment and post-refinement, Acta Crystallogr D Biol Crystallogr, 66 (2010) 133-144.

[48] A.J. McCoy, R.W. Grosse-Kunstleve, P.D. Adams, M.D. Winn, L.C. Storoni, R.J. Read, Phaser crystallographic software, Journal of applied crystallography, 40 (2007) 658-674.

[49] M.D. Winn, C.C. Ballard, K.D. Cowtan, E.J. Dodson, P. Emsley, P.R. Evans, R.M. Keegan, E.B. Krissinel, A.G. Leslie, A. McCoy, S.J. McNicholas, G.N. Murshudov, N.S. Pannu, E.A. Potterton, H.R. Powell, R.J. Read, A. Vagin, K.S. Wilson, Overview of the CCP4 suite and current developments, Acta Crystallogr D Biol Crystallogr, 67 (2011) 235-242.

[50] K. Cowtan, Fitting molecular fragments into electron density, Acta Crystallogr D Biol Crystallogr, 64 (2008) 83-89.

[51] K. Cowtan, Recent developments in classical density modification, Acta Crystallogr D Biol Crystallogr, 66 (2010) 470-478.

[52] G.N. Murshudov, P. Skubak, A.A. Lebedev, N.S. Pannu, R.A. Steiner, R.A. Nicholls, M.D. Winn, F. Long, A.A. Vagin, REFMAC5 for the refinement of macromolecular crystal structures, Acta Crystallogr D Biol Crystallogr, 67 (2011) 355-367.

[53] P. Emsley, B. Lohkamp, W.G. Scott, K. Cowtan, Features and development of Coot, Acta Crystallogr D Biol Crystallogr, 66 (2010) 486-501.

[54] W. Wriggers, K. Schulten, Protein domain movements: detection of rigid domains and visualization of hinges in comparisons of atomic coordinates, Proteins, 29 (1997) 1-14. 
[55] E. Chovancova, A. Pavelka, P. Benes, O. Strnad, J. Brezovsky, B. Kozlikova, A. Gora, V. Sustr, M. Klvana, P. Medek, L. Biedermannova, J. Sochor, J. Damborsky, CAVER 3.0: a tool for the analysis of transport pathways in dynamic protein structures, PLoS computational biology, 8 (2012) e1002708.

[56] A.A. Arteni, G. Ajlani, E.J. Boekema, Structural organisation of phycobilisomes from Synechocystis sp. strain PCC6803 and their interaction with the membrane, Biochim Biophys Acta, 1787 (2009) 272-279.

[57] S. Bandara, Z. Ren, L. Lu, X. Zeng, H. Shin, K.H. Zhao, X. Yang, Photoactivation mechanism of a carotenoid-based photoreceptor, Proc Natl Acad Sci U S A, 114 (2017) 62866291.

[58] R. Lopez-Igual, A. Wilson, R.L. Leverenz, M.R. Melnicki, C. Bourcier de Carbon, M. Sutter, A. Turmo, F. Perreau, C.A. Kerfeld, D. Kirilovsky, Different Functions of the Paralogs to the NTerminal Domain of the Orange Carotenoid Protein in the Cyanobacterium Anabaena sp. PCC 7120, Plant Physiol, 171 (2016) 1852-1866.

[59] I.A. Yaroshevich, E.G. Maksimov, N.N. Sluchanko, D.V. Zlenko, A.V. Stepanov, E.A. Slutskaya, Y.B. Slonimskiy, V.S. Botnarevskii, A. Remeeva, I. Gushchin, K. Kovalev, V.I. Gordeliy, I.V. Shelaev, F.E. Gostev, D. Khakhulin, V.V. Poddubnyy, T.S. Gostev, D.A. Cherepanov, T. Polivka, M. Kloz, T. Friedrich, V.Z. Paschenko, V.A. Nadtochenko, A.B. Rubin, M.P. Kirpichnikov, Role of hydrogen bond alternation and charge transfer states in photoactivation of the Orange Carotenoid Protein, Commun Biol, 4 (2021) 539.

[60] M. Golub, M. Moldenhauer, F.J. Schmitt, A. Feoktystov, H. Mandar, E. Maksimov, T. Friedrich, J. Pieper, Solution Structure and Conformational Flexibility in the Active State of the Orange Carotenoid Protein: Part I. Small-Angle Scattering, The journal of physical chemistry, 123 (2019) 9525-9535.

[61] A. Thurotte, R. Lopez-Igual, A. Wilson, L. Comolet, C. Bourcier de Carbon, F. Xiao, D. Kirilovsky, Regulation of Orange Carotenoid Protein Activity in Cyanobacterial Photoprotection, Plant Physiol, 169 (2015) 737-747.

[62] A. Wilson, M. Gwizdala, A. Mezzetti, M. Alexandre, C.A. Kerfeld, D. Kirilovsky, The essential role of the $\mathrm{N}$-terminal domain of the orange carotenoid protein in cyanobacterial photoprotection: importance of a positive charge for phycobilisome binding, Plant Cell, 24 (2012) 1972-1983.

[63] A. Wilson, F. Muzzopappa, D. Kirilovsky, Elucidation of the essential amino acids involved in the binding of the cyanobacterial Orange Carotenoid Protein to the phycobilisome, Biochimica et biophysica acta. Bioenergetics, 1863 (2022) 148504.

[64] W. Lou, D.M. Niedzwiedzki, R.J. Jiang, R.E. Blankenship, H. Liu, Binding of red form of Orange Carotenoid Protein (OCP) to phycobilisome is not sufficient for quenching, Biochimica et biophysica acta. Bioenergetics, 1861 (2020) 148155. 
Acknowledgments: We are grateful to Giorgio Schirò and Martin Weik for continued support of the project. We thank Sandrine Cot for technical assistance. We thank the ESRF and SLS synchrotron radiation facilities for beamtime allocation under long-term projects MX1992 and MX2329 (IBS BAG at ESRF), and acknowledge financial support by CEA, CNRS, Université Grenoble Alpes and Université Paris-Saclay. IBS acknowledges integration into the Interdisciplinary Research Institute of Grenoble (IRIG, CEA). This work was supported by the Agence Nationale de la Recherche (grants ANR-17-CE11-0018-01 to J.-P.C. and ANR-2018CE11-0005-02 to the three French laboratories), the Polish National Science Centre (NCN project 2018/31/N/ST4/03983), and used the platforms of the Grenoble Instruct-ERIC center (ISBG; UMS 3518 CNRS-CEA-UGA-EMBL) within the Grenoble Partnership for Structural Biology (PSB). Platform access was supported by FRISBI (ANR-10-INBS-05-02) and GRAL, a project of the University Grenoble Alpes graduate school (Écoles Universitaires de Recherche) CBH-EUR-GS (ANR-17-EURE-0003).

Competing interests: Authors declare no competing interests.

Data and materials availability: Atomic coordinates and structure factors have been deposited in the Protein Data Bank under the following accession codes: 7qd0, 7qcZ, 7qd1 and 7qd2. All other data are available in the main text or the supplementary materials. 


\section{Figures captions:}

Figure 1: Formation of $O C P^{R}$ is a multi-step reaction that spans 12 decades in time. The figure which presents a working model for OCP photoactivation, summarizes findings from multiple studies [12, 19, 23]. The wavelength of maximum difference-absorbance (after subtraction of the $\mathrm{OCP}^{\circ}$ signal) is indicated for all intermediate states characterized to date. The $\mathrm{P}_{1}, \mathrm{P}_{2}, \mathrm{P}_{2}$, and $\mathrm{P}_{3}$ were observed by Konold et al. 2019 in transient absorption UV-Vis

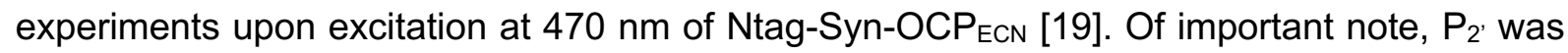
only observed in transient IR experiments, suggesting that it is characterized by a conformational change in the protein scaffold that does not influence the carotenoid electronic properties. $\mathrm{P}_{\mathrm{N}} / \mathrm{P}_{\mathrm{M}}$ and $\mathrm{P}_{\mathrm{x}}$ were observed by Maksimov et al. in time-resolved fluorimetry experiments upon excitation at $262 \mathrm{~nm}$ of the ECN-functionalized OCP-3FH mutant (W41F, W101F, W110F and W277H) featuring a single tryptophan at position W288 [23].

Figure 2: Photoactivation and recovery of native (non-tagged) CAN-functionalized Synechocystis and Planktothrix OCPs. (A and D) Absorbance spectra of Syn-OCP CAN (A) and Plankto-OCP $\mathrm{CAN}_{\mathrm{N}}(\mathrm{D})$ at different times of illumination. (B and E) Differential absorbance spectra derived from $A$ and $D$ respectively. ( $C$ and $F$ ) Differential absorbance spectra normalized at $470 \mathrm{~nm}$ derived from $A$ and D. (G-I) Kinetics of photoactivation (G) and recovery $(\mathrm{H}-\mathrm{I})$ of CAN-functionalized Syn-OCP (black) and Plankto-OCP (red). Inset in G: First $30 \mathrm{~s}$ of illumination. In (I), the effect of the presence of FRP is shown. The ratio FRP to OCP was 1:1. The accumulation of $O C P^{R}$ and its thermal deactivation were followed by increase and decrease of absorbance at $550 \mathrm{~nm}$. The OCPs were illuminated with white light (5 $000 \mu \mathrm{mol}$ photons $\mathrm{m}^{-2} . \mathrm{s}^{-1}$ ) at $9.5^{\circ} \mathrm{C}$. Error bars: standard deviation. Each curve represents the mean of three independent measurements, respectively.

Figure 3: Presence of a His-tag has an impact on photoactivation and recovery. Effect of presence and position of His-tag on photoactivation (A-B) and recovery (C-D) of CANfunctionalized Synechocystis and Planktothrix OCPs. The accumulation of OCPR and its thermal deactivation were followed by increase and decrease of absorbance at $550 \mathrm{~nm}$. The OCPs were illuminated with white light $\left(5000 \mu \mathrm{mol}\right.$ photons $\left.\mathrm{m}^{-2} . \mathrm{s}^{-1}\right)$ at $9.5^{\circ} \mathrm{C}$. Error bars: standard deviation. Each curve represents the mean of three independent measurements.

Figure 4: Effect of the nature of the functionalizing ketocarotenoid on photoactivation and recovery of N-terminus His-tagged Synechocystis and Planktothrix OCPs. (A-B) Kinetics of photoactivation of Syn-OCPs (A) and Plankto-OCPs (B) functionalized with ECN (red) or CAN (black). Inset in A and B: First $30 \mathrm{~s}$ of illumination. The OCPs were illuminated with white light $\left(5000 \mu \mathrm{mol}\right.$ photons $\left.\mathrm{m}^{-2} . \mathrm{s}^{-1}\right)$ at $9.5^{\circ} \mathrm{C}$. (C and D) Thermal recovery of $O C P^{R}$ in darkness of Syn-OCPs (C) and Plankto-OCPs (D) functionalized with ECN (red) or CAN (black). The accumulation of $O C P^{R}$ and its thermal recovery were followed by increase and decrease of absorbance at $550 \mathrm{n}$, respectively. Error bars: standard deviation. Each curve represents the mean of three independent measurements, respectively ( $E$ and $H$ ) Absorbance spectra of ECN-Syn $(E)$ and ECN-Plankto OCPs $(H)$ at different times of illumination. ( $F$ and $I)$ Raw difference absorbance spectra derived from $E$ and $H$ respectively. ( $G$ and $J$ ) Difference absorbance spectra derived from $E$ and $H$, respectively, after normalization on the $470 \mathrm{~nm}$ band.

Figure 5: Femtosecond transient absorption data collected on the Ntag-Plankto-OCP. (A, B) Transient absorption spectra measured after excitation at $532 \mathrm{~nm}$ are shown for time 
delays ranging between 0.14 and $1 \mathrm{~ns}$ for $(\mathrm{A})$ Ntag-Plankto-OCP $\mathrm{ECN}_{\mathrm{E}}$ and (B) Ntag-Plankto$\mathrm{OCP}_{\mathrm{CAN}}$. All datasets were normalized to -1 at bleaching minimum, in both spectral and temporal dimensions. (C, D) Decay Associated Spectra (DAS) obtained from the global fit of the transient absorption data spectra shown in (A) and (B), respectively. Data were fitted using four exponential components convolved by a Gaussian pulse (IRF, 110 fs FWHM) and an offset (long-lived photo-product > $10 \mathrm{~ns}$ ).

Figure 6: Nanosecond-second dynamics in CAN- and ECN-functionalized Plankto-OCP and Syn-OCP. Time evolution (50 ns to $1 \mathrm{~s}$ ) of the difference absorption signals at $550 \mathrm{~nm}$ recorded on Plankto-OCP $\mathrm{ECN}_{\text {(yellow), Plankto-OCP }}$ (red), Syn-OCP $\mathrm{ECN}_{\mathrm{CN}}$ (green) and Syn$\mathrm{OCP}_{\text {CAN }}$ (blue) after excitation by a $532 \mathrm{~nm}$ ns-pulse. The intermediate states proposed [12, $19,23]$ to underlie the observed absorption changes are indicated at the top of the figure. Measurements were carried out at $22^{\circ}$.

Figure 7: Effect of ketocarotenoid on OCP-PBS interaction. (A, C) Synechocystis (A) and Planktothrix (C) PBS fluorescence quenching induced at $23^{\circ} \mathrm{C}$ in $0.5 \mathrm{M}$ phosphate buffer by CAN (black) and ECN (red) functionalized Synechocystis (A) and Planktothrix (C) native (nontagged) OCPs. The OCP was pre-photoactivated by illumination with a strong white light (5 $000 \mu \mathrm{mol}$ photons $\mathrm{m}^{-2} . \mathrm{s}^{-1}$ ) at $4^{\circ} \mathrm{C}$. The ratio OCP:PBS was 40:1. (B, D) Dark recovery of fluorescence in Synechocystis (B) and Planktothrix (D) phycobilisomes. $100 \%$ of fluorescence in all graphs is the initial fluorescence of phycobilisomes without quenching. Error bars: standard deviation. Each curve represents the mean of three independent measurements.

Figure 8: Effect of the presence and position of a His-tag on OCP-PBS interaction. (A-C) Synechocystis (A, C) and Planktothrix (B) PBS fluorescence quenching induced at $23^{\circ} \mathrm{C}$ in 0.5 $\mathrm{M}$ phosphate buffer by native (non-tagged; black), N-tagged (red) and C- tagged (blue) CANfunctionalized Synechocystis (A) and Planktothrix (B, C) OCPs. The OCP was prephotoactivated by illumination of strong white light $\left(5000 \mu \mathrm{mol}\right.$ photons $\left.\mathrm{m}^{-2} \cdot \mathrm{s}^{-1}\right)$ at $4^{\circ} \mathrm{C}$. The ratio OCP:PBS was 40:1. (D-F) Dark recovery of fluorescence in Synechocystis (D, F) and Planktothrix (E) PBS. $100 \%$ of fluorescence in all graphs is the initial fluorescence of phycobilisomes without quenching. Error bars: standard deviation. Each curve represents the mean of three independent measurements.

Figure 9. The naturally occurring OCP dimer is found in all OCP crystals, including those of Plankto-OCP. Residues involved in the dimerization are highlighted in yellow, whereas residues involved in the formation of interface $X$ are highlighted in purple. Dark and light colouring indicate residues involved in polar and van der Walls interactions, respectively. Squares highlight residues present in alternate conformations in at least $50 \%$ of the analyzed Plankto-OCP chains. (A) The secondary structure of OCP is overall well conserved amongst Plankto-OCP structures obtained in different space groups and with different pigments. White dots highlight residues which are observed in alternate conformations. Blue stars indicate residues which have been shown to play a role in the OCP-PBS interaction, and red stars point to residues that could be at the origin of the stronger attachment of Plankto-OCP to the SynPBS. (B-C). The asymmetric unit of the $P 2_{1}$ crystals features a dimer (here shown as a ribbon with the two facing monomers colored sequence-wise, from cold ( $\mathrm{N}$-ter) to hot ( $\mathrm{C}$-ter) colors), whereas in $\mathrm{C} 2$ crystals, the dimer is crystallographic, hence only a single monomer is found in the asymmetric unit. (D) Polar contacts at the dimerization interface involve a conserved saltbridge between D19 and R27, as well as conserved H-bonds between facing D6 and T90, and 
between facing $\mathrm{N} 134$ and $\mathrm{N} 14, \mathrm{~T} 15$, and $\mathrm{T} 17 .(\mathrm{E}, \mathrm{F}, \mathrm{G})$ The $\mathrm{C} 2$ structures display a more compact conformation than the $P 2{ }_{1}$ structures, at both the dimer $(\mathrm{E}, \mathrm{F})$ and at the monomer levels $(G)$. The figure illustrates the trajectory followed by Plankto-OCP as we interpolate from the $C 2-C A N$ to the $P 2{ }_{1}$-CAN structure, highlighting the secondary structure elements which diverge most upon packing in the two crystal types. Arrows show the overall direction travelled by domains as we interpolate between the $\mathrm{C} 2$-Can and $P 2_{1}$-CAN Plankto-OCP.

Figure 10: The Planktothrix OCP features two similarly large packing interfaces in crystals. In Plankto-OCP crystals, a large interface, additional to the dimerization interface (Table S1), is found which we coined interface $X$ (Table S3). This interface, mainly contributed by by helices $\alpha \mathrm{D}, \alpha \mathrm{G}$ and $\alpha \mathrm{J}$ and by the $\alpha \mathrm{C}-\beta 4$ and $\beta 5-\beta 6$ loops, matches the dimerization interface, in $P 2{ }_{1}$ crystals (BSA of $\sim 1050 \AA^{2}$ ), but largely exceeds it, in the C2 crystals (BSA of $\sim 1600 \AA^{2}$ ). Changes in the extent of interface $X$ result in a reorientation of domains in each monomer forming the naturally-occurring dimer, and in an increase in the opening angle between monomers, in the dimer. Arrows indicate the direction and distance along which $\mathrm{Ca}$ atoms travel as we interpolate from the $P 2_{1}$ to the $C 2$ crystals.

Figure 11: Plankto-OCP structures differ in the compaction of the monomer as well as in the internal structure of the NTD. (A) The $C 2$ (orange), and $P 2_{1}$ chain $A$ (slate) and chain $B$ (grey) are overlaid as ribbons. Structural alignment, performed using the CTD atoms, highlight the change in opening angle between the CTD and the NTD. Indeed, the CTD structure is highly conserved with hardly no conformational changes observed amongst structures. Large differences are yet seen in the NTD, notably in the $\alpha \mathrm{C}-\alpha \mathrm{D}$ and $\alpha \mathrm{G}-\alpha \mathrm{H}$ loops, but as well in the relative positioning of $\alpha \mathrm{C}$ and $\mathrm{aD}$ helices. Here, the Plankto-OCP-CAN structures are shown, but the same observations were made when comparing the PlanktoOCP-ECN structures. (B) Close up view on CAN and on the residues lining the carotenoid tunnel. A change in the orientation of the carotenoid is seen upon compaction of the OCP structure due to change in space group.

Figure 12: Conformational changes observed upon change of the functionalizing carotenoid are limited to the NTD. (A) The structure of Plankto-OCP-CAN is shown as a ribbon colored from cold to hot colors as a function of the RMSD to the Plankto-OCP-ECN structure. (B) Close-up view on the carotenoid and residues lining the homonymous tunnel. The orientation in $(B)$ is similar to that in the upper panel of $(A)$.

Figure 13: Crystal packing traps different conformations of the Ntag-Plankto-OCP monomers. (A) Changes in Ca-Ca distances across in the various ECN- and CANfunctionalized $C 2$ and $P 2_{1}$ chains were monitored by computing Ca-Ca difference-distance matrices (DDM). As a reference structure, we used the C2 Plankto-OCP-CAN (2 first rows), or either chain $\mathrm{A}$ or $\mathrm{C}$ of the $\mathrm{P} 2{ }_{1}$ PlanktoOCP-CAN structure (third line). In each DDM, the lower and upper panels (separated by a sketch of the secondary structure) show the changes in CaCa distances for alternate conformers A and B with respect to the reference structure. Indeed, alternate conformations are seen in all Plankto-OCP structures. The overall similarity between upper and lower panels indicates that alternate conformations hardly affect the protein backbone. In the $P 2{ }_{1}$ crystals, two chains are found, which either display a more expanded (chain A) or a more compact (chain B) structure, due to changes in the opening angle between the NTD and the CTD. The DDM further indicates that these changes stem from helix $\alpha \mathrm{D}$, and the $\alpha \mathrm{C}-\alpha \mathrm{D}$ and $\alpha \mathrm{G}-\alpha \mathrm{H}$ loops, either drawing away (chain $\mathrm{A}$ ) or coming closer (chain $\mathrm{B}$ ) to the 
CTD, respectively. Comparison of the C2-CAN and C2-ECN structures suggests that the presence of CAN results in a more compact protein. Support to this hypothesis could not be obtained from comparison of the $P 2_{1}$ chain $A$ or $P 2_{1}$ chain $B$ structures. Nonetheless, we observe a similar trend when comparing, either in the CAN-functionalized or the ECNfunctionalized states, $P 2_{1}$ chain $C$ and $P 2_{1}$ chain $A$. Thus, changes in functionalizing carotenoid have a lesser influence on the OCP conformation than changes in space group. (B) DDM calculated for the isolated NTD of Syn-OCP-CAN vs. Syn-OCP-CAN. This DDM suggests that upon photoactivation, large scale conformational changes occur in the NTD that mainly result in helix $\alpha B$ drawing away from helices $\alpha D$ to $\alpha G$, while the $\alpha C-\alpha D$ loop comes closer to these and to $\alpha \mathrm{H}$ to $\alpha \mathrm{l}$. Also, $\alpha \mathrm{G}$ edges closer to helices $\alpha \mathrm{H}$ to $\alpha \mathrm{l}$, while the $\alpha \mathrm{G}-\alpha \mathrm{H}$ loop draws farther.

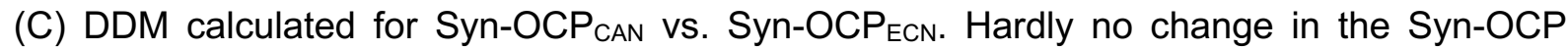
structure is seen upon change in the functionalizing carotenoid.

\section{Tables:}

Table 1: Lifetime, time constants and quantum yields obtained from femtosecond and nanosecond transient absorption experiments. (A) Lifetimes of ICT/S $1 / S^{*} / P_{1}$ states derived from femtosecond transient absorption measurements (from data in Figure 5 and Figure S2), and estimated formation quantum yields derived from preexponential factors at $490 \mathrm{~nm}$ (the magnitude of the bleaching band of each DAS was extracted from the fitting procedure; for more details, see the Materials and Methods section and Nizinski et al., submitted; doi.org/10.1101/2021.12.26.474187). The standard error is $10 \%$. $\mathrm{P}_{1}$ yields are corrected for its positive absorbance contribution at $500 \mathrm{~nm}$. (B) Time constants and yields (percentages in brackets) derived from ns-s transient absorption measurements for $P_{1}, P_{2} / P_{2}$ and $P_{3} / P_{N-M-X}$. Only in the case of $\mathrm{P}_{1}$ are the value in brackets actual quantum yields; for $\mathrm{P}_{2} / \mathrm{P}_{2}$, and $\mathrm{P}_{3} / \mathrm{P}_{\mathrm{N}-\mathrm{M} \text { - }}$ $x$, they rather indicate the relative contribution of the corresponding exponential terms to the overall decay and growing components (indicated in bold). Percentages in the OCPR column are formation quantum yields of the red absorbing intermediates remaining at $10 \mathrm{~ms}$ (determined by a comparative actinometry method with ruthenium complex; for details, see Material and Methods section and [45]).

Table 2. Data collection and refinement statistics. Statistics for the highest-resolution shell are shown in parentheses. 